\title{
Determination of Pesticides and Pesticide Degradates in Filtered Water by Direct Aqueous-Injection Liquid Chromatography-Tandem Mass Spectrometry
}

Chapter 11 of

Section B, Methods of the National Water Quality Laboratory

Book 5, Laboratory Analysis

Techniques and Methods 5-B11 



\section{Determination of Pesticides and Pesticide Degradates in Filtered Water by Direct Aqueous-Injection Liquid Chromatography- Tandem Mass Spectrometry}

By Mark W. Sandstrom, Leslie K. Kanagy, Cyrissa A. Anderson, and

Christopher J. Kanagy

Chapter 11 of

Section B, Methods of the National Water Quality Laboratory

Book 5, Laboratory Analysis

Techniques and Methods 5-B11 


\title{
U.S. Department of the Interior SALLY JEWELL, Secretary
}

\section{U.S. Geological Survey Suzette M. Kimball, Director}

\author{
U.S. Geological Survey, Reston, Virginia: 2015
}

For more information on the USGS - the Federal source for science about the Earth, its natural and living resources, natural hazards, and the environment—visit http://www.usgs.gov or call 1-888-ASK-USGS.

For an overview of USGS information products, including maps, imagery, and publications, visit http://www.usgs.gov/pubprod/.

Any use of trade, firm, or product names is for descriptive purposes only and does not imply endorsement by the U.S. Government.

Although this information product, for the most part, is in the public domain, it also may contain copyrighted materials as noted in the text. Permission to reproduce copyrighted items must be secured from the copyright owner.

Suggested citation:

Sandstrom, M.W., Kanagy, L.K., Anderson, C.A., Kanagy, C.J., 2015, Determination of pesticides and pesticide degradates in filtered water by direct aqueous-injection liquid chromatography-tandem mass spectrometry: U.S. Geological Survey Techniques and Methods, book 5, chap. B11, 54 p., http://dx.doi.org/10.3133/tm5B11.

ISSN 2328-7055 (online) 


\section{Contents}

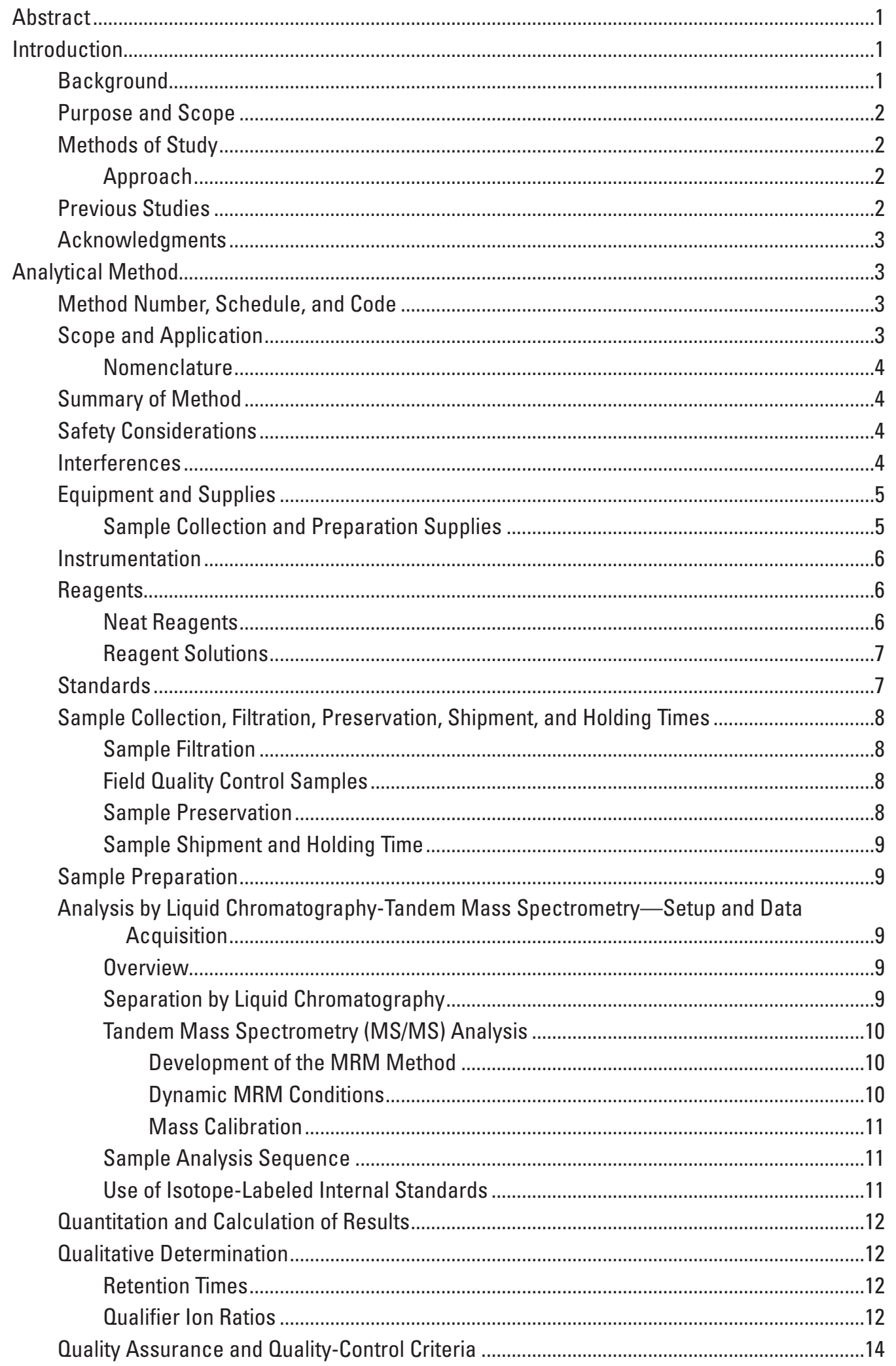


Routine laboratory OC Samples ..................................................................................14

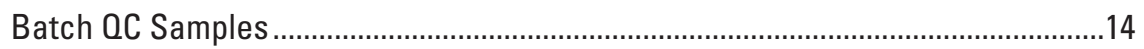

Instrument Laboratory QC Samples.........................................................................17

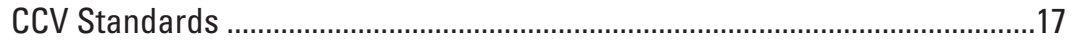

Instrument Detection Level Standards ............................................................17

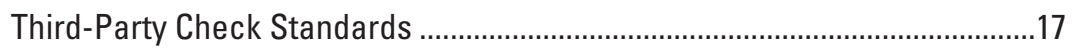

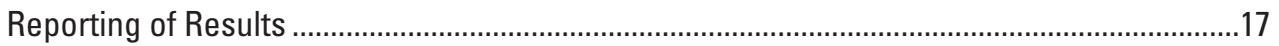

Results and Discussion of Method Validation Data .......................................................................18

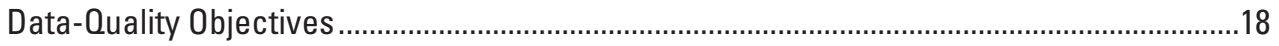

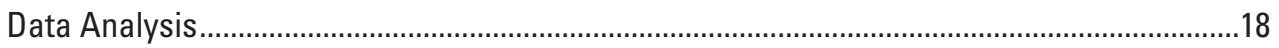

Assessment of Suitability for the DAI LC-MS/MS Method ................................................18

Analyte Response as a Function of Concentration ............................................................19

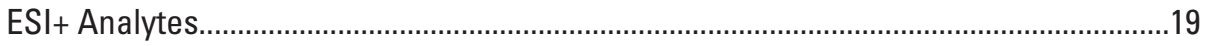

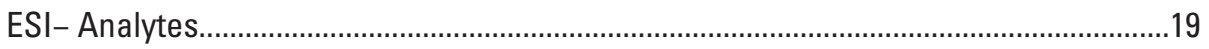

Method Detection Level Determination .............................................................................

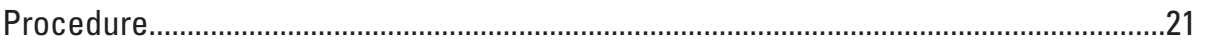

Method Detection Level Study Design...........................................................................21

Results of Method Detection Level Determination .........................................................21

Bias and Variability from Matrix-Spike Recovery Tests ........................................................22

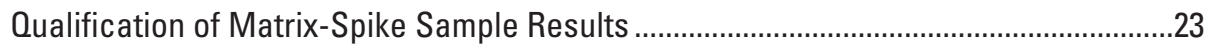

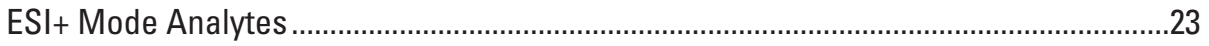

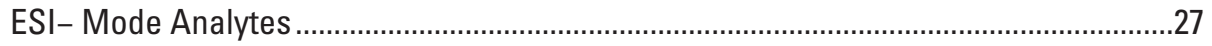

Performance of Laboratory Quality Control Samples During Implementation of Method .....27

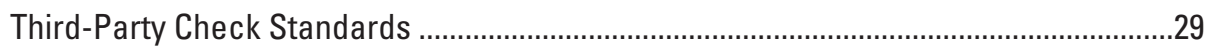

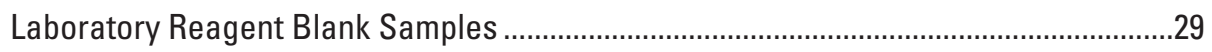

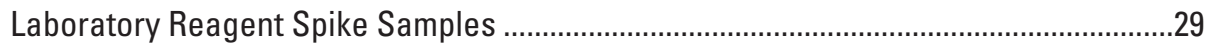

Application to Environmental Samples_-Paired-Sample Stream Study................................30

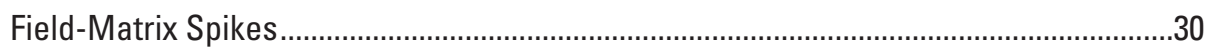

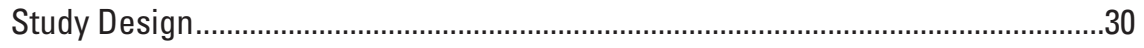

Spike Recovery in Stream Samples.......................................................................30

Relative Percent Difference of Spike Recovery .....................................................35

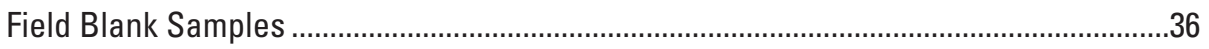

Environmental Sample Results ................................................................................36

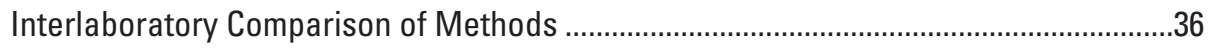

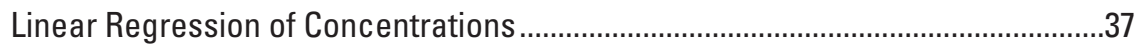

Detection Frequency in Stream Samples .............................................................37

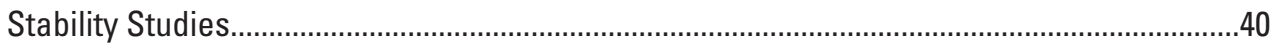

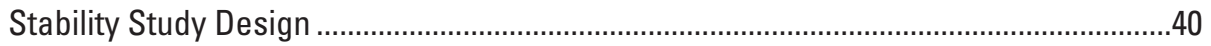

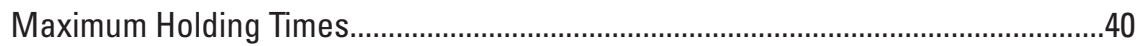

Stability Study Results ...........................................................................................

Tolerable Range of Variation................................................................................

Stability at $4{ }^{\circ} \mathrm{C}$ Pesticides ESI+ Mode Analytes.......................................................4

Stability at $4{ }^{\circ} \mathrm{C}$ Pesticides ESI- Mode Analytes.....................................................42

Stability at $4{ }^{\circ} \mathrm{C}$ Degradates ESI+ Mode Analytes................................................42

Stability at $4{ }^{\circ} \mathrm{C}$ Degradates ESI- Mode Analytes..................................................42

Stability at $-10^{\circ} \mathrm{C}$ Pesticides ESI+ Mode Analytes ................................................42 
Stability at $-10^{\circ} \mathrm{C}$ Pesticides ESI- Mode Analytes ................................................43

Stability at $-10^{\circ} \mathrm{C}$ Degradates ESI+ Mode Analytes ................................................43

Stability at $-10^{\circ} \mathrm{C}$ Degradates ESI- Mode Analytes ...............................................43

Pesticide Stability Using Dechlorination Reagent (Ascorbic Acid) ESI+ Mode Analytes ..................................................................................................

Pesticide Stability Using Dechlorination Reagent (Ascorbic Acid) ESI- Mode Analytes

Pesticide Degradate Stability Using Dechlorination Reagent (Ascorbic Acid) ESI+ Mode Analytes

Pesticide Degradate Stability Using Dechlorination Reagent (Ascorbic Acid) ESI- Mode Analytes

Pesticide Stability Using Antimicrobial Reagent (Citric Acid) ESI+ Mode Analytes

Pesticide Stability Using Antimicrobial Reagent (Citric Acid) ESI- Mode Analytes

Pesticide Degradate Stability Using Antimicrobial Reagent (Citric Acid) ESI+ Mode Analytes

Pesticide Degradate Stability Using Antimicrobial Reagent (Citric Acid) ESI- Mode Analytes .................................................................................. 45

Summary and Recommendations Based on Stability Study Results .......................45

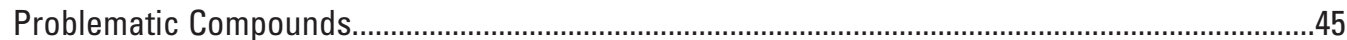

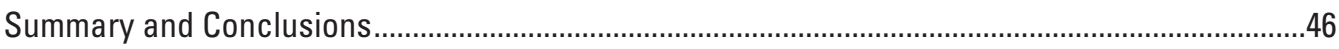

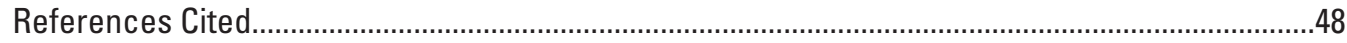

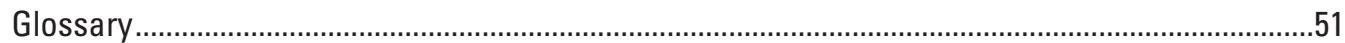

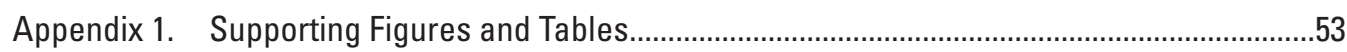

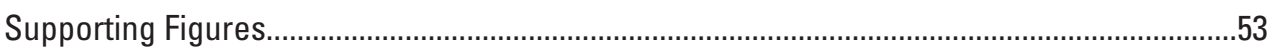

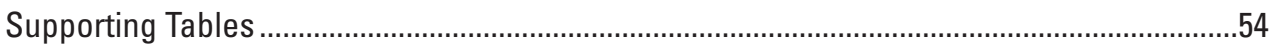

\section{Figures}

1. Example of a calibration curve for metolachlor determined by direct aqueous-injection liquid chromatography-tandem mass spectrometry showing response of metolachlor relative to the internal standard metolachlor-d6 plotted as a function of concentration, an expanded view of the lowest section of the calibration curve, and low concentration standard of metolachlor showing qualifier and quantifier multiple reaction monitoring ions for metolachlor...

2. The qualifier ion response ratio of atrazine and three atrazine degradates determined by direct aqueous-injection liquid chromatography-tandem mass spectrometry in positive electrospray ionization mode spiked at different concentrations in different matrices and reagent water.

3. Chromatograms of the multiple reaction monitoring ions for atrazine determined by direct aqueous-injection liquid chromatography-tandem mass spectrometry at different concentrations in four different matrices.

4. Response factors as a function of concentration for metolachlor and degradates determined by direct aqueous-injection liquid chromatography-tandem mass spectrometry in positive electrospray ionization mode 
5. Recovery of atrazine and degradates as a function of background environmental sample concentration determined by direct aqueous-injection liquid chromatography-tandem mass spectrometry in 146 field-matrix-spike samples collected during the National Water Quality Assessment Program field study from May to September 2012

6. Recovery of metolachlor determined by direct aqueous-injection liquid chromatography-tandem mass spectrometry in positive electrospray ionization mode in samples spiked at different concentrations in four different matrices.

7. Recovery of 2,4-D determined by direct aqueous-injection liquid chromatography-tandem mass spectrometry in negative electrospray ionization mode in samples spiked at different concentrations in four different sample matrices

8. Recovery of atrazine and degradates determined by direct aqueous-injection liquid chromatography-tandem mass spectrometry in 146 field-matrix-spike samples collected during the National Water Quality Assessment Program field study from May to September 2012

9. Comparison of selected pesticides determined by direct aqueous-injection liquid chromatography-tandem mass spectrometry gas chromatography-mass spectrometry in 150 samples from 48 stream sites collected during National Water Quality Assessment Program field comparison study from May to September 2012 .....39

10. Comparison of selected pesticides determined by direct aqueous-injection liquid chromatography-tandem mass spectrometry and liquid chromatography-mass spectrometry in 150 samples from 48 stream sites collected during National Water Quality Assessment Program field comparison study from May to September 2012 .....39

\section{Tables}

1. Information about pesticide compounds in direct aqueous-injection liquid chromatography-tandem mass spectrometry method. link

2. Concentrations of isotope-labeled pesticide compounds in internal standard solution link

3. High-performance liquid chromatography acquisition parameters link

4. Electrospray ionization positive mode pump gradient. link

5. Electrospray ionization negative mode pump gradient...... link

6. Optimum dynamic multiple reaction monitoring parameters for the determination of pesticide compounds by direct aqueous-injection liquid chromatographytandem mass spectrometry

7. Tandem mass spectrometry acquisition parameters link

8. Example of a sample sequence for determination of pesticide compounds in an analytical batch of 75 environmental samples and associated quality-control samples by direct aqueous-injection liquid chromatography-tandem mass spectrometry link

9. Assignment of pesticide compounds and surrogates to isotope-labeled internal standards for positive electrospray ionization and negative electrospray ionization mode analyses link

10. Acceptance criteria for retention times in direct aqueous-injection liquid chromatography-tandem mass spectrometry calculated from reproducibility of pesticide compound retention times in environmental water spike samples at concentrations from 10 to 5,075 nanograms per liter.... 
11. Acceptance criteria for relative ion intensity ratios for mass spectrometric techniques used for identification of organic contaminants in food products link

12. Acceptance criteria of qualifier ion ratios for analytes determined in positive electrospray ionization mode calculated from reagent water and matrix-spike samples link

13. Acceptance criteria of qualifier ion ratios for pesticide compounds determined in negative electrospray ionization mode calculated from reagent water and matrix-spike samples. link

14. Quality-control criteria for calibration standards, continuing calibration standards, and retention times for analyte identification from recent U.S. Environmental Protection Agency methods that use by direct aqueous-injection liquid chromatography-tandem mass spectrometry. link

15. Summary of response factor and parameters of linear fit of response factor by nominal concentration for positive electrospray ionization analytes. link

16. Summary of response factor and parameters of linear fit of response factor by nominal concentration for negative electrospray ionization mode analytes link

17. Summary of method detection levels, laboratory reporting levels, and low- and high-calibration standards for pesticide compounds determined by direct aqueous-injection liquid chromatography-tandem mass spectrometry.

18. Estimated method detection levels for pesticide compounds determined by direct aqueous-injection liquid chromatography-tandem mass spectrometry in positive electrospray ionization mode calculated according to U.S. Environmental Protection Agency procedure from analysis of seven replicate 5- and 10-nanograms per liter standards determined in seven consecutive analytical batches

19. Estimated method detection levels for pesticide compounds determined by direct aqueous-injection liquid chromatography-tandem mass spectrometry in positive electrospray ionization mode calculated according to U.S. Environmental Protection Agency procedure from analysis of eight replicate 5-, 10-, 25-, $50-$, and 100-nanograms per liter standards determined in one analytical batch. link

20. Estimated method detection levels for pesticide compounds determined by direct aqueous-injection liquid chromatography-tandem mass spectrometry in negative electrospray ionization mode according to U.S. Environmental Protection Agency procedure from replicate analysis of 5-, 10-, 50- and 100-nanograms per liter standards in seven consecutive analytical batches

21. Environmental samples collected for validation studies for direct aqueousinjection liquid chromatography-tandem mass spectrometry pesticide method......... link

22. Recovery of pesticide compounds in different water matrices and concentrations determined by direct aqueous-injection liquid chromatography-tandem mass spectrometry in positive electrospray ionization mode

23. Recovery of pesticide compounds in different water matrices and concentrations determined by direct aqueous-injection liquid chromatography-tandem mass spectrometry in negative electrospray ionization mode.

24. Recovery of analytes in third-party check standards analyzed by direct aqueous-injection liquid chromatography-tandem mass spectrometry in positive electrospray ionization mode from September 2012 to March 2013.

25. Recovery of analytes in third-party check standards analyzed by direct aqueous-injection liquid chromatography-tandem mass spectrometry in negative electrospray ionization mode from September 2012 to March 2013. link

26. Occurrence and concentrations of pesticide compounds in laboratory reagent blank samples analyzed by direct aqueous-injection liquid chromatographytandem mass spectrometry between June 19, 2012, and March 3, 2013. 
27. Recovery of pesticides determined by direct aqueous-injection liquid chromatography-tandem mass spectrometry in laboratory reagent-spike samples collected during implementation of the method from May 2012 to June 2013.............. link

28. Recovery of pesticides determined by direct aqueous-injection liquid chromatography-tandem mass spectrometry in 146 field-matrix-spike samples collected during National Water Quality Assessment Program field comparison study from May to September 2012 link

29. Recovery of pesticides determined by direct aqueous-injection liquid chromatography-tandem mass spectrometry in five field-matrix-spike samples fortified at 4,747 nanograms per liter collected during National Water Quality Assessment Program field comparison study from May to September 2012. link

30. Relative difference in recovery of pesticides determined by direct aqueous-injection liquid chromatography-tandem mass spectrometry in 50 field-matrix-spike and spike-replicate samples collected during National Water Quality Assessment Program field comparison study from May to September 2012 link

31. Summary of concentrations of pesticides determined by direct aqueous-injection liquid chromatography-tandem mass spectrometry in 43 field blank samples collected during National Water Quality Assessment Program field comparison study from May to September 2012

32. Summary of concentrations of pesticides determined by direct aqueous-injection liquid chromatography-tandem mass spectrometry in 150 stream-water samples collected during National Water Quality Assessment Program field comparison study from May to September 2012 link

33. Parameters for linear fit of concentrations determined by direct aqueous-injection liquid chromatography-tandem mass spectrometry and gas chromatography-mass spectrometry in environmental samples collected during National Water Quality Assessment Program field comparison study from May to September 2012 link

34. Parameters for linear fit of concentrations determined by direct aqueousinjection liquid chromatography-tandem mass spectrometry and liquid chromatography-mass spectrometry in environmental samples collected during National Water Quality Assessment Program field comparison study from May to September 2012

35. Consistency of detections or nondetections of pesticide compounds in 150 paired-stream samples determined by direct aqueous-injection liquid chromatography-tandem mass spectrometry and gas chromatography/mass spectrometry during National Water Quality Assessment Program field comparison study from May to September 2012

36. Sign test that number of detections in 150 paired-stream samples are the same for pesticide compounds analyzed by direct aqueous-injection liquid chromatography-tandem mass spectrometry and gas chromatography/mass spectrometry

37. Concentrations of pesticide compounds determined in National Water Quality Laboratory (NWOL) schedule 2033 gas chromatography/mass spectrometry in paired samples reported as nondetections in NWOL laboratory schedule 2437 direct aqueous-injection liquid chromatography-tandem mass spectrometry link

38. Concentrations of pesticide compounds determined in National Water Quality Laboratory (NWOL) schedule 2437 direct aqueous-injection liquid chromatography-tandem mass spectrometry in paired samples reported as nondetections in NWOL laboratory schedule 2033 gas chromatography/mass spectrometry 
39. Consistency of detections or nondetections of pesticide compounds in 150 paired-stream samples determined by direct aqueous-injection liquid chromatography-tandem mass spectrometry and liquid chromatography/mass spectrometry during National Water Quality Assessment Program field comparison study from May to September 2012.

40. Sign test that number of detections in 148 paired-stream samples are the same for pesticide compounds analyzed by direct aqueous-injection liquid chromatography-tandem mass spectrometry and liquid chromatography/mass spectrometry

41. Concentrations of pesticide compounds determined in National Water Quality Laboratory (NWOL) schedule 2060 liquid chromatography/mass spectrometry in paired samples reported as nondetections in NWQL laboratory schedule 2437 direct aqueous-injection liquid chromatography-tandem mass spectrometry

42. Concentrations of pesticide compounds determined in National Water Quality Laboratory (NWOL) schedule 2437 direct aqueous-injection liquid chromatography-tandem mass spectrometry in paired samples reported as nondetections in NWQL laboratory schedule 2060 liquid chromatography/mass spectrometry...

43. Stability (holding time) study design for pesticide compounds determined by direct aqueous-injection liquid chromatography-tandem mass spectrometry in laboratory reagent-water-spike samples

44. Summary of concentrations and recovery of 29 continuing calibration verification standards analyzed by direct aqueous-injection liquid chromatography-tandem mass spectrometry in positive electrospray ionization during stability studies to calculate tolerable range of variation and lower limit of recovery for holding-time determination link

45. Summary of concentrations and recovery of 29 continuing calibration verification standards analyzed by direct aqueous-injection liquid chromatography-tandem mass spectrometry in negative electrospray ionization during stability studies to calculate tolerable range of variation and lower limit of recovery for holding-time determination

46. Stability of pesticides in reagent water stored at 4 degrees Celsius for various times for as many as $\mathbf{2 8}$ days and analyzed by direct aqueous-injection liquid chromatography-tandem mass spectrometry in positive electrospray ionization mode....

47. Stability of pesticides in reagent water stored at 4 degrees Celsius for various times for as many as $\mathbf{2 8}$ days and analyzed by direct aqueous-injection liquid chromatography-tandem mass spectrometry in negative electrospray ionization mode.

48. Stability of degradates in reagent water stored at 4 degrees Celsius for various times for as many as $\mathbf{2 8}$ days and analyzed by direct aqueous-injection liquid chromatography-tandem mass spectrometry in positive electrospray ionization mode

49. Stability of degradates in reagent water stored at 4 degrees Celsius for various times for as many as $\mathbf{2 8}$ days and analyzed by direct aqueous-injection liquid chromatography-tandem mass spectrometry in negative electrospray ionization mode

50. Stability of pesticides in reagent water stored at -10 degrees Celsius for various times for as many as $\mathbf{2 8}$ days and analyzed by direct aqueous-injection liquid chromatography-tandem mass spectrometry in positive electrospray ionization mode...... 
51. Stability of pesticides in reagent water stored at -10 degrees Celsius for various times for as many as 28 days and analyzed by direct aqueous-injection liquid chromatography-tandem mass spectrometry in negative electrospray ionization mode link

52. Stability of degradates in reagent water stored at -10 degrees Celsius for various times for as many as 28 days and analyzed by direct aqueous-injection liquid chromatography-tandem mass spectrometry in positive electrospray ionization mode link

53. Stability of pesticide degradates in reagent water stored at -10 degrees Celsius for various times for as many as 28 days and analyzed by direct aqueous-injection liquid chromatography-tandem mass spectrometry in negative electrospray ionization mode link

54. Stability of pesticides in reagent water treated with ascorbic acid and stored at 4 degrees Celsius for various times for as many as 28 days and analyzed by direct aqueous-injection liquid chromatography-tandem mass spectrometry in positive electrospray ionization mode

55. Stability of pesticides in reagent water treated with dechlorination reagent (ascorbic acid) stored at 4 degrees Celsius for various times for as many as 28 days and analyzed by direct aqueous-injection liquid chromatography-tandem mass spectrometry in negative electrospray ionization mode.

56. Stability of degradates in reagent water treated with ascorbic acid and stored at 4 degrees Celsius for various times for as many as 28 days and analyzed by direct aqueous-injection liquid chromatography-tandem mass spectrometry in positive electrospray ionization mode

57. Stability of pesticide degradates in reagent water treated with dechlorination reagent (ascorbic acid) stored at 4 degrees Celsius for various times for as many as 28 days and analyzed by direct aqueous-injection liquid chromatographytandem mass spectrometry in negative electrospray ionization mode

58. Stability of pesticides in reagent water treated with citric acid pH 3.8 and stored at 4 degrees Celsius for various times for as many as 28 days and analyzed by direct aqueous-injection liquid chromatography-tandem mass spectrometry in positive electrospray ionization mode

59. Stability of pesticides in reagent water treated with citric acid pH 3.8 and stored at 4 degrees Celsius for various times for as many as 28 days and analyzed by direct aqueous-injection liquid chromatography-tandem mass spectrometry in negative electrospray ionization mode

60. Stability of degradates in reagent water treated with citric acid pH 3.8 and stored at 4 degrees Celsius for various times for as many as 28 days and analyzed by direct aqueous-injection liquid chromatography-tandem mass spectrometry in positive electrospray ionization mode

61. Stability of pesticide degradates in reagent water treated with citric acid pH 3.8 and stored at 4 degrees Celsius for various times for as many as 28 days and analyzed by direct aqueous-injection liquid chromatography-tandem mass spectrometry in negative electrospray ionization mode link

62. Summary of validation study results from selected tables link 


\section{Supporting Figures}

S1. The qualifier ion response ratio of pesticide compounds determined by LC-MS/MS in positive electrospray ionization mode fortified at different concentrations in different matrices in reagent water.

S2. The qualifier ion response ratio of pesticide compounds determined by LC-MS/MS in negative electrospray ionization mode fortified at different concentrations in different matrices and in reagent water.

S3. Graphs of response factor and parameters of linear fit of response factor by nominal concentration for direct aqueous-injection liquid chromatography-tandem mass spectrometry method positive electrospray ionization mode analytes

S4. Graphs of response factor and parameters of linear fit of response factor by nominal concentration for direct aqueous-injection liquid chromatography-tandem mass spectrometry method negative electrospray ionization mode analytes.

S5. Recovery of pesticide analytes determined by liquid chromatography-tandem mass spectrometry in positive electrospray ionization mode fortified at different concentrations in four different matrices.

S6. Recovery of pesticide analytes determined by liquid chromatography tandem mass spectrometry in negative electrospray ionization mode fortified at different concentrations in four different matrices.

S7. Recovery of pesticides determined by direct aqueous-injection liquid chromatography-tandem mass spectrometry in field-matrix-spike samples by site in 200 field-matrix-spike samples that were prepared for the National Water Quality Assessment Program field study from May to September 2012 link

S8. Pesticide compound recovery as a function of background environmental sample concentration determined by direct aqueous-injection liquid chromatography-tandem mass spectrometry in 146 field-matrix-spike samples that were prepared for the National Water Quality Assessment Program field study from May to September 2012

S9. Recovery of pesticides in reagent water after storage for as many as 28 days with different treatments determined by direct aqueous-injection liquid chromatography-tandem mass spectrometry in positive electrospray ionization mode

S10. Recovery of pesticide degradates in reagent water after storage for as many as 28 days with different treatments determined by direct aqueous-injection liquid chromatography-tandem mass spectrometry in positive electrospray ionization mode

S11. Pesticide degradates found in reagent water fortified with pesticides after storage for as many as 28 days with different treatments determined by direct aqueousinjection liquid chromatography-tandem mass spectrometry in positive electrospray ionization mode

S12. Recovery of pesticides in reagent water after storage for as many as 28 days with different treatments determined by direct aqueous-injection liquid chromatography-tandem mass spectrometry in negative electrospray ionization mode .

S13. Recovery of pesticide degradates in reagent water after storage for as many as 28 days with different treatments determined by direct aqueous-injection liquid chromatography-tandem mass spectrometry in negative electrospray ionization mode 
S14. Pesticide degradates found in reagent water fortified with pesticides after storage for as many as 28 days with different treatments determined by direct aqueous-injection liquid chromatography-tandem mass spectrometry in negative electrospray ionization mode link

\section{Supporting Tables}

S1. Groups of pesticide compounds used to prepare intermediate standard solutions of pesticide compounds for direct aqueous-injection liquid chromatographytandem mass spectrometry method....

S2. Preparation of primary dilution standard solutions in methanol used to make calibration standards for direct aqueous-injection liquid chromatography-tandem mass spectrometry method

S3. Examples of National Water Information System qualifier codes used by analyst with direct aqueous-injection liquid chromatography-tandem mass spectrometry method to qualify samples or analytes not meeting quality-control criteria.

S4. Summary of quality-control criteria for determination of pesticides by direct aqueous-injection liquid chromatography-tandem mass spectrometry.

S5. Pesticide compounds identified as high priority for method development but not included in direct aqueous-injection liquid chromatography-tandem mass spectrometry method for various reasons.

S6. Summary of response factor and parameters of linear fit of response factor by nominal concentration for direct aqueous-injection liquid chromatography-tandem mass spectrometry method positive electrospray ionization mode analytes. link

S7. Summary of response factor and parameters of linear fit of response factor by nominal concentration for direct aqueous-injection liquid chromatography-tandem mass spectrometry method negative electrospray ionization mode analytes............. link

S8. Recovery of pesticides determined by direct aqueous-injection liquid chromatography-tandem mass spectrometry method in field-matrix-spike samples collected during National Water Quality Assessment Program field comparison study from May to September 2012 excluded from data summaries because environmental concentration was greater than spike level.

S9. Summary of water quality parameters measured in streams when direct aqueous-injection liquid chromatography-tandem mass spectrometry method samples were collected during National Water Quality Assessment Program field comparison study from May to September 2012.

S10. Summary of ancillary water quality parameters for each stream site when direct aqueous-injection liquid chromatography-tandem mass spectrometry method samples were collected during National Water Quality Assessment Program field comparison study from May to September 2012.

S11. Degradates found in reagent water samples spiked with pesticides spiked at 250 nanograms per liter and stored at 4 degrees Celsius for as many as 28 days and analyzed by direct aqueous-injection liquid chromatography-tandem mass spectrometry in positive electrospray ionization and negative electrospray ionization modes.

S12. Degradates found in reagent water samples spiked with pesticides spiked at 250 nanograms per liter and stored at -10 degrees Celsius for as many as 133 days and analyzed by direct aqueous-injection liquid chromatography-tandem mass spectrometry in positive electrospray ionization and negative electrospray ionization modes. 


\section{Conversion Factors and Abbreviations}

SI to Inch/Pound

\begin{tabular}{lll}
\hline \multicolumn{1}{c}{ Multiply } & \multicolumn{1}{c}{ By } & \multicolumn{1}{c}{ To obtain } \\
\hline centimeter $(\mathrm{cm})$ & 0.3937 & inch (in.) \\
millimeter $(\mathrm{mm})$ & 0.03937 & inch (in.) \\
micrometer $(\mu \mathrm{m})$ & $0.3937 \times 10^{-6}$ & inch (in.) \\
\hline \multicolumn{3}{c}{ Volume } \\
\hline liter $(\mathrm{L})$ & 33.82 & ounce, fluid (fl. oz) \\
liter $(\mathrm{L})$ & 2.113 & pint (pt) \\
liter $(\mathrm{L})$ & 1.057 & quart (qt) \\
liter $(\mathrm{L})$ & 0.2642 & gallon (gal) \\
liter $(\mathrm{L})$ & 61.02 & cubic inch (in $\left.{ }^{3}\right)$ \\
microliter $(\mu \mathrm{L})$ & $0.3382 \times 10^{-4}$ & ounce, fluid (fl. oz) \\
milliliter $(\mathrm{mL})$ & 0.03382 & ounce, fluid (fl. oz) \\
\hline & \multicolumn{1}{c}{ Flow rate } & \\
\hline liter per minute $(\mathrm{L} / \mathrm{min})$ & 33.81 & fluid ounce per minute $(\mathrm{oz} / \mathrm{min})$ \\
milliliter per minute $(\mathrm{mL} / \mathrm{min})$ & 0.3381 & fluid ounce per minute $(\mathrm{oz} / \mathrm{min})$ \\
\hline & \multicolumn{2}{c}{ Mass } \\
\hline gram $(\mathrm{g})$ & 0.03527 & ounce, avoirdupois $(\mathrm{oz})$ \\
kilogram $(\mathrm{kg})$ & 2.205 & pound avoirdupois $(\mathrm{lb})$ \\
milligram $(\mathrm{mg})$ & $0.3527 \times 10^{-4}$ & ounce, avoirdupois $(\mathrm{oz})$ \\
\hline
\end{tabular}

Temperature in degrees Celsius $\left({ }^{\circ} \mathrm{C}\right)$ may be converted to degrees Fahrenheit $\left({ }^{\circ} \mathrm{F}\right)$ as follows:

${ }^{\circ} \mathrm{F}=\left(1.8 \mathrm{x}^{\circ} \mathrm{C}\right)+32$

Temperature in degrees Fahrenheit $\left({ }^{\circ} \mathrm{F}\right)$ may be converted to degrees Celsius $\left({ }^{\circ} \mathrm{C}\right)$ as follows:

${ }^{\circ} \mathrm{C}=\left({ }^{\circ} \mathrm{F}-32\right) / 1.8$

Specific conductance is given in microsiemens per centimeter at 25 degrees Celsius $(\mu \mathrm{S} / \mathrm{cm}$ at $\left.25^{\circ} \mathrm{C}\right)$.

Concentrations of chemical constituents in water are given in nanograms per liter (ng/L).

Concentration equivalent units (assuming 1 liter equals 1 kilogram):

ppm: $\mathrm{mg} / \mathrm{kg}, \mathrm{mg} / \mathrm{L}, \mu \mathrm{g} / \mathrm{mL}$

$\mathrm{ppb}: \quad \mu \mathrm{g} / \mathrm{L}, \mathrm{ng} / \mathrm{mL}$

ppt: $\quad \mathrm{ng} / \mathrm{L}, \mathrm{pg} / \mathrm{mL}$

NOTE TO USGS USERS: Use of liter (L) as a special name for cubic decimeter $\left(\mathrm{dm}^{3}\right)$ is restricted to the measurement of liquids and gases. No prefix other than milli should be used with liter. 


\section{Abbreviated Water-Quality Units}

$\begin{array}{ll}\mathrm{pg} & \text { picogram } \\ \mathrm{g} / \mathrm{L} & \text { gram per liter } \\ \mathrm{ng} / \mathrm{L} & \text { nanogram per liter } \\ \mathrm{ng} / \mathrm{mL} & \text { nanogram per milliliter } \\ \mathrm{v} / \mathrm{v} & \text { volume-to-volume } \\ \mu \mathrm{g} / \mathrm{mL} & \text { microgram per milliliter } \\ \mu \mathrm{g} / \mathrm{L} & \text { microgram per liter } \\ \mu \mathrm{L} & \text { microliter } \\ \mu \mathrm{m} & \text { micrometer } \\ \mu \mathrm{S} / \mathrm{cm} & \text { microsiemens per centimeter }\end{array}$

\section{Abbreviations}

ACS American Chemical Society

ASTM American Society for Testing and Materials

CAL calibration [standard]

CASRN Chemical Abstracts Service Registry Number

CCB continuing calibration blank

CCV continuing calibration verification [standard]

DAI direct aqueous injection

EPA U.S. Environmental Protection Agency

ESI electrospray ionization

ESI- negative electrospray ionization

ESI+ positive electrospray ionization

FEP fluorinated ethylene-propylene

GC-MS gas chromatography-mass spectrometry

HPLC high-performance liquid chromatography

IDL instrument detection level

IS internal standard

LIMS laboratory information management system

LC-MS liquid chromatography-mass spectrometry

LC-MS/MS liquid chromatography-tandem mass spectrometry

LRL laboratory reporting level

MDL method detection level 


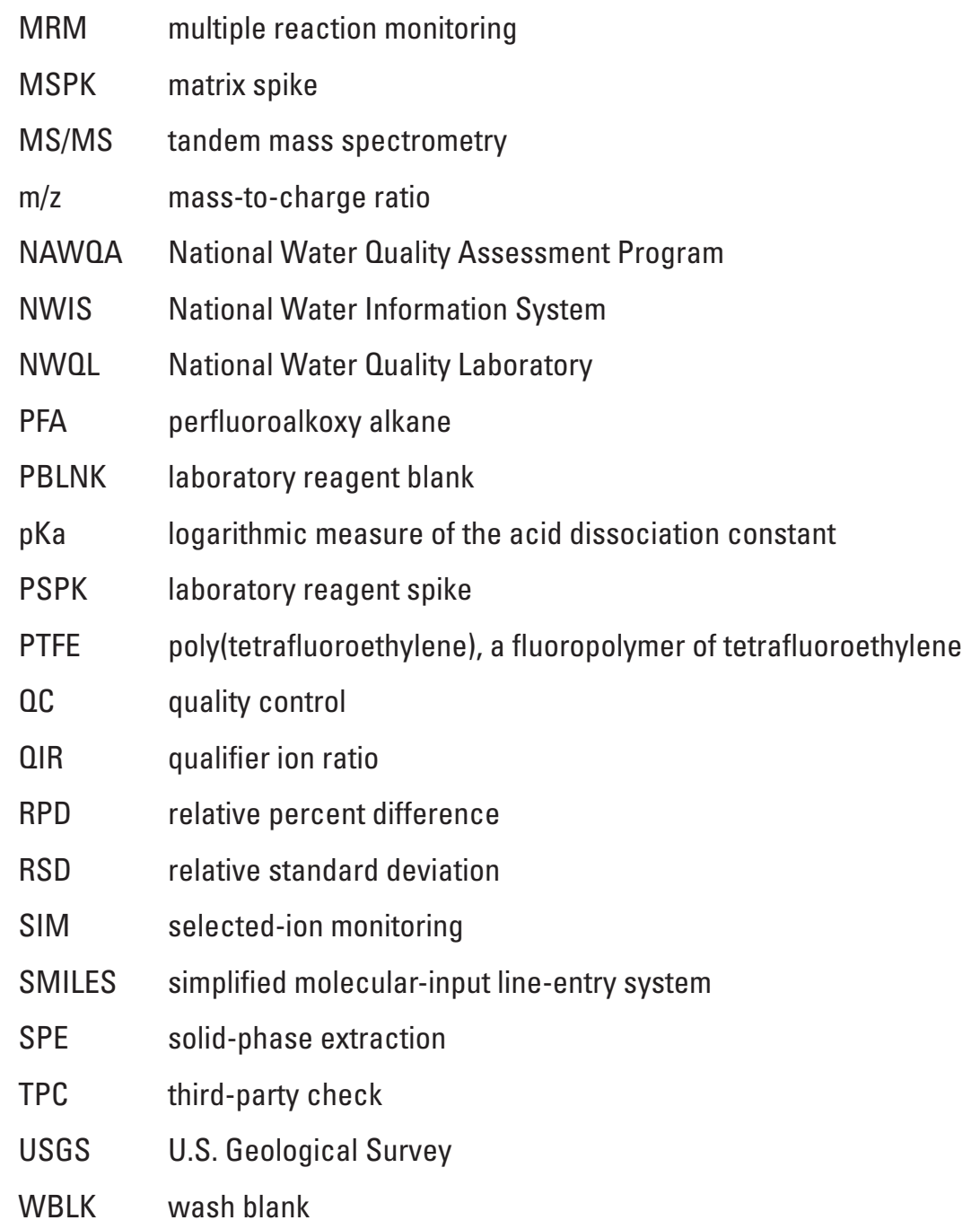




\title{
Determination of Pesticides and Pesticide Degradates in Filtered Water by Direct Aqueous-Injection Liquid Chromatography-Tandem Mass Spectrometry
}

\author{
By Mark W. Sandstrom, Leslie K. Kanagy, Cyrissa A. Anderson, and Christopher J. Kanagy
}

Abstract

A liquid chromatography-tandem mass spectrometry (LC-MS/MS) method was developed for determination of 229 pesticides compounds (113 pesticides and 116 pesticide degradates) in filtered water samples from stream and groundwater sites. The pesticides represent a broad range of chemical classes and were selected based on criteria such as current-use intensity, probability of occurrence in streams and groundwater, and toxicity to humans or aquatic organisms. More than half of the analytes are pesticide degradates. The method involves direct injection of a 100 -microliter $(\mu \mathrm{L})$ sample onto the LC-MS/MS without any sample preparation other than filtration. Samples are analyzed with two injections, one in electrospray ionization (ESI) positive mode and one in ESI negative mode, using dynamic multiple reaction monitoring (MRM) conditions, with two MRM transitions for each analyte. The LC-MS/MS instrument parameters were optimized for highest sensitivity for the most analytes. This report describes the analytical method and presents characteristics of the method validation including bias and variability, detection levels, and holding-time studies.

Mean recoveries of most analytes (223 of 229) were within data-quality objectives of $100 \pm 30$ percent at spike concentrations above method detection levels (MDLs) in all four matrices. The calculated MDLs ranged from 1 to 103 nanograms per liter (ng/L) for 182 analytes analyzed in the ESI positive mode, and from 2 to $106 \mathrm{ng} / \mathrm{L}$ for 42 analytes analyzed in the ESI negative mode. Five analytes had MDLs between 100 and $250 \mathrm{ng} / \mathrm{L}$. The stability studies in reagent water demonstrated that the largest number of the pesticide compounds (227 of 229) were stable after 14 days of storage at 4 degrees Celsius, so these were selected as the practical holding time and storage temperature for routine sample processing. The use of antimicrobial reagent citric acid to adjust the sample $\mathrm{pH}$ to about 4 also resulted in lower recoveries of some analytes, so it should not be used as a routine sample preservative.

\section{Introduction}

Analytical methods that are capable of determining a broad range of pesticide compounds (pesticide active ingredients and degradation products of these pesticides) at low concentrations are needed for monitoring water quality of the Nation's stream and groundwater resources.

\section{Background}

The U.S. Geological Survey (USGS) recently reevaluated the pesticides monitored in national studies and prioritized them based on a variety of factors including potential for occurrence and persistence in streams and groundwater and toxicity to humans and aquatic organisms (Norman and others, 2012). This prioritization resulted in a list of 257 pesticide compounds that were considered high priority (tier 1) for method development and future monitoring studies. The high-priority pesticide compounds included 78 new compounds not previously monitored in USGS analytical methods for determination of pesticides in water (based on their unique National Water Information System [NWIS] method code) as well as many pesticide compounds determined infrequently or in small research studies. In addition, 96 pesticide compounds are in commonly used USGS analytical methods (National Water Quality Laboratory [NWQL] laboratory schedules 2033 and 2060) that will be important for long-term monitoring studies.

Current NWQL analytical methods utilize solid-phase extraction (SPE) for sample concentration followed by both gas chromatography/mass spectrometry (GC-MS) (NWQL laboratory schedule 2033) and liquid chromatography/ mass spectrometry (LC-MS) (laboratory schedule 2060) for quantitation. Although these methods have suitable detection levels for environmental studies, comprehensive analysis of all high-priority pesticide compounds is expensive because multiple preparation steps and multiple analytical instruments are 
needed. In 2009, a new generation of liquid chromatographytandem mass spectrometry (LC-MS/MS) analytical instruments became commercially available that provided an alternative approach to determine a wide range of pesticide compounds (Greulich and Alder, 2008). The instruments had increased sensitivity and selectivity that allows for the determination of a broad range of analytes at environmentally relevant concentrations without any sample preparation.

Existing NWQL pesticide methods have several disadvantages such as bias and variability introduced by the extraction and evaporation procedures and the presence of interfering peaks due to the low selectivity of these detection techniques. Because of their high selectivity and sensitivity, tandem mass spectrometers can overcome these problems. In particular, high-performance liquid chromatographyelectrospray ionization-tandem mass spectrometry with dynamic multiple reaction monitoring (MRM) can be used without additional sample preparation.

\section{Purpose and Scope}

The purpose of the report is to describe the analytical procedure for a new direct aqueous-injection (DAI) LC-MS/MS method for determination of pesticide compounds in filtered water samples and provide characteristic performance measures for their validation in the method including bias and variability, method detection level (MDL), and stability studies. The bias and variability data were determined in four water types (reagent water, groundwater, and two different streams). MDLs were determined in reagent water using the U.S. Environmental Protection Agency (EPA) procedure (40 CFR part 142, appendix B). Stability studies were conducted in reagent water at two temperatures $\left(4^{\circ} \mathrm{C}\right.$ and $\left.-10{ }^{\circ} \mathrm{C}\right)$ for 28 days to determine sample holding-time protocols. The applicability of the method to a variety of stream samples and comparison with two other USGS methods for determination pesticides in water is described.

\section{Methods of Study}

\section{Approach}

The approach was to first obtain standards for as many of the high-priority ranked (tier 1) pesticide compounds as possible. There were 99 degradates ranked as high priority because they had been identified in previous USGS studies (Norman and others, 2012). In addition, any other degradates of these high-priority pesticides available from the EPA National Pesticide Standard Repository were also obtained. These degradates are available from the standards repository because they are included in the tolerance limits for each pesticide that may be found on foods based on potential risks to human health (40 CFR parts 136 and 180, respectively).

Although they are of concern for risk to human health in food, the degradates were not evaluated for likelihood to be present in streams and groundwater based on persistence, sorption, or toxicity to aquatic organisms. However, they were included in the method because their parent pesticide had a high usage rate, which is one of the factors used to prioritize degradates for monitoring studies (Sinclair and others, 2006). Pesticides were divided into 24 main analytical groups consisting of 1-31 pesticides per group. Analytical groups were based on pesticide use (herbicide, insecticide, fungicide) and chemical class (for example, triazine, acetanilide, and so forth).

The method was developed and validated using two identical Agilent 6460 LC-MS/MS instruments. One MS/MS instrument was operated in positive and one in negative electrospray ionization mode to obtain optimum performance for different groups of analytes. The first tasks were selection of precursor and product ions and optimization of MS/MS parameters (fragmentor voltage and collision energy). Analytes were optimized in the positive ionization mode first. If response was low (less than about $10^{3}$ area counts for integrated precursor ion) or previous literature indicated good performance in negative mode, analytes were optimized in negative ionization mode.

MS/MS instrument conditions and LC parameters were optimized for highest sensitivity of most analytes. Validation tests were conducted, including bias and variability in different matrices and range of concentrations, MDL, and stability studies (including assessment of preservation reagents).

\section{Previous Studies}

Methods based on GC-MS operated in the selected-ion monitoring (SIM) mode, combined with solid-phase extraction (SPE), have provided adequate sensitivity and selectivity needed for the determination of low concentrations of pesticide compounds in water samples. The GC-MS methods developed by the EPA for organic compounds in source water and water after treatment for drinking (Eichelberger and others, 1995) and by the USGS for studies of pesticides in streams and groundwater (Zaugg and others, 1995; Sandstrom and others, 2001) have been successfully used for more than two decades. However, a disadvantage of these GC-MS methods is that they are not suitable for polar or nonvolatile compounds, so it is difficult to have one method covering a wide range of pesticide compounds. In addition, because most degradates have a more polar character than the pesticide, LC-MS and LC-MS/MS are more suitable techniques for the determination of transformation products because the polar compounds are not amenable to volatilization needed for GC-MS without derivatization (Zwiener and Frimmel, 2004a).

The sensitivity of commercially available LC-MS/MS instruments priced for routine high-throughput use has improved over the last 5 years. Instrumental limits of quantitation of 1 picogram could be expected for electrospray ionization (ESI) in 2008 (Bester, 2008), and many mulitresidue methods for determination of pesticides by LC-MS/MS have recently been developed. A comparison of the scope, sensitivity, and selectivity of LC-MS/MS compared to GC-MS for more than 500 pesticide compounds showed better performance for LC-MS/MS for all except the organochlorine 
pesticides (Alder and others, 2006). Multiple reaction monitoring with a tandem mass spectrometer is more sensitive and selective technique compared to SIM in a single quadrupole mass spectrometer because multiple product ions from one or more precursor ions are measured rather than one ion (Le Grand and others, 2012). In addition, the effect of matrix interferences are reduced in MS/MS compared to MS because different molecules that share the same transition are more rarely found than molecules producing fragments of identical mass. As a consequence, peak identification, integration, and data processing are much easier and faster in LC-MS/MS and require less manual corrections compared to GC-MS (Lehotay and others, 2005).

The combination of the sensitivity of modern LC-MS/MS instruments and selectivity of the tandem mass spectrometry led to the development of direct injection methods that do not need the preconcentration steps of SPE (Backe and Field, 2012). A method using DAI of a 100 -micrograms per liter $(\mu \mathrm{L})$ sample LC-MS/MS in ESI negative mode (ESI-) was used to validate acetanilide degradates at the 50-nanogram per liter (ng/L) level in different water matrices (Fuhrman and Allan, 2003). Another study demonstrated that quantitative results for 300 pesticides in drinking water at $100 \mathrm{ng} / \mathrm{L}$ could be achieved by DAI LC-MS/MS with a $100-\mu \mathrm{L}$ sample injection without any sample enrichment or cleanup (Greulich and Alder, 2008). EPA method 538 uses DAI LC-MS/MS for determination of 11 pesticides in water at 11 to $1,500 \mathrm{ng} / \mathrm{L}$ (Shoemaker, 2009). Other applications of DAI LC-MS/MS methods for pesticides in water used two injections, one for ESI positive (ESI + ) analytes and one for ESI- analytes, including the determination of atrazine, simazine, propazine, and their respective dealkylated chlorotriazine metabolites; ametryn and prometryn and their respective dealkylated thiomethyltriazine metabolites; and S-metolachlor and its ethanesulfonic and oxanilic acid degradates at $50 \mathrm{ng} / \mathrm{L}$ (Huang and others, 2008); and 31 triazine, organophosphate, and acid pesticides at $15 \mathrm{ng} / \mathrm{L}$ (Díaz and others, 2008).

For MS/MS, at least two transition ions and the molecular ion are necessary to meet the identification criteria for an organic contaminant in food according to European Union guidelines (Zwiener and Frimmel, 2004b). These measures plus chromatographic retention-time specifications help avoid false positives in mass spectrometry, as demonstrated in the case of pesticides in water (Pozo and others, 2006) and steroid hormones in wastewater (Schlusener and Bester, 2005).

A dynamic (scheduled) MRM acquisition method monitors only the transitions within the target time window for each analyte, rather than a time segment of the chromatogram. For determination of a large number of pesticides in multi-residue LC-MS/MS analysis, dynamic MRM acquisition provides lower detection levels because the mass spectrometer spends more time with each MRM transition (increased dwell time, duty cycle, and thus data quality) without impacting on the cycle time, therefore assuring that enough data points are acquired (Fillâtre and others, 2010).

\section{Acknowledgments}

Alfred Lück (ETH, Switzerland) provided valuable assistance in the initial optimization of the LC-MS/MS parameters for pesticides. Robert Kent, Jerri Davis, and James Fallon kindly collected and filtered extra sample bottles for our validation studies.

\section{Analytical Method}

\section{Method Number, Schedule, and Code}

The analytical method and validation data described in this report were evaluated by the USGS Office of Water Quality and approved as USGS method O-2437-15 on June 1, 2015. Pesticide compounds are analyzed using DAI LC-MS/ MS for filtered water using NWQL schedule 2437 and laboratory method code LCM60. The laboratory method code is used in the USGS National Water Information System (NWIS) data system (http://nwis.waterdata.usgs.gov/nwis/qw) to describe methods used for determination of water-quality parameters. In addition, the NWIS water-quality software (QWDATA) uses a reference table of parameter-method code pairs (called analyteID) for reporting rounded values to data users (http:// help.waterdata.usgs.gov/codes-and-parameters/codes\#WQ).

\section{Scope and Application}

Method O-2437-15 is used to determine 229 pesticide compounds (113 pesticides and 116 pesticide degradates) in filtered stream and groundwater samples (table 1). The range of concentrations of calibration standards is from 1 to $10,000 \mathrm{ng} / \mathrm{L}$. The detection levels in reagent water range from 1 to $250 \mathrm{ng} / \mathrm{L}$, with a median of $3 \mathrm{ng} / \mathrm{L}$ for pesticide compounds determined in $\mathrm{ESI}+$ mode, and from 2 to $250 \mathrm{ng} / \mathrm{L}$, with a median of $40 \mathrm{ng} / \mathrm{L}$ for pesticide compounds determined in the ESI- mode.

Information about the pesticide compounds summarized in table 1 includes pesticide compound names, USGS parameter codes, analyte identifications numbers (IDs), MDLs, laboratory reporting level (LRL), and the lab code. Lab codes are used for grouping of analytes in the NWQL laboratory information management system (LIMS) system and are 9030 for the ESI+ analytes, 9031 for the ESI- analytes, and 3406 for the sample volume. Other information about the pesticides includes the Chemical Abstracts Service Registry Number $\left(\mathrm{CASRN}^{1}\right)$ if available, molecular weight, molecular formula, analytical use (pesticide, degradate, internal standard), and SMILES (simplified molecular-input line-entry system) notation. The SMILES is a specification in the form of a line

\footnotetext{
${ }^{1}$ This report contains CAS Registry Numbers ${ }^{\circledR}$, which is a registered trademark of the American Chemical Society. CAS recommends the verification of the CASRNs through CAS Client Services ${ }^{\mathrm{sin}}$.
} 
notation for describing the structure of molecules using short ASCII strings. SMILES strings can be imported by most molecule editors for conversion back into two-dimensional drawings or three-dimensional models of the molecules (Weininger, 1988; O'Boyle, 2012). The analytical method group, pesticide group, and validation quality code were used in this report for discussion of validation results and sorting of pesticide compounds in figures and tables. The pesticide group is the name of the active form of pesticide applied in the environment and provides a way to link pesticide degradates to the active form of pesticide that was used. The validation quality codes are unique to method validation qualification of data in this report and can be A, acceptable; E, estimated; or D, deleted from method in May 2013. Finally, information about whether the pesticide compound was new or in previous USGS methods based on their unique NWIS analyteID is shown.

The new method includes many high-priority (tier 1) pesticide compounds that were in previous USGS pesticide methods that will be useful for long-term trend analysis. For example, 37 pesticide compounds in the new method are in method O-1126-95, 38 in method O-2002-01, and 42 in method O-2060-01 determined at the NWQL, and 17 pesticide compounds in method O-2138-02 and 20 in method O-2139-03 determined at the Kansas Organic Geochemistry Research Laboratory (some pesticide compounds are in more than one previous method). Other high-priority (tier 1) analytes were not in any previous USGS methods, and represent new analytes for USGS studies. These include 22 pesticide active ingredients and 56 pesticide degradates (table 1).

\section{Nomenclature}

Terms used for the analytes in this report are "pesticide" for the active ingredient, "degradate" for the pesticide metabolite or degradate, or "pesticide compound" for both. Synonyms for these terms used in literature include parent for pesticide and daughter or transformation product for the degradate.

Common names for pesticide compounds in this report (table 1) generally are the same as those used in NWIS. Some alternatives to the NWIS names were selected for this report to make the names used for a chemical class more consistent or the text more readable. For example, the names of all organophosphate degradates used the "oxon" format rather than "oxygen analog." Similarly, the acetanilide degradate names all used the abbreviations SA for sulfonic acid, SAA for sulfinylacetyl acid, and OA for oxanilic acid. For the triazine degradates, deethylatrazine is used as a synonym for the atrazine degradate 2-chloro-4-isopropylamino-6-amino-s-triazine (CIAT), deisopropylatrazine is used as a synonym for the atrazine degradate 2-chloro4-ethylylamino-6-amino-s-triazine (CEAT), and didealkylatrazine is used as a synonym for the atrazine degradate 2-chloro-4,6-diamino-s-triazine (CAAT) in this report.

\section{Summary of Method}

Samples are filtered at the field site using a 0.7-micrometer $(\mu \mathrm{m})$ nominal glass fiber filter and shipped to the laboratory. Prior to analysis at the laboratory, a $900-\mu \mathrm{L}$ subsample is removed and placed in a 2-milliliter $(\mathrm{mL})$ analytical vial. A solution of isotope-labeled (enriched) pesticides is added to the samples for use as internal standards, and $90 \mu \mathrm{L}$ of methanol is added to modify the sample matrix for optimal chromatographic performance. Pesticides are determined in the samples in two groups, one by the MS/MS operated in positive electrospray ionization (ESI+) mode, the other in negative electrospray ionization (ESI-) mode. For each group an aliquot of the sample $(100 \mu \mathrm{L})$ is injected onto a liquid chromatographic column ( $\mathrm{C} 18$ phase), and the analytes are separated using binary mobile phase of formic acid/ammonium formate-methanol for ESI+ mode or acetic acid-acetonitrile for ESI- mode. A dynamic multiple reaction monitoring (MRM) method is used on the MS/MS to collect specific quantification and qualifier ions for each analyte. Analyte identification is based on agreement between known standards with respect to chromatographic retention time and the ratio of the two characteristic MRMs. The concentration of each identified component is determined using the internal standard technique.

\section{Safety Considerations}

All procedures that require the use of solvents, such as preparation of mobile phases and calibration standards, should be conducted in a fume hood. Eye protection, nitrile gloves, and protective clothing should be worn when handling reagents, solvents, or standards.

Liquid waste produced during sample preparation and analysis, including solvents (methanol, acetonitrile) used for rinsing glassware, unused mobile phase mixtures, and mobile phase eluting from the high-performance liquid chromatography (HPLC) instrument not transferred to the MS/MS instrument, is collected in thick-walled plastic carboys. Solid waste, including analytical vials containing samples, spike mixtures, and methanol, is stored in glass carboys. All liquid and solid waste is stored and disposed according to the NWQL waste handling procedures.

\section{Interferences}

All glassware must be thoroughly cleaned to prevent contamination and avoid interferences. All glassware is washed with detergent, rinsed with tap water, and finally rinsed with methanol. Nonvolumetric glassware such as sample containers, analytical vials, and micropipette bores and stainlesssteel spatulas must be either solvent-rinsed (with methanol) or baked in a furnace at $450{ }^{\circ} \mathrm{C}$ for 2 hours (h). Volumetric glassware must be solvent rinsed (with methanol followed by solvent used for standard). Clean glassware is covered with aluminum foil for storage. 
Disposable items made of high-purity polypropylene such as transfer pipette tips, syringes, and syringe-tip filters are suitable for this tandem mass spectrometry method. These items must be pre-rinsed with solvent or sample prior to use.

Method interferences may be caused by contaminants in solvents, reagents, disposable transfer pipets, gloves, and other laboratory supplies. These consumable items should be demonstrated to be free from interferences by routine analysis of laboratory reagent blanks. Identification of contaminants or interferences in MRM chromatograms will require topical blanks that examine only specific aspects of the method (solvents, vials) to identify and remove them.

Good laboratory practices must be followed at all times when preparing reagents and samples. Analysts and sample collectors should wear nitrile gloves for safety reasons and to avoid contamination of the samples. All reagent and sample preparation steps must be conducted in a clean environment, and care must be taken to prevent contamination while preparing samples and standards. Material that comes in contact with samples must only be made of glass, metal, ceramics, or fluoropolymers (for example, fluorinated ethylene-propylene [FEP]). Flexible plastic materials containing plasticizers such as pipette bulbs, polypropylene squeeze bottles, and Tygon tubing must be replaced with plasticizer-free material such as mechanical dispensers, FEP squeeze bottles, and FEP and perfluoroalkoxy alkane (PFA) tubing. Some high-purity plastic materials can be used if shown to be contamination free using this method, for example, disposable pipette tips, filters, and syringes constructed of high-purity polypropylene. All standard solution preparations are described in the standards laboratory notebook, with concentrations, lot numbers, and expiration dates listed in the notebook and on the vial label.

\section{Equipment and Supplies}

The equipment and supplies obtained from vendors listed below were tested during development of this method. Specific brand names and catalog numbers are shown for illustration, and equivalent vendors can be used where appropriate.

- Standard solution containers-Amber borosilicate glass vials fitted with polytetrafluoroethylene (PTFE)-lined screw cap, 20-mL (C\&G Containers Inc., Lafayette, La., container 109149)

- Analytical vials-Amber glass vials, 2-mL (National Scientific, Rockwood, Tenn., Target DP, C4000-2W), with 11-millimeter (mm)-diameter polypropylene screw caps lined with PTFE/red silicone septa (Agilent Technology, Wilmington, Del., catalog number [cat. no.] 5182-0717, PTFEsilicone-PTFE liner). These caps appear to minimize errors in the HPLC autosampler and stoppage of the analytical sequence.
- Analytical balance - Capable of weighing to nearest 0.01 milligram (Mettler Toledo, Columbus, Ohio, model XS205, or equivalent)

- Digital microdispenser-Various volume ranges including $10 \mu \mathrm{L}, 25 \mu \mathrm{L}$, and $100 \mu \mathrm{L}$ (VWR Scientific, Radnor, Pa., cat. no. 53506-121), with disposable glass bores. These are used for preparation of mixed compound intermediate standards and calibration standards.

- Digital pipette-Various volume ranges including $10 \mathrm{~mL}$ (Rainin Pipettes, Oakland, Calif., model EDPPlus $1000,100 \mu \mathrm{L}$ to $10,000 \mu \mathrm{L}$ ) and $20 \mathrm{~mL}$ (Rainin Pipettes, Oakland, Calif., model EDP-Plus 2000, $200 \mu \mathrm{L}$ to $20,000 \mu \mathrm{L}$ ) electronic pipettes, with disposable polypropylene tips. These pipettes are used to prepare mixed intermediate-calibration solutions and to transfer samples from collection containers to analytical vials. The pipette tips are rinsed once with clean solvent or the sample to pre-wet the tip and remove any contaminants.

- Transfer pipette-Manual macro pipette, variable volume, 0.5 to $5 \mathrm{~mL}$ for use with glass Pasteur pipettes (VWR International, Radnor, Pa., Socorex model 835.02PP, Wheaton part number W810316)

- Disposable glass pipets-Disposable Pasteur pipets, glass, 14.6-centimeter $(\mathrm{cm})$ long $\times$ 7-mm diameter (VWR International, Radnor, Pa., model 14672-200)

- Vortex mixer-Vortex Genie 2 (Scientific Industries, Bohemia, N.Y., model G560)

- Wash bottle-Nalgene wash bottles, Teflon FEP, Narrow Mouth, 250-mL (Thermo Scientific, Waltham, Mass.). These "squeeze" bottles are used to dispense acetonitrile, methanol, laboratory reagent water, and other solvents into glass beakers and flasks.

- Spatula - Rounded spatula/spoon tip, stainless steel, (VWR International, Radnor, Pa., model 82027-522). These spatulas are used to transfer neat standards to volumetric flasks.

- Automatic ampule sealer-Ampulmatic, model number 290001 (Bioscience Inc., Bethlehem, Pa.)

\section{Sample Collection and Preparation Supplies}

- Syringe-Disposable, high-purity polypropylene, 30-mL, male Luer-lock tip (Norm-Ject $30 \mathrm{~mL}$, polypropylene; no rubber, latex or silicone oil [VWR International, Radnor, Pa., model no 80076-426 or USGS OneStop number Q763FLD]). 
Determination of Pesticides and Pesticide Degradates in Filtered Water

- Syringe-tip filters-Disposable, 25-mm diameter, polypropylene housing with female Luer-lock inlet and male Luer outlet and glass fiber filter (Whatman $\mathrm{GF} / \mathrm{F}$ with graded multifiber [GMF], 0.7- $\mu \mathrm{m}$ nominal pore diameter, Whatman GD/X syringe [GE Healthcare Bio-Sciences, Pittsburgh, Pa., cat. no. 6902-2504 or USGS OneStop number Q762FLD]). The filter is specifically designed for high-particulate-loaded samples. Constructed of a pigment-free polypropylene housing with a prefiltration stack of Whatman GMF 150 (graded density) and GF/F glass microfiber media. GMF 150 and GF/F are produced from 100 -percent borosilicate-glass microfiber. The graded density GMF 150 medium has a coarse top layer meshed with a fine bottom layer that retains particles to $1.0 \mu \mathrm{m}$. A GF/F filter then retains particles down to $0.7 \mu \mathrm{m}$.

- Stainless-steel bore-disposable bore, 5.08-cm length, 18 -gauge with Luer fitting (USGS OneStop number Q764FLD).

- The syringe, syringe-tip filter, and stainless steel bore are available as a sampling kit (USGS OneStop number Q765FLD).

- Sample containers-Amber borosilicate-glass vials fitted with PTFE-lined screw cap, 40-mL (C\&G containers, Inc. [Lafayette, La., container number 107941] or 20-mL [C\&G Containers, Inc., container 109149]), pre-cleaned by vendor protocol B (volatile organic compounds, wash and rinse with American Society for Testing and Materials (ASTM) type 1 water [ASTM Standard D-1193-91, West Conshohocken, Pa.]). Vials are shipped in a foam sleeve that can be used for storage of vials in a freezer.

\section{Instrumentation}

- LC-MS/MS system-The samples were analyzed using an Agilent model 6460A triple quadrupole (MS/MS) mass spectrometer equipped with electrospray ionization with Agilent jet stream technology (Agilent Technology, Wilmington, Del.). The MS/MS was coupled to an Agilent model 1200 Series LC system. The LC system consisted of a binary pump (G1312B), vacuum solvent degasser (G1379B), automatic liquid sampler (G1367D), thermostated sample tray (G1330B), thermostated column compartment (G1316B), solvent degasser (G1379B), and MassHunter data acquisition system. The sampler included a high-volume kit (G1367-68713) for injection volumes greater than $40 \mu \mathrm{L}$. The thermostated column compartment allows column temperatures to as much as $100^{\circ} \mathrm{C}$ and pressures to as much as 600 bar. Narrow bore capillary tubing $(0.12$ - $\mathrm{mm}$ interior diameter) was used to connect the LC system components to maximize chromatographic peak height and resolution. Qualitative and quantitative data analyses were performed using the Agilent MassHunter data system (version B.04.00).

\section{Reagents}

\section{Neat Reagents}

- Nitrogen gas-High-purity nitrogen (99.5 percent pure), dry, supplied as much as 30 liters per minute $(\mathrm{L} / \mathrm{min})$, is used as a drying gas, sheath gas, nebulizing gas, and to pressurize the calibrant delivery system. A high-capacity gas conditioner attached to the gas inlet tubing of the LC-MS/MS is used to remove hydrocarbon contamination from the nitrogen source for collision cell reagent gas (Agilent Technology, Wilmington, Del., part no. BHT-4).

- Laboratory reagent water-High-purity organic-free reagent water was obtained by passing deionized water through a Solution 2000 ultrapure water purification system that contains charcoal prefilters and a dualwavelength ultraviolet oxidizer lamp for removal of trace organic compounds (Aqua Solutions Inc., Jasper, Ga., Model 2121AL)

- Solvents - acetonitrile, isopropanol, dimethylsulfoxide, and methanol (B\&J Brand, pesticide grade, Honeywell-Burdick and Jackson, Muskegon, Mich.)

- Ammonium formate $\left(\mathrm{NH}_{4} \mathrm{CHO} 2, \mathrm{CASRN} 540-69-2\right)-$ LC-MS Ultra grade (Fluka cat no. 14266, SigmaAldrich, St. Louis, Mo.)

- Ammonium acetate (NH4C2H3O2, CASRN 631-61-6) - LC-MS Ultra grade (Fluka, cat no. 14267, Sigma-Aldrich, St. Louis, Mo.)

- Formic acid-LC-MS Ultra grade (Fluka cat no 14265, Sigma-Aldrich, St. Louis, Mo.)

- Acetic acid-Eluent additive for LC-MS grade (Fluka cat no 49199, Sigma-Aldrich, St. Louis, Mo.)

- Citric acid monohydrate (C6H8O7 H2O, CASRN 5949-29-1)-American Chemical Society (ACS) reagent grade (cat. no. C-1909, Sigma-Aldrich, St. Louis, Mo.)

- Trisodium citrate dihydrate $(\mathrm{C} 6 \mathrm{H} 5 \mathrm{Na} 3 \mathrm{O} 72 \mathrm{H} 2 \mathrm{O}$, CASRN 6132-04-3) - ACS reagent grade (cat. no. ED-355, Sigma-Aldrich, St. Louis, Mo.)

- Ethylenediaminetetraacetic acid (EDTA) trisodium salt hydrate (C10H13N2Na3O8 H2O, CASRN 857156-02) - ACS reagent grade (cat. no. C-1909, SigmaAldrich, St. Louis, Mo.) 
- L-Ascorbic acid (CASRN 50-81-7) — High purity (cat. no. 255564, Sigma-Aldrich, St. Louis, Mo.)

- Electrospray calibrant mix - Pre-mixed solution of perfluorinated compounds that are distributed over the mass range (112 to 2,834 mass-to-charge ratio [m/z]) for tuning the electrospray MS/MS (Agilent Technology, Wilmington, Del., part no. G1969-85000 EI-L)

\section{Reagent Solutions}

- 1-molar (M) ammonium formate solution-Dissolve 65.69 grams $(\mathrm{g})$ of ammonium formate in 1 liter $(\mathrm{L})$ of laboratory reagent water.

- 1-M formic acid solution-Dilute $38.8 \mathrm{~mL}$ of 98-percent formic acid with laboratory reagent water to a final volume of $1 \mathrm{~L}$.

- ESI positive mode mobile phase A-Dilute $10 \mathrm{~mL}$ of 1-M ammonium formate solution and $12 \mathrm{~mL} \mathrm{1-M} \mathrm{for-}$ mic acid solution with lab laboratory reagent water to a final volume of $1 \mathrm{~L}$ ( 0.05 percent volume-to-volume $[\mathrm{v} / \mathrm{v}]$ ammonium formate $[0.010 \mathrm{M} ; \mathrm{pH} 6.3-7.3]$ and 0.05 percent $[\mathrm{v} / \mathrm{v}]$ formic acid $[0.012 \mathrm{M} ; \mathrm{pH}=1.88$; logarithmic measure of the acid dissociation constant $[\mathrm{pKa}]=3.75])$ in water.

- ESI negative mode mobile phase A-Dilute $2 \mathrm{~mL}$ of acetic acid with laboratory reagent water to a final volume of $1 \mathrm{~L}(0.2$ percent $[\mathrm{v} / \mathrm{v}]$ acetic acid in water; $0.0334 \mathrm{M} ; \mathrm{pH}=3.12 ; \mathrm{pKa}=4.76$ ).

\section{Standards}

Analytical reference standards were obtained primarily from the EPA National Pesticide Standards Repository (Ft. Meade, Md.). If standards were not available from the Standards Repository, they were obtained from EQ Laboratories (Athens, Ga.) or Sigma-Aldrich (St. Louis, Mo.). Isotopelabeled reference standards were obtained from Cambridge Isotope Laboratories (Andover, Mass.) and CDN Isotopes Inc. (Quebec, Canada).

Concentrated standard solutions of individual analytes and internal standards were prepared at concentrations of about 100 micrograms per milliliter $(\mu \mathrm{g} / \mathrm{mL})$ by accurately weighing about 1 milligram $(\mathrm{mg})$ of each analyte to the nearest $0.1 \mathrm{mg}$ into a $10-\mathrm{mL}$ glass volumetric flask and dissolving in methanol to volume. Some analytes were unstable in methanol so were prepared in acetonitrile (paraoxon-methyl, parathionmethyl, diazinon oxon, and all sulfonylureas) or acetone (desulfinylfipronil amide, fipronil sulfide). For MS/MS MRM parameter optimization, standards were prepared at a concentration of $1 \mu \mathrm{g} / \mathrm{mL}$ by dilution of the concentrated standards in methanol in an analytical vial. The concentrated standard solutions were mixed using a vortex mixer (for 15-30 seconds [s]) and transferred to $20-\mathrm{mL}$ amber glass storage containers. The concentrated standard solutions are stored in a refrigerator at $4{ }^{\circ} \mathrm{C} \pm 2{ }^{\circ} \mathrm{C}$ and are stable for a least 1 year.

Internal standard (IS) solution.-The internal standard solution consists of isotope-labeled pesticide compounds at varying concentrations from 6 to 800 nanograms per milliliter ( $\mathrm{ng} / \mathrm{mL}$ ) (table 2). These labeled compounds are used as internal standards for quantitation of method analytes. Their recovery is calculated relative to a single internal standard (atrazine- $\mathrm{d}_{5}$ in $\mathrm{ESI}+$; ibuprofen-13C in $\mathrm{ESI}-$ ) to provide matrixrelated method-performance information, similar to a surrogate analyte. The concentration of each labeled analyte in the solution was selected at about the mid-point of the calibration range to provide good analytical signal. We found that trace amounts of pyrimidinol, a degradate of diazinon-d10, were present in the neat labeled standard of diazinon-d10 as an impurity and caused measurable concentrations of pyrimidinol in samples, so the concentration of this IS was lowered to try to minimize this contamination.

The IS solution is prepared by transferring appropriate volumes of the $100-\mu \mathrm{g} / \mathrm{mL}$ standard solutions to a $50-\mathrm{mL}$ volumetric flask and diluting to volume with methanolacetonitrile (80:20). The IS solution is mixed using a vortex mixer (15-30 s) and transferred to $12-\mathrm{mL}$ amber-glass storage containers. The solutions are stored in a freezer at $-10{ }^{\circ} \mathrm{C} \pm 2{ }^{\circ} \mathrm{C}$ and are stable for a least 1 year. Samples are prepared for analysis by adding $10 \mu \mathrm{L}$ of this solution to $900 \mu \mathrm{L}$ of sample in an analytical vial, resulting in expected nominal concentrations of the IS compounds from 60 to $10,000 \mathrm{ng} / \mathrm{L}$ (table 2).

Mixed intermediate standard solutions.-Pesticide compounds were divided into 24 main analytical groups consisting of 1-31 pesticide compounds per group. Analytical groups were based on pesticide use (herbicide, insecticide, fungicide), chemical class (acetanilide, triazine, acid), and solvent used. Pesticides and degradates within each group were also separated to monitor the stability of pesticides and the formation of degradates during storage. Analytical groups for the mixed intermediate solutions are shown in supporting table S1. Mixed intermediate solutions of these groups were prepared by transferring appropriate volumes of the $100-\mu \mathrm{g} / \mathrm{mL}$ concentrated standard solutions to a $5-\mathrm{mL}$ or $10-\mathrm{mL}$ volumetric flask and diluting to volume with methanol-acetonitrile (50:50) to make a final concentration of 5 to $12.5 \mu \mathrm{g} / \mathrm{mL}$. Stability of the mixed intermediate standard solutions for some groups was found to be better in acetonitrile-methanol (75:25) and for diflufenzopyr in acetone/DMSO (80:20). Each mixed intermediate solution is agitated using a vortex mixer (15-30 s) and transferred to $20-\mathrm{mL}$ amber glass storage containers. The mixed intermediate solutions are stored in a freezer at $-10{ }^{\circ} \mathrm{C}$ $\pm 2{ }^{\circ} \mathrm{C}$, and are stable for a least 1 year (based on initial verification during implementation of the method).

Primary dilution standard solutions.-A solution containing all pesticide compounds was prepared by combining appropriate volumes $(140-350 \mu \mathrm{L})$ of the $5-12.5-\mu \mathrm{g} / \mathrm{mL}$ mixed intermediate solutions in a $25-\mathrm{mL}$ volumetric flask and diluting to volume 
Determination of Pesticides and Pesticide Degradates in Filtered Water

with methanol-acetonitrile $(80: 20)$ to make a final concentration of $175 \mathrm{ng} / \mathrm{mL}$. This primary dilution standard solution is used to prepare lower concentration standard solutions using digital pipettes and disposable glass pipettes to add appropriate amounts of the primary dilution standard and methanol to $2-\mathrm{mL}$ analytical vials (see supporting table S2). For example, appropriate volumes $(43-171 \mu \mathrm{L})$ of the $175-\mathrm{ng} / \mathrm{mL}$ primary dilution standard solution and methanol $(1,329-1,457 \mu \mathrm{L})$ are added to $2-\mathrm{mL}$ analytical vials to make standard solutions with concentrations of 20, 10, and $5 \mathrm{ng} / \mathrm{mL}$. Appropriate volumes of these standards are further diluted to prepare solutions with concentrations of $1,0.5$, 0.1 , and $0.05 \mathrm{ng} / \mathrm{mL}$ (supporting table $\mathrm{S} 2$ ). The primary dilution standard solutions are stored in a freezer at $-10{ }^{\circ} \mathrm{C} \pm 2{ }^{\circ} \mathrm{C}$ and are stable for at least 6 months.

Calibration standards. - Calibration standards are prepared in reagent water with methanol (10 percent) at 12 concentration levels $(1,2.5,5,10,25,50,100,250,500,1,000$, 5,000 , and $10,000 \mathrm{ng} / \mathrm{L}$ ) for each analytical batch. The calibration standards are prepared by adding appropriate volumes $(20-57 \mu \mathrm{L})$ of the $0.01-175-\mathrm{ng} / \mathrm{mL}$ primary dilution standard solutions to $900 \mu \mathrm{L}$ of laboratory reagent water in an analytical vial to make final concentrations from 1 to $10,000 \mathrm{ng} / \mathrm{L}$. A $10-\mu \mathrm{L}$ aliquot of the mixed IS solution is added to the calibration solutions, and sufficient methanol $(33-90 \mu \mathrm{L})$ is added to make the final volume $1,000 \mu \mathrm{L}$. The resulting calibration standards contain 10 percent methanol necessary for optimal chromatographic performance of early eluting analytes.

Spiking solution.-A spiking solution for preparing laboratory reagent spikes and field-matrix spikes was prepared by combining appropriate volumes $(200-1,250 \mu \mathrm{L})$ of the $5-12.5-\mu \mathrm{g} / \mathrm{mL}$ mixed intermediate solutions in a $100-\mathrm{mL}$ volumetric flask and diluting to volume with methanol-acetonitrile (80:20) to make a final concentration of $25 \mathrm{ng} / \mathrm{mL}$. This spiking solution is mixed using a vortex mixer (15-30 s) and transferred to $20-\mathrm{mL}$ amber glass storage containers. The spike solution is stored in a freezer at $-10{ }^{\circ} \mathrm{C} \pm 2{ }^{\circ} \mathrm{C}$ and is stable for a least 1 year. Reagent spikes and matrix-spike samples are prepared for analysis by accurately adding $100 \mu \mathrm{L}$ of this solution using a fixed-volume $100-\mu \mathrm{L}$ micropipettor to about $10 \mathrm{~mL}$ of sample in a sample container. The sample weight of field-matrix-spike samples is determined by weighing the filled vial to the nearest $0.1 \mathrm{~g}$. The resulting nominal concentrations of the analytes are $250 \mathrm{ng} / \mathrm{L}$.

Spike solutions for preparation of field-matrix spikes at the sample collection site are made using 2-mL flame-sealed glass ampoules. The spiking solution $(25 \mathrm{ng} / \mathrm{mL})$ is transferred to each glass ampoule using a $500-\mu \mathrm{L}$ glass syringe, and then the ampoules are sealed using an automatic ampule sealer. The ampoules must be stored in a refrigerator at $4{ }^{\circ} \mathrm{C} \pm 2{ }^{\circ} \mathrm{C}$ or freezer and used prior to their expiration date (about 1 year after preparation).

\section{Sample Collection, Filtration, Preservation, Shipment, and Holding Times}

Samples must be collected using techniques designed to collect a representative, unbiased sample. The National Field
Manual for the Collection of Water Quality Data describes protocols and provides guidelines for USGS personnel who collect water-quality data (U.S. Geological Survey, variously dated).

\section{Sample Filtration}

Stream and groundwater samples are filtered at the field site using small disposable 25 -mm-diameter syringe-tip filters (Wilde and others, 2014). About $10 \mathrm{~mL}$ of sample is pushed through the filter and collected in a glass $20-\mathrm{mL}$ sample container. A disposable $20-\mathrm{mL}$ polypropylene syringe is used to push the sample through the filter. The syringe and filter are prerinsed with about $15 \mathrm{~mL}$ of sample to clean. Alternatively, if using a pump for groundwater sampling, various reducing fittings made of PTFE and FEP can be used to connect the Luer inlet of the filter to the discharge of the pump. Other filtration procedures suitable for trace organic compounds (metal or FEP plate filters with 147-mm- and 47-mm-diameter GF/F grade glass fiber filters can also be used (Radtke and others, 2004, with updates through 2009)

\section{Field Quality Control Samples}

Collection and analysis of quality-control (QC) samples such as field-matrix spikes and field blanks are essential components of USGS water-quality field studies (U.S. Geological Survey, 2006). Matrix spikes prepared at the collection or shipping site are recommended for the DAI LC-MS/MS method in order to monitor degradation during shipping and storage as well as matrix effects because the samples are typically not preserved. Field-matrix spikes are prepared by using a $100-\mu \mathrm{L}$ micropipette to accurately add the spiking solution to about $10 \mathrm{~mL}$ of sample in a $20-\mathrm{mL}$ sample container (Sandstrom and Lewis, 2009). The sample weight is accurately determined at the NWQL and reported to the data user (NWIS parameter 3406) for calculation of spike recovery.

Field blanks are prepared using pesticide-grade water processed through sample collection, splitting, and filtration equipment.

\section{Sample Preservation}

Samples collected from sites potentially impacted by a water treatment plant that might have residual chlorine are dechlorinated with ascorbic acid. These samples should be collected in containers that contain ascorbic acid added to the container as a dry solid so that the final concentration is $0.10 \mathrm{~g} / \mathrm{L}$ ( $1 \mathrm{mg}$ for $10 \mathrm{~mL}$ of sample). Note that preservative studies described in this report show that ascorbic acid is not an ideal dechlorination reagent because it causes low recovery and higher variability for some of the pesticide compounds. Research studies are needed to identify an effective dechlorination reagent that is suitable for all pesticide compounds.

Samples collected from autosamplers require preservation or chilling to $4{ }^{\circ} \mathrm{C}$ to prevent microbial degradation while 
the sample is stored in the autosampler. One approach tested during development of the DAI LC-MS/MS method is the use of methanol as a microbial inhibiter. High concentrations of alcohol are considered toxic to most microorganisms (Malcolm Pirnie Inc., 1999), and the DAI LC-MS/MS analytical method conveniently uses methanol added to the samples at a concentration of 10 percent for optimal HPLC separation. For use as an antimicrobial, the sample containers are pre-filled with methanol so that, after filling with sample, the concentration of methanol is 10 percent, suitable for use as a microbial inhibitor. These samples do not have methanol added at the laboratory prior to analysis and so require different sample preparation steps (NWQL labcode 8237).

\section{Sample Shipment and Holding Time}

After samples are collected and filtered, they are put on ice in a cooler. Sample containers are shipped to the laboratory in ice-chilled $\left(4-6^{\circ} \mathrm{C}\right)$ coolers. Holding-time studies described in this report demonstrate that all pesticide compounds are stable for 14 days when kept chilled at $4{ }^{\circ} \mathrm{C}$. The samples should be shipped overnight to the NWQL to allow efficient management of samples to meet the 14-day holding time.

\section{Sample Preparation}

Upon arrival at the laboratory, the samples in 20-mL glass containers are stored in a refrigerator $\left(4^{\circ} \mathrm{C}\right)$ until analysis. Holding-time studies described in this report show that samples should be analyzed within 14 days of sample collection.

A batch of samples and associated QC samples and calibration samples is prepared for each analytical run. If the samples are frozen, the sample is allowed to thaw at room temperature prior to analysis (about $4 \mathrm{~h}$ ). The sample batch is prepared by sequential addition of the internal standard, methanol, and sample to the 2-mL amber glass analytical vials in parallel - each additional step is performed on all samples before moving to the next step. To prepare the samples, a digital micropipette equipped with glass bore is used to transfer $10 \mu \mathrm{L}$ of the internal standard mixture to all analytical vials, followed by $90 \mu \mathrm{L}$ of methanol to all vials. A digital pipette fitted with disposable polypropylene tip is then used to remove a $900-\mu \mathrm{L}$ aliquot of each sample from the sample container and transfer to the appropriately-labeled analytical sample vials. The methanol is used to match the organic solvent concentration (10 percent) of the mobile phase in order to optimize the peak shape of early eluting analytes in the liquid chromatographic run.

Laboratory reagent blank samples and laboratory reagent spike samples ( 1 per batch of 50 samples) are prepared in 20-mL sample containers to match the sample processing steps. These laboratory reagent blanks and reagent spikes are prepared by adding exactly $10 \mathrm{~mL}$ of the reagent water to $20-\mathrm{mL}$ sample containers. The laboratory reagent spike is prepared at an expected concentration of $250 \mathrm{ng} / \mathrm{L}$ by adding $100 \mu \mathrm{L}$ of the spiking solution to the sample in the $20-\mathrm{mL}$ sample container. Aliquots of $900 \mu \mathrm{L}$ of these samples are then transferred to the analytical vials during preparation of the sample batch. Similarly, if requested, laboratory matrix spikes can be prepared the same way as the reagent spikes by addition of the spiking solution to the environmental sample in the $20-\mathrm{mL}$ sample container. These samples, like the field-matrix spikes, have the sample weight determined by weighing the sample plus container and subtracting the container tare weight.

After data acquisition on the instrument, the original sample containers are stored in a freezer $\left(-10^{\circ} \mathrm{C}\right)$ until data analyses and reviews are completed. The glass containers will not break in a freezer as long as the sample is filled to no more than about two-thirds the capacity of the vial. In addition, when the method was developed using $40-\mathrm{mL}$ containers, placing the containers in foam sleeves prevented breakage by slowing the sample's freezing rate when transferred to the freezer, as well as when the sample was thawed.

\section{Analysis by Liquid Chromatography-Tandem Mass Spectrometry-Setup and Data Acquisition}

\section{Overview}

Samples for analysis by DAI LC-MS/MS are placed in 2-mL analytical vials with isotope-labeled internal standards and 10 percent methanol. A volume of $100 \mu \mathrm{L}$ is injected directly into the LC-MS/MS system to maximize sensitivity. The analytes are separated using an HPLC fitted with a reversed-phase column and water-methanol (ESI+) or wateracetonitrile (ESI-) gradient elution. After LC separation, the analytes are transferred in a flowing stream to the MS/MS instrument (for tandem mass spectrometric analysis) where the compounds undergo electrospray ionization in the positive or negative ion mode followed by MRM of two unique precursor to product ion transitions. Data analysis is performed using MassHunter software (Agilent), and custom reports written in Excel (Microsoft) at the NWQL are used to summarize salient analytical-run and quality-control (QC) information and prepare results for transfer to the laboratory information management system (LIMS).

\section{Separation by Liquid Chromatography}

The samples along with laboratory QC samples and calibration standards in the 2-mL analytical vials are placed in a sample tray of the autosampler, which is chilled to $4{ }^{\circ} \mathrm{C}$ by a Peltier cooling unit to minimize degradation of the analytes. A $100 \mu \mathrm{L}$ aliquot of the sample is injected into the HPLC to start chromatographic separation.

The HPLC parameters are shown in table 3. An Agilent Zorbax C18 column $(3.0 \times 100 \mathrm{~mm}, 1.8$ micrometers $)$ is used to separate analytes in both ESI+ and ESI- modes. The guard column is an Agilent 1290 Infinity in-line filter with $0.3-\mu \mathrm{m}$ 
stainless-steel frit (part no. 5067-4638). The binary mobile phase consisting of aqueous (A) formic acid/ammonium formate solution and solvent (B) methanol is used for the ESI+ mode separation. The flow rate is $0.6 \mathrm{~mL} / \mathrm{min}$ except for the initial $4 \mathrm{~min}$ where the flow rate is $0.4 \mathrm{~min}$. During method development, we determined that a slower initial flow rate gave narrower peak widths for early eluting peaks. The column temperature was set to $50^{\circ} \mathrm{C}$. The gradient for the mobile phase composition for ESI+ is shown in table 4. The total run time was $25 \mathrm{~min}$, plus $5 \mathrm{~min}$ for post-run equilibration time.

The binary mobile phase consisting of aqueous (A) acetic acid solution and solvent (B) acetonitrile is used for the ESImode separation. The flow rate is $0.6 \mathrm{~mL} / \mathrm{min}$, and the column temperature was set to $60^{\circ} \mathrm{C}$. The gradient for the mobile phase composition for ESI- is shown in table 5. The total run time was $17 \mathrm{~min}$, plus $3 \mathrm{~min}$ for post-run equilibration time.

\section{Tandem Mass Spectrometry (MS/MS) Analysis}

The LC-MS/MS system is operated in the dynamic MRM mode during data acquisition to achieve maximum sensitivity and selectivity of the MS/MS technology. In this mode, the ion source creates charged ions from the sample flowing from the LC column, and a precursor ion is selected by the first quadrupole of the MS. The second quadrupole serves as a collision cell focusing and transmitting the ions while introducing the collision gas (nitrogen) to fragment the precursor ion. The third quadrupole is used to select the characteristic fragment ions. For the method described here, two characteristic fragment ions are selected, one for quantitation and one for qualification. These characteristic precursor/product ion pairs are called MRM transitions.

\section{Development of the MRM Method}

The analytical method was first developed by selection and optimization of the MRM conditions for each analyte. The MS/MS was operated in both positive and negative electrospray ionization modes for different groups of analytes. Standards of $1 \mu \mathrm{g} / \mathrm{mL}$ in methanol were infused into the MS (no LC column) for selection of precursor and product ions and optimization of MS/MS parameters (fragmentor voltage and collision energy). Analytes were optimized in the positive ionization mode first. If response was low (integrated area of the precursor ion less than about 1,000 ) or previous literature indicated good performance in negative mode, then analytes were optimized in negative ionization mode. After MS/MS parameters were optimized for each analyte, individual standards of $0.1 \mu \mathrm{g} / \mathrm{mL}$ in reagent water were injected into the LC system with the analytical column connected to determine the analyte retention time and chromatographic performance on the LC column.

The optimized fragmentor voltages and other dynamic MRM parameters (collision energy and product ions) are shown in table 6. For most analytes in the ESI+ mode (171 of 185), the protonated molecular ion $[\mathrm{M}+\mathrm{H}]+$ was most abundant and was selected as precursor ion. For 10 pesticide compounds, the ammonia adducts $[\mathrm{M}+\mathrm{NH} 4]+$ were most abundant and selected (oxamyl, aldicarb, isoxaflutole, flumiclorac-pentyl, lactofen, piperonyl butoxide, propargite, cis-permethrin, trans-permethrin, bifenthrin). For some compounds the precursor ions were ion fragments of the molecular ion (for example, famoxadone, desiodoflubendiamide, fenbutatin oxide, fentin, terbacil). Generally, the two most abundant product ions were selected for quantitation and qualification MRM transitions, and their collision energies were optimized. For four analytes, there was only one major product ion, so the qualifier MRM selected was precursorto-precursor type (for example, $1 \mathrm{H}-1,2,4$-triazole, $70 \rightarrow 70$; methomyl oxime, $106 \rightarrow 106$; phorate oxon, $245 \rightarrow 245$; parathion-methyl, $264 \rightarrow 264$ ).

For most analytes in the ESI- mode (38 of 44), the deprotonated molecular ion $[\mathrm{M}-\mathrm{H}]$ - was most abundant and was selected as precursor ion (table 6). Generally, the two most abundant product ions were selected for quantitation and qualification MRM transitions, and their collision energies were optimized. For six pesticide compounds, there was only one major product ion, so the qualifier MRM selected was precursor-to-precursor type (for example, sec-acetochlor-OA $206 \rightarrow 206$; metribuzin DK, $183 \rightarrow 183$; sec-alachlor OA, 220 $\rightarrow 220$; hexazinone TP E, $240 \rightarrow 240$; hexazinone TP D, 224 $\rightarrow 224$; MCPA, $199 \rightarrow 199$ ).

The retention times (table 6) show that the LC conditions were sufficient to separate analytes that shared MRM transitions and provide two unique MRM transitions for all analytes. In the few cases where analytes co-eluted and shared the same precursor ion, there were unique product ions that allow identification and quantitation (for example, azinphos-methyl and phosmet, ESI+, retention time $=12.4 \mathrm{~min}$ ). The acetanilides acetochlor SA and alachlor SA were exceptions and co-elute in ESI- mode at 6.8 minutes and have common precursor and product ions. During development of the method, a number of modifications to LC conditions including column type, column temperature, and mobile phase were tested, yet all were unable to achieve separation. Additional low-abundance qualifier ions (acetochlor SA, $314 \rightarrow 162$; alachlor SA, $314 \rightarrow 160$, $314 \rightarrow 176$ ) were later added to the method to enable separate identification (table 6), at the cost of higher detection levels for these analytes.

\section{Dynamic MRM Conditions}

The dynamic MRM methods used to acquire analytical results contained 423 transitions for ESI+ mode and 120 transitions for ESI- mode. In the dynamic MRM acquisition method, the dwell times are determined by the MS/MS instrument software depending on the number of concurrent MRM transitions and retention time window set for each analyte. The retention time windows shown in table 6 were manually minimized to maintain dwell times longer than 10 microseconds for optimal signal-to-noise measurement of chromatographic peaks while still allowing for small shifts in retention time.

Once the dynamic MRM method was developed, other operational parameters were optimized by analysis of a 10,000$\mathrm{ng} / \mathrm{L}$ mixed calibration standard. These parameters were varied, 
and those that resulted in the highest response for the largest number of analytes were selected. The optimized MS/MS instrument acquisition parameters for electrospray ionization are shown in table 7.

\section{Mass Calibration}

The mass spectrometer is tuned and calibrated with the electrospray calibrant mix and procedures specified by the manufacturer. Routine use only requires resolution and mass axis verification for the resolution used in the method (Unit, Wide, or Widest resolution) and is done using the Checktune software of the instrument. The Autotune software is used to adjust and optimize ion transmission and update the electron multiplier voltage every 1 to 2 months, or if the Checktune fails. The ion response drifts lower over time, and the Autotune software restores the response.

\section{Sample Analysis Sequence}

Samples are analyzed in a sequence of instrument blanks, calibration standards, laboratory QC samples, and environmental samples called an analytical batch (table 8). A wash blank (WBLK) sample is used to verify that previous instrument cleaning was sufficient, followed by a continuing calibration blank (CCB) sample. Calibration (CAL) standards are analyzed at the beginning of each batch of samples to develop a calibration curve. Depending on the potential for carryover, one (ESI+ analytes) or two (ESI- analytes) CCB samples follow the highest calibration sample, followed by a series of continuing calibration verification (CCV) standards, about 15 environmental samples, and then by laboratory QC samples. The laboratory QC samples include laboratory reagent blanks and laboratory reagent spikes prepared in sample containers, similar to the environmental samples. Low concentration CAL standards are used as instrument detection level (IDL) standards to check LC-MS/MS response during and at the end of the analytical batch. A typical analytical batch consists of about 109 analytical vials with 75 environmental samples, 2 laboratory QC samples, and 32 instrument QC samples (blanks, calibration standards, CCVs).

The analytical batch is analyzed using an acquisition method set up with the MassHunter acquisition software. The list of samples and associated sample information for the analytical batch (created from the LIMS data system) is transferred to the LC-MS/MS prior to data acquisition to create a worklist file for data acquisition. The samples are assigned to sample types used by the MassHunter software for later data analysis (table 8). Sample vials are placed in the sample trays to match locations indicated on the worklist. The LC-MS/MS system is prepared to analyze samples by making sure adequate mobile phases are available, temperatures are set, column pressure indicates no leaks or blockage of the LC column, and the LC column has equilibrated with the initial mobile phase. Then the analytical sequence is started to acquire data for all samples. Data acquisition for a batch of 75 environmental samples plus associated QC samples takes about three days for the ESI+ mode (30 min per sample) and two days for the ESI- mode (20 min per sample).

Data users should use caution when retrieving laboratory QC data from the NWQL LIMS database because the type of the QC samples can be different from those used during data acquisition. In particular, the blanks used to check for carryover after high-calibration samples and at the start of the batch as well as the instrument blanks (IBLNK) are all identified as IBLNK-type samples in the LIMS database. The instrument blanks are most important for evaluating routine sample contamination, whereas the others are useful for evaluations of specific sources of contamination, but they should not all be combined for a summary of blank contamination that might be representative of the environmental samples.

\section{Use of Isotope-Labeled Internal Standards}

Isotope-labeled standards are used for quantitation of the pesticides using the internal standard technique. In this technique response of the pesticide relative to the internal standardmeasured as chromatographic peak area for the quantitation MRM - is determined over the calibration range. The internal standards improve quantitation because they compensate for matrix effects and injection variables compared to an external standard calibration. The internal standards cover a range of pesticide chemical classes and retention times and are chosen to best represent the diverse range of pesticides in the method.

Table 9 shows the internal standards used for the pesticide compounds. Internal standards selected were stable-isotopelabeled pesticides when available, with sufficient mass difference $(>3 \mathrm{~m} / \mathrm{z})$ between the pesticide and internal standard to avoid fragmentation of both to the same product ion. Internal standards from the same chemical class as the pesticide compounds were used if possible, although other considerations such as retention time were used to assign pesticide compounds to internal standards. Internal standards reduce the effect of ion suppression during analysis because any matrix components co-eluting with the analyte will be identical for the internal standard allowing the analyte-to-internal-standard response ratio to compensate for any ion suppression that may be present (Furey and others, 2013). However, because this method uses internal standards for analytes that elute at retention times different from the internal standard, there might be analyte responses that are not compensated for ion suppression if the matrix interference is related to a specific time of the chromatographic run. Matrix spikes are useful to monitor and identify this type of matrix interference.

The recovery of the internal standards is calculated and reported to provide a way to monitor ion suppression or enhancement of a group of analytes related to the internal standard, similar to a surrogate. Atrazine- $\mathrm{d}_{5}$ is used to calculate recovery of ESI+ mode internal standards as surrogates, and ibuprofen- ${ }^{13} \mathrm{C}_{3}$ is used to calculate recovery of ESI- mode internal standards as surrogates. 


\section{Quantitation and Calculation of Results}

Data from the analytical batch is analyzed using the MassHunter quantitative analysis software to prepare a quantitative method. The quantitative method includes information about samples and QC sample types (CAL, CCVs, blanks, and so forth) and concentrations, as well as analyte information such as whether it is an analyte, surrogate, or internal standard. In addition, analyte retention times, qualifier ion ratios, and QC limits are updated to assist in data analysis and review. The time required for quantitative analysis, peer review, and transfer of results to the LIMS for a typical batch of 75 environmental samples is about 14 days for the ESI+ mode and 6.5 days for the ESI- mode.

Calibration is performed using the peak areas and the internal standard technique. A series of $12 \mathrm{CAL}$ standards, ranging from 1 to $10,000 \mathrm{ng} / \mathrm{L}$ are analyzed at the start of each batch. The number of calibration standards used for fitting the calibration response varies from 5 to 12 , depending on the sensitivity of the analyte. The lowest calibrator used is less than the LRL. Calibration curves are generated using the MassHunter software using quadratic curve fit, ignore origin, and $1 / x$ weighting settings. An example of a calibration curve is shown in figure $1 A$. The $1 / \mathrm{x}$ weighting is used to provide a better fit of the curve and less bias at the lowest concentration CAL standards. There is negligible change in the bias at the highest concentration CAL standards using this weighting.

The calibration curve is evaluated by the overall linear fit of the quadratic curve (the statistical $\mathrm{r}^{2}$ value) and the bias of individual standards (measured as residuals in percent in MassHunter software). The fit of the quadratic curve must be greater than 0.990 . The bias of each CAL standard in the curve relative to the nominal concentration must be less than \pm 30 percent, except at the lowest level where bias is \pm 50 percent. In some batches, especially in the ESI- mode, a CAL standard might have larger bias, but the overall fit must still be greater than 0.990 . If the overall calibration curve criteria cannot be met, it might be difficult to achieve other data-quality requirements, such as CCV and IDL sample acceptance criteria. In that case, corrective action is taken to reanalyze the CAL standards, or perform maintenance on the LC-MS/MS instrument.

\section{Qualitative Determination}

Identification of pesticides in samples is done by evaluation of retention times and ion ratios of the characteristic MRM transitions. Expected values for retentions times and qualifier ion ratios were established when the method was developed and are usually updated when new calibration samples are analyzed. When a batch of samples is analyzed, the MassHunter software uses peak identification criteria based on retention time and qualifier ion ratios to identify and integrate chromatographic peaks. The analyst reviews the chromatographic peaks of both quantitative and qualifier MRM transitions and verifies the identifications. An example of a chromatogram with the
MRM quantifier and qualifier ions for a calibration standard of atrazine is shown in figure $1 C$. Manual integrations might be required for peaks with low response in noisy background, or alternatively, ignoring integrated peaks that are very small and cannot be differentiated from background noise. The signal to noise ratio, measured as peak height relative to height of background signal, needs to be above 3 for peak to be integrated and accepted by the analyst.

\section{Retention Times}

The acceptance criteria for retention times were calculated from the reproducibility of the analytes spiked at different concentrations in groundwater and stream samples during development of the method. The environmental samples typically provide more variability encountered in retention time than in reagent water. An acceptance window was set as 3 times the standard deviation of the calibration standard retention time (Lehotay and others, 2008). Typical retention times and acceptance ranges of the analytes are shown in table 10. The acceptance ranges vary with retention time, from less than 0.5 percent after $7 \mathrm{~min}$, and wider acceptance ranges from 2 to 10 percent in the first $6 \mathrm{~min}$ of the analysis. These empirical determinations of retention time of the analytes determined in matrix spikes are used as identification criteria. For example, both MRM transitions must have a retention time within the mean \pm 3 standard deviations (in percent) acceptance range to be considered for qualitative identification. These retentiontime acceptance ranges are entered into the MassHunter software and are used to display outliers in quantitative analysis and review.

\section{Qualifier Ion Ratios}

The acceptance ranges for qualifier ion ratios (QIR) vary depending on the relative response of the qualifier and quantifier MRM transitions. The relative abundance is the area of the most abundant (quantifier) ion divided by the qualifier ion expressed as a percentage; the acceptance criteria is wider for ions with small area relative to the quantifier ion. General guidelines for acceptance used by European Union for identification of veterinary drug residues in food (The Commission of the European Communities, 2002) and widely used by LC-MS/MS analysts are shown in table 11.

These guidelines are generally narrower than can be achieved in all matrices and across all concentrations for many analytes, and the criteria should not be taken as absolute limits for all applications. For this reason most laboratories require complementary interpretation by an experienced analyst to determine whether the criteria are appropriate for the sample by comparison with other QC samples (European Commission, 2012). A more practical approach is to calculate acceptance criteria for QIRs determined in calibration standards and matrix spikes to better represent the desired concentrations and matrices of the samples (Lehotay and others, 2008). This approach was taken for the DAI LC-MS/MS method, and the 
$\boldsymbol{A}$

Metolachlor - 10 Levels, 10 Levels Used, 10 Points, 10 Points Used, 0 QCs

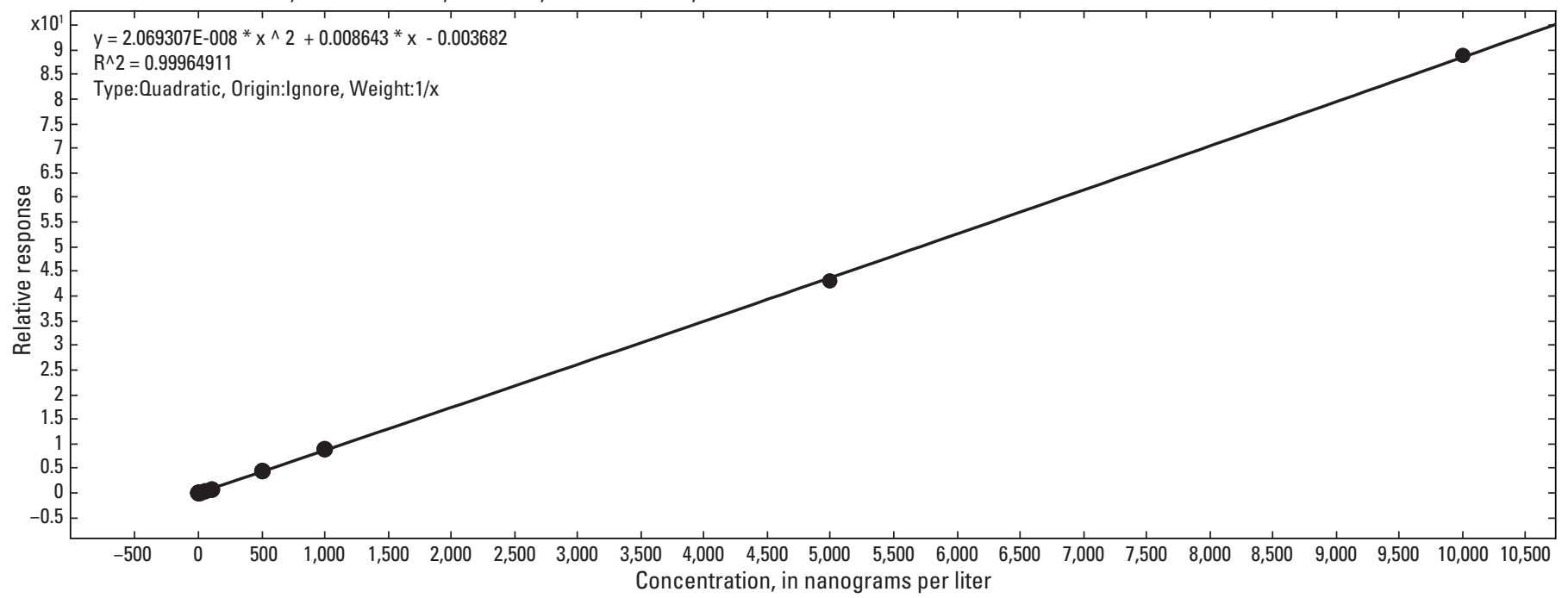

B

Metolachlor - 10 Levels, 4 Levels Used, 10 Points, 4 Points Used, 0 OCs

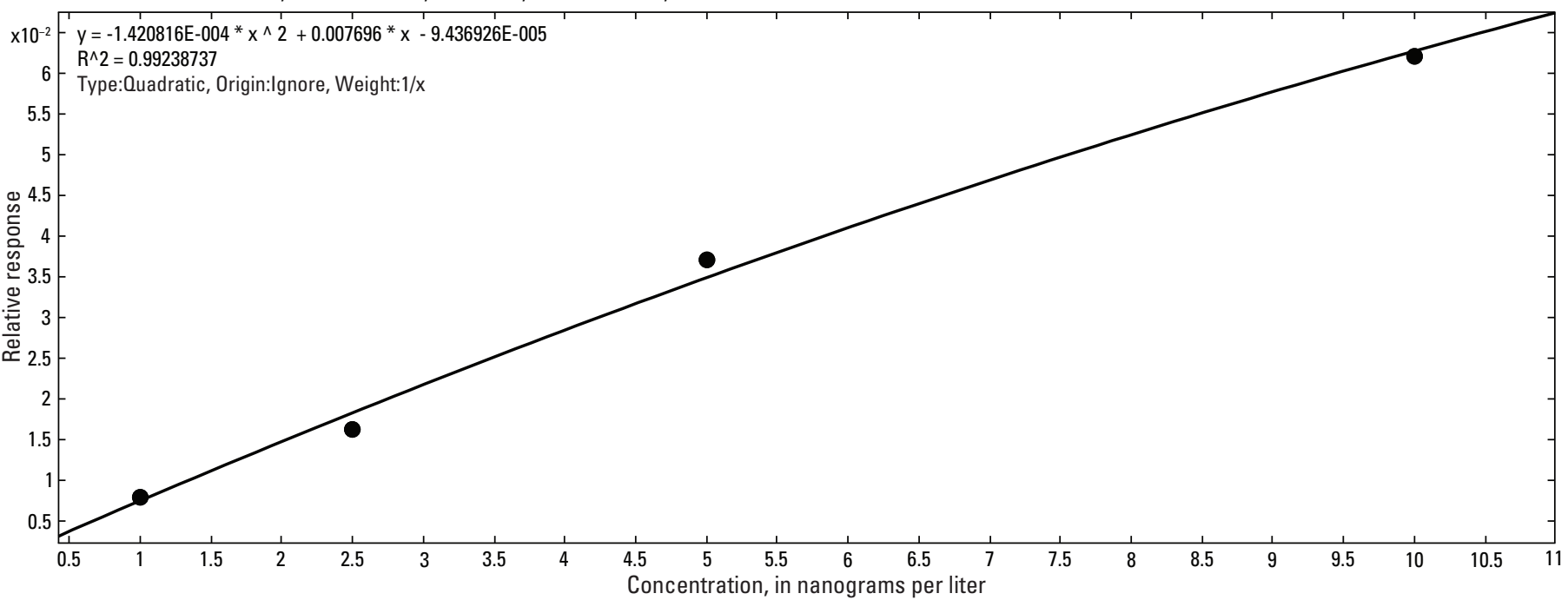

C

CAL500-1 [Metolachlor]

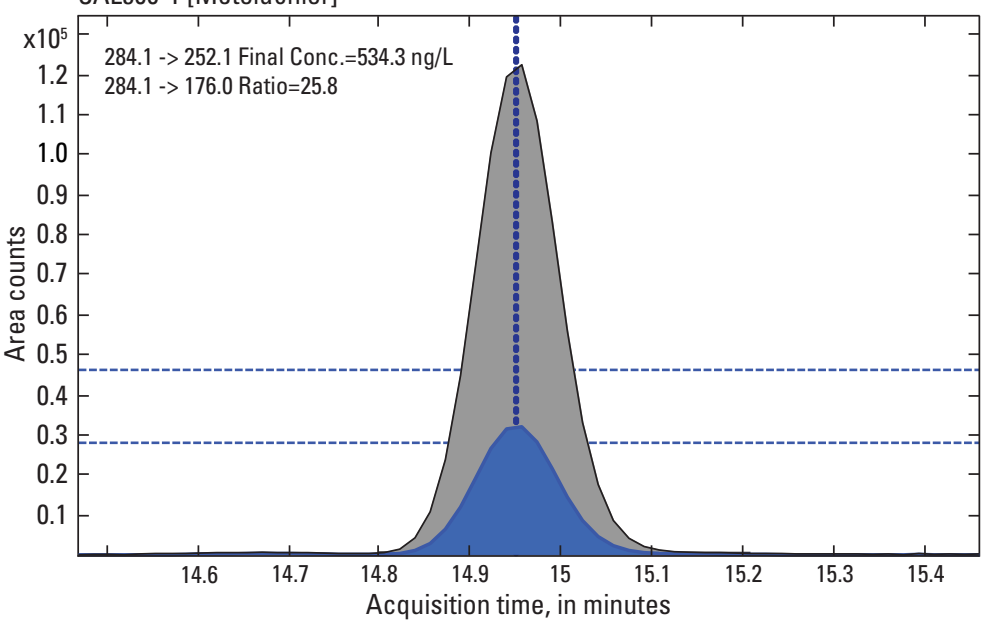

Figure 1. Example of $(A)$ a calibration curve for metolachlor determined by direct aqueous-injection liquid chromatography-tandem mass spectrometry (DAI LC-MS/MS) showing response of metolachlor relative to the internal standard metolachlor- $\mathrm{d}_{6}$ plotted as a function of concentration, $(B)$ an expanded view of the lowest section of the calibration curve, and $(C)$ low concentration standard (10 nanogram per liter [ng/L]) of metolachlor showing qualifier ( $284 \rightarrow 176$, shaded blue) and quantifier ( $284 \rightarrow 252$, shaded gray) multiple reaction monitoring (MRM) ions for metolachlor. Dotted lines show acceptance criteria for qualifier ion ratio based on \pm 15 percent. 
acceptance criteria for qualifier ion ratios determined for the pesticide compounds are shown in tables 12 and 13.

These qualifier ion ratios were determined in reagent water (laboratory reagent spike [PSPK], CCV, and CAL standards) and the three environmental matrices (two streams and a groundwater) used in the matrix validation study at four concentrations $(10,100,500$, and $5,075 \mathrm{ng} / \mathrm{L})$. The variability is larger in some cases in reagent water because this dataset included some CAL standards at lower concentrations $(<10 \mathrm{ng} / \mathrm{L})$ than the matrix samples. Statistical tolerance intervals of qualifier ion response ratios determined by DAI LC-MS/MS in both ESI+ and ESI- mode were calculated for 99 percent of the ratios (at confidence [1-alpha] of 0.95). This means that, with 95 percent confidence, the tolerance interval given by the upper and lower limits will enclose 99 percent of the QIRs. An upper limit of 75 percent and lower limit of 5 percent were used to provide useful and meaningful limits. The acceptance criteria based on the calculated tolerance intervals were used if larger than the EU limits. These empirical determinations of qualifier ion ratios are used as guides for identification criteria and are entered into the MassHunter software to help speed up initial determination of the analyte identification by displaying outliers. For example, the qualifier ion ratio must be within the acceptance criteria window to be considered for qualitative identification; otherwise, results are flagged for review by the analyst. The qualitative identification of a pesticide compound in a sample would then be based on interpretation by a skilled analyst of the comparison of ion ratios with calibration and IDL standards at a similar concentration.

Graphs of the QIRs of all pesticide compounds determined by DAI LC-MS/MS fortified at different concentrations (10, 100,500 , and $5,075 \mathrm{ng} / \mathrm{L}$ ) in the different matrices studied in the validation experiments and in $\mathrm{CAL}$ and $\mathrm{CCV}$ standards in reagent water are shown in supporting information figures $\mathrm{S} 1$ and S2. An example of a graph of the QIRs of atrazine and atrazine degradates is shown in figure 2 . These graphs show that the MRM ratios are within the EU limits at higher concentrations ( $>50 \mathrm{ng} / \mathrm{L}$ ) but exceed them at lower concentrations. The empirical-tolerance interval limits are wider and encompass most of the qualifier ion ratios at lower concentrations. Examples of the corresponding chromatograms of quantifier and qualifier multiple reaction monitoring (MRM) ions for atrazine at different concentrations (unspiked, 10, 100, 500, and 5,075 ng/L) in four different matrices are shown in figure 3 . These chromatograms show that the relative abundance of the qualifier ion is more variable in different matrices at the lowest concentration.

\section{Quality Assurance and Quality-Control Criteria}

General guidelines for acceptance criteria for QC samples, calibration, and other quality-control procedures applicable to the NWQL were used for this DAI LC-MS/MS method (Maloney, 2005). More relevant QC criteria for calibration standards, continuing calibration standards, and retention times for analyte identification were used from recent EPA methods that use LC-MS/MS for quantitative determination of organic compounds in drinking water samples (Shoemaker and Bassett, 2005; Shoemaker, 2009; Smith and others, 2011). These are summarized in table 14. If a QC sample is outside these limits, analysts might have difficulty meeting other QC requirements. Some examples of corrective actions that are taken for samples not meeting the $\mathrm{QC}$ requirements are shown in supporting table S4.

Results from this method are sometimes reported using NWIS water-quality-database remarks or value qualifier codes to qualify sample results when quality-control criteria are not met or there are other problems identified that might affect accuracy of the sample result. These codes are shown in supporting table $\mathrm{S} 3$.

\section{Routine laboratory QC Samples}

Routine laboratory QC samples include instrument QC samples (CAL standards, CCV standards, instrument blanks, third-party check standards) and batch QC samples (laboratory reagent spikes or preparation spikes [PSPK] and laboratory reagent blanks) analyzed with environmental samples. All instrument QC samples are prepared using about $1 \mathrm{~mL}$ of sample in 2-mL analytical vials. All laboratory batch QC samples are prepared using a $10-\mathrm{mL}$ sample contained in a 20-mL analytical vial.

\section{Batch OC Samples}

Laboratory reagent-water spikes and laboratory reagentwater blanks provide information about overall method performance in a control matrix and potential contamination from all processing and analysis steps in the laboratory. These QC samples are prepared with each batch of environmental samples analyzed-usually 1-2 laboratory spikes and 1 laboratory blank sample is prepared in each batch (table 8). The QC samples provide information about bias and variability due to the sample container and sample processing steps, in addition to the instrument analysis steps.

The recovery of the analytes in the laboratory reagent spikes is calculated and compared to the acceptance criteria. For the DAI LC-MS/MS method, initial acceptance criteria were set to $100 \pm 30$ percent (70 to 130 percent). Analytes with recoveries outside acceptance limits are reported to the NWIS water-quality database qualified with an "E" (estimated) remark and a value qualifier explaining that the spike failed acceptance criteria (supporting table S4) in environmental samples that have detected concentrations of the analyte.

The laboratory reagent blanks provide information about contamination during sample preparation and analysis in the laboratory. Detections of analytes in the laboratory reagent blanks are used together with instrument blanks to evaluate levels of contamination or carryover. Analytes that are detected in the blanks are reported to the NWIS water-quality database censored (reported as nondetected at the LRL) or qualified with a " $v$ " value qualifier in samples with detected concentrations, depending on the concentration in the sample relative to the concentration in the blank. 
$\boldsymbol{A}$

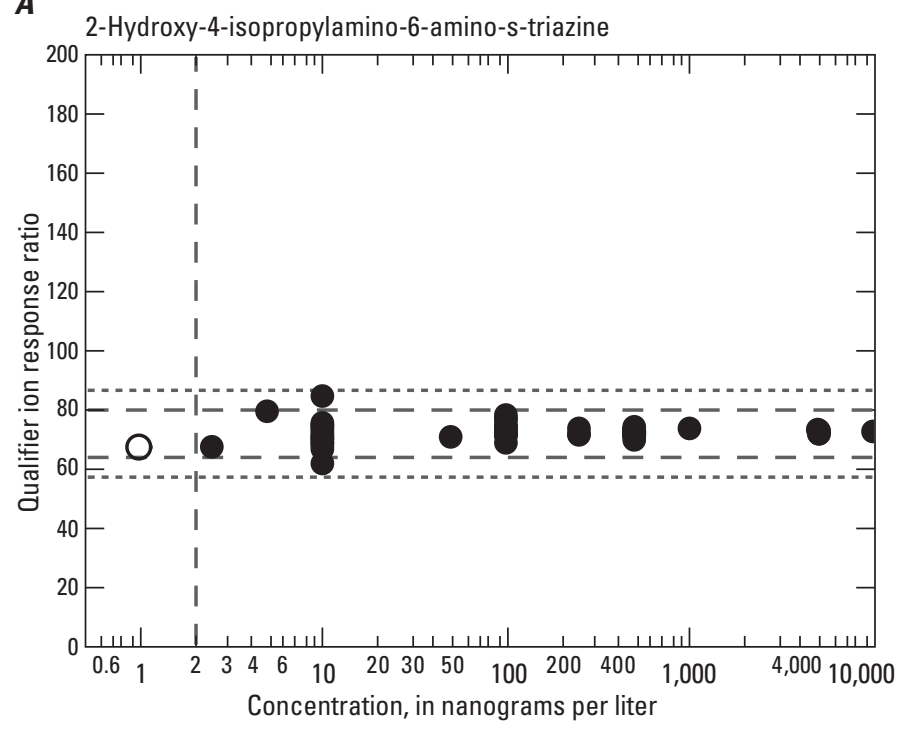

C

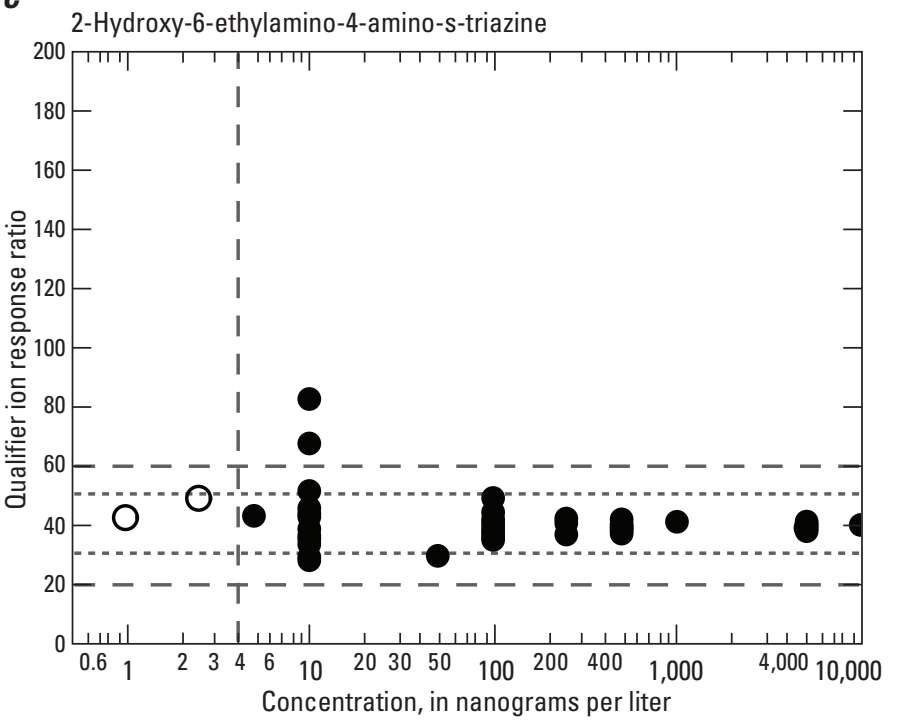

B

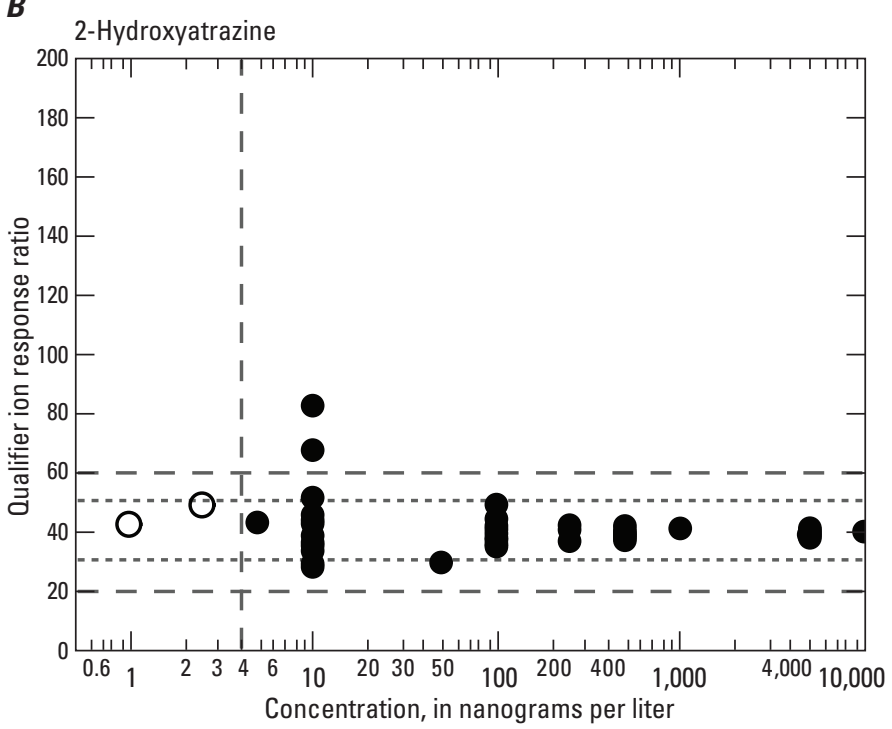

D

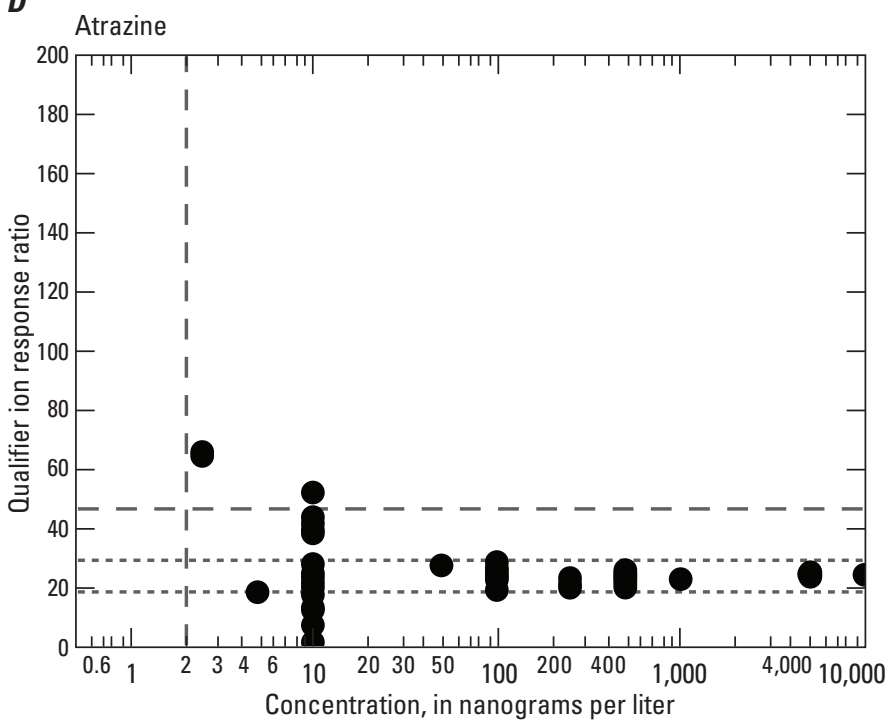

Figure 2. The qualifier ion response ratio (QIR) of atrazine and three atrazine degradates determined by direct aqueous-injection liquid chromatography-tandem mass spectrometry (DAI LC-MS/MS) in positive electrospray ionization (ESI+) mode spiked in different matrices (Santa Ana River below Prado Dam, California; Mississippi River below Grafton, Illinois; groundwater from Well 1N/14W-8K1S, Calif.) at 10, 100, 500, and 5,075 nanograms per liter ( $\mathrm{ng} / \mathrm{L}$ ) and in reagent water (calibration [CAL] and continuing calibration verification [CCV] standards at 1, 2.5, 5, 10, 25, 50, 100, 500, 5,000, and 10,000 ng/L). Samples were excluded (shown as open symbols) from evaluation if the fortification concentration was less than the method detection level (MDL). Dotted lines show European Union QIR ratio limits, and dashed lines show limits based on the statistically calculated tolerance interval. Dotted vertical line shows calculated MDL. 

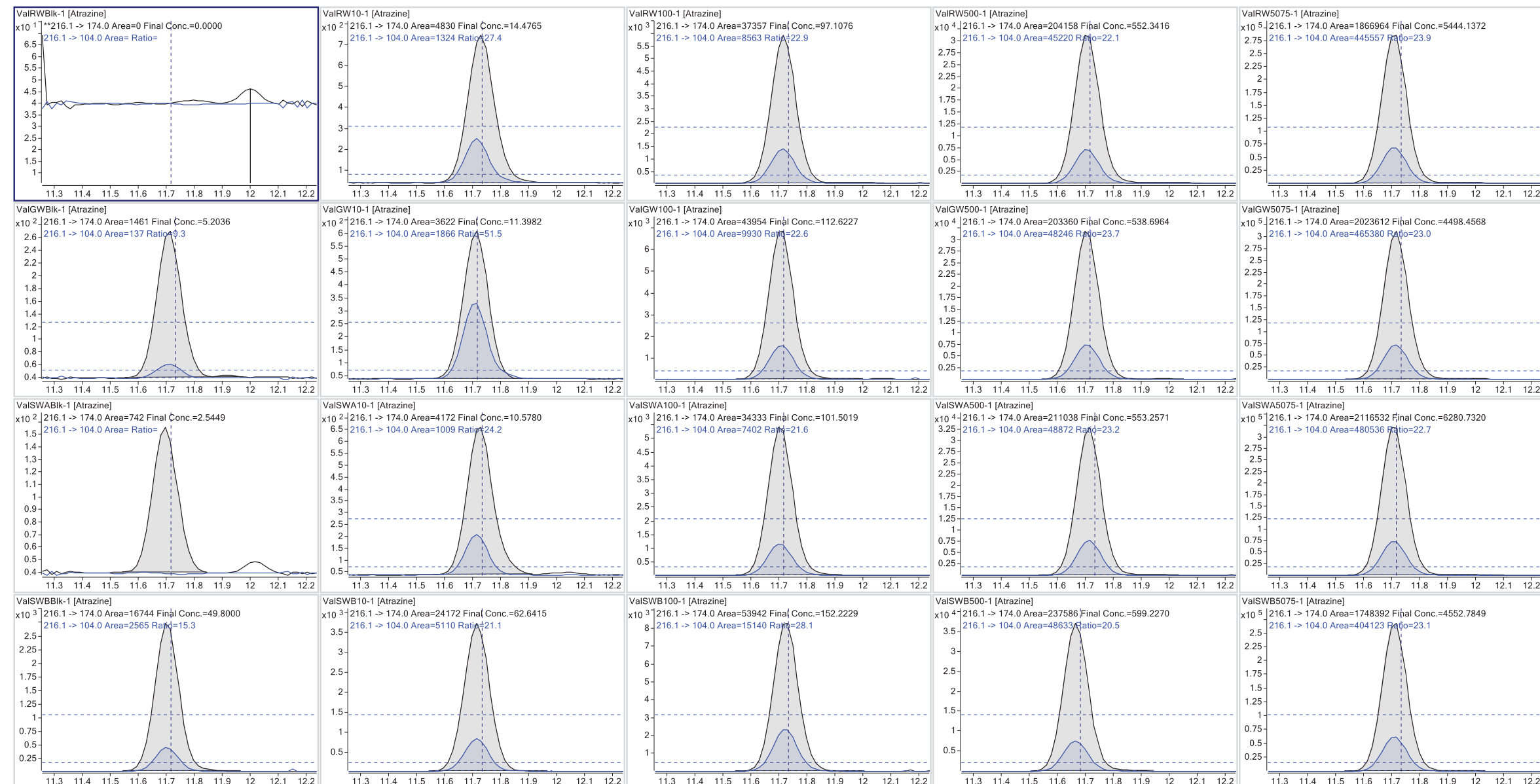

Figure 3. Chromatograms of the multiple reaction monitoring (MRM) ions for atrazine determined by direct aqueous-injection liquid chromatography-tandem mass spectrometry (DAI LC-MS/MS) at different concentrations (unspiked, 10, 100, 500, and 5,075 nanograms per liter [ng/L]) in four different matrices (reagent water, groundwater from well 1N/14W-8K1S, stream 1 [Mississippi River below Grafton, Illinois], and stream 2 [Santa Ana River below Prado Dam, California]). Quantifier ion (216 174) shown in gray and qualifier ion $(216 \rightarrow 104)$ shown in blue. Dashed lines show acceptance criteria for qualifier ion peak height relative to quantifier ion (qualifier ion ratio). Background concentrations of atrazine were 5 and $2 \mathrm{ng} / \mathrm{L}$ in groundwater well 1N/14W-8K1S and Santa Ana River below Prado Dam samples. An unidentified interference is shown in the unspiked sample from Mississippi River below Grafton, Illinois. (CNitk here to locate full-size, high-resolution image.) 


\section{Instrument Laboratory OC Samples}

Instrument QC samples include CAL standards, CCV samples, third-party check (TPC) standards, IDL standards, and instrument blanks prepared in 1-mL reagent water contained in a $2-\mathrm{mL}$ analytical vial.

\section{Standards}

Continuing calibration verification (CCV) standards are calibration standards at one concentration level analyzed throughout the analytical batch (table 8). They are used to verify continuing accuracy of the calibration curve during analysis of the entire batch of samples. Low- and mediumlevel CCVs are placed in the middle and end of the batch sequence. Acceptance criteria for recovery of all CCVs are initially \pm 30 percent and later updated based on annual summaries of CCV sample results. Analytes with recoveries outside acceptance limits are reported to the NWIS water-quality database qualified with an "E" (estimated) remark and a value qualifier explaining that the spike failed acceptance criteria (supporting table S4) for environmental samples that have detected concentrations of the analyte.

\section{Instrument Detection Level Standards}

Low-level CCVs (below the LRL) are used as IDL standards in the batch. These are analyzed at the end of the batch to verify that the detection levels are maintained during analysis of the batch of samples (table 8). The IDL is verified if all results meet qualitative identification criteria of the method that (1) both quantifier and qualifier MRM transitions are present at the correct retention time, and (2) that both MRM transitions have signal to noise ratio greater than 3 (based on visual evaluation of peak height relative to baseline). The MRM qualifier ion ratio can exceed limits that are used for qualitative identification of pesticide compounds in other non-spiked samples (for example, identification of unknowns) because the MRM ratios of the IDL are expected to be more variable at low concentrations and at the end of the analytical batch. If the IDL is not verified, there might have been a decrease in sensitivity during analysis of the batch, potentially requiring reporting all nondetections with a raised reporting level.

\section{Third-Party Check Standards}

Third-party check standards are analytical standards prepared from an independent source - different from that used to prepare the calibration and spike standards. A third-party check standard is usually analyzed with each batch of calibration standards and QC and environmental samples. During development and implementation of the DAI LC-MS/MS method, third-party check standards were not available for all analytes. However, spike solutions for NWQL schedules 2033 and 2060 contained many analytes in common and thus were suitable for use as third-party check standards. These spike standards are in ethyl acetate (2033) and methanol (2060) and can be directly added to the analytical vials. The spike mixture is diluted so that $100 \mu \mathrm{L}$ added to the $0.9-\mathrm{mL}$ reagent-water sample contained in the $2-\mathrm{mL}$ analytical vial will result in an expected concentration of $250 \mathrm{ng} / \mathrm{L}$. Acceptance criteria for recovery for all third-party check standards is \pm 30 percent. Analytes with recoveries outside acceptance limits are reported to the NWIS water-quality database qualified with an "E" (estimated) remark and a value qualifier explaining that the TPC failed acceptance criteria (supporting table S4) for environmental samples that have detected concentrations of the analyte.

\section{Reporting of Results}

Analyte concentrations in each sample are determined using the calibration curves after the MRM chromatogram has been reviewed to confirm qualitative identification of the analyte. All concentrations that meet identification criteria are reported to the NWQL LIMS, provided other quality-control criteria, primarily laboratory blank results, are met (Childress and others, 1999). Concentrations of analytes below the MDL are reported with a remark code "E" (estimated) to reflect the larger uncertainty of the concentration determined, and the appropriate NWIS value-qualifier code ("b": value was extrapolated at low end; "t": value below the MDL; supporting information tables S3 and S4). The "E" remark does not indicate that there is more uncertainty in the identification of an analyte with a concentration below the MDL because all qualitative identification criteria must be met before a concentration can be reported.

Samples with results that exceed the highest CAL standard should be either diluted or reanalyzed. In some cases where this is impractical, sample concentrations that exceed the highest CAL standard can be reported with the appropriate NWIS value-qualifier code ("a": value was extrapolated at high end; supporting information tables S3 and S4).

Final concentrations are reported in nanograms per liter rounded to the precision of the standard deviation of the replicate determinations of spike samples. For pesticide compounds with MDLs less than $10 \mathrm{ng} / \mathrm{L}$, concentrations are reported with a rounding unit of 0.1 - to 1 decimal place, but no more than 4 significant figures. For pesticide compounds with MDLs between $10 \mathrm{ng} / \mathrm{L}$ and $100 \mathrm{ng} / \mathrm{L}$, concentrations are reported with a rounding unit of $1-$ no digits to the right of the decimal place, but no more than 4 significant figures. For pesticide compounds with MDLs above $100 \mathrm{ng} / \mathrm{L}$, concentrations are reported with a rounding unit of 10 - no digits to the right of the decimal place and no significant figures between 1 and 9, but no more than 3 significant figures. Results for analytes not detected in the samples are reported as less than the laboratory reporting level $(<\mathrm{LRL})$. 


\section{Results and Discussion of Method Validation Data}

The method validation experiments consisted of studies that determined calibration linearity and response, bias and variability in different matrices at different concentrations, method detection levels, and stability of samples during storage prior to analysis and a comprehensive field study of the method performance in 47 streams, with comparison of this method to two existing NWQL pesticide analytical methods. The analytes included in the method validation were the prioritized tier 1 pesticide compounds that provided reasonable signal response under the MS/MS acquisition and chromatographic conditions used during testing in the validation studies. A number of analytes that had responses in both ESI+ and ESI- acquisition modes were tested in both modes. After initial testing, the acquisition mode providing the best response was selected. There were about 90 analytes from the initial prioritized tier 1 pesticide compounds that were not included in the method validation because they had low signal response or no retention on the reversed-phase LC system. These were mainly organochlorine, pyrethroid and dinitroaniline pesticides, as well as highly polar compounds. These analytes are shown in supporting table S5.

\section{Data-Quality Objectives}

The data-quality objectives of the validation experiments were that bias must be within 30 percent of the expected value (recovery of 100 percent \pm 30 percent) and variability less than or equal to 30-percent relative standard deviation. Analytes not meeting these objectives in more than one matrix or stability test were removed from the method. Some analytes where these objectives were not met in one matrix are reported to NWIS with an estimated remark ("E").

\section{Data Analysis}

JMP software version 9.0 (SAS Institute Inc., 2010) was used for statistical analysis. Mean, standard deviation, relative standard deviation, median, and selected quantiles were summarized for pesticide compounds in the different validation experiments. Symmetric confidence intervals for the mean were computed using the Student's t statistic. The critical values for $t$ are computed at one-half the desired level of confidence (alpha level) for a two-sided test. The width of the confidence interval is a function of these critical values, the standards deviation of the data, and the sample size (Helsel and Hirsch, 1992). Control limits for the pesticide compounds were calculated using the median and F-pseudosigma of spike recoveries. The F-pseudosigma is the interquartile range divided by 1.349 and is equal to the standard deviation of a normal distribution but is not as strongly influenced by outliers (Helsel and Hirsch, 2002).
The relative percent difference (RPD) of replicate sample results was calculated using the following equation:

$$
\frac{|e-r|}{\left(\frac{e+r}{2}\right)} \times 100
$$

where

$$
\begin{array}{ll}
e & \text { is the environmental sample result and } \\
r & \text { is the replicate sample result. }
\end{array}
$$

The RPD was not calculated if one of the sample results was missing or reported as not detected.

Specification limits for qualifier ion response ratios (QIR) were developed using tolerance intervals. A tolerance interval contains at least a specified proportion of the population; it is a confidence interval for a specified proportion of the sample population (Hahn and Meeker, 1991). Tolerance intervals of QIR were calculated for 99 percent of the ratios (at confidence [1-alpha] of 0.95). This means that, with 95 percent confidence, the tolerance interval given by the upper and lower limits will enclose 99 percent of the QIRs.

The sign test was used to compare two sets of results for paired samples in the paired-sample stream study. The sign test is a non-parametric alternative to the paired t-test for paired data, where the data are from a population that is neither normal nor even symmetrical (Miller and Miller, 1993). The sign test is used in this case to determine whether the median difference between paired observations equals 0 . The number of detections was not symmetrical in all cases, so the more powerful Wilcoxon Signed rank test could not be used (Miller and Miller, 1993), even though these test results are shown in the tables for comparison.

The $p$ value is the probability that a difference as large as or larger than that seen in the experiment would have occurred by chance alone if the treatment groups were in fact not different and depends on the experimental design, statistical test used, and variability of the data (Lovell, 2013). In this report a $p$ value less than 0.05 is used to highlight test results that might have chemical significance.

\section{Assessment of Suitability for the DAI LC-MS/MS Method}

Some analytes with very low response or limited range of calibration were identified as possible candidates for elimination from the method; five analytes (diquat; the kresoximmethyl degradates BF490-1, BF490-2, and BF490-9; and paraquat) were removed from the LC-MS/MS method prior to matrix-effects studies method for this reason. Eight other analytes (diflufenzopyr, diketonitrile isoxaflutole, dimethenamid OA, dimethenamid SA, dimethenamid SAA, fipronil, fipronil amide, and fipronil sulfide) performed better in ESI- mode and thus were removed from the ESI+ mode method (and added to the ESI- mode), whereas six analytes (chlorimuronethyl, chlorsulfuron, famoxadone, novaluron, propanil, and 
prosulfuron) performed better in ESI+ mode so were removed from the ESI- mode method (and added to the ESI+ mode). During the validation tests of bias and variability in different matrices and stability during storage, a number of analytes were found to greatly exceed quality-control requirements. These analytes were deleted from the method (shown with a "D" validation quality code in table 1), including eight analytes in the ESI+ mode (3-ketocarbofuran, 7-hydroxycarbofuran, aminopyralid, bifenazate, bifenazate diazene, flumicloracpentyl, formetanate, and phosmet oxon) and three in the ESImode (ammelide, deisopropyliprodione, and iprodione). Iprodione is unstable in methanol (Anisuzzaman and others, 2008), which is used for preparation of the calibration standards, and overall performance was highly variable even though we used iprodione- $\mathrm{d}_{5}$ as in internal standard. Other analytes had high bias and variability in one or two of the matrices tested and so were qualified with the estimated " $E$ " validation quality code (table 1). There are seventeen qualified analytes in the ESI+ mode (1H-1,2,4-triazole, 2-(1-hydroxyethyl)-6-methylaniline (HEMA), 3-hydroxycarbofuran, lactofen, asulam, bifenthrin, butralin, carbendazim, diazinon oxon, famoxadone, fenbutatin oxide, flumetsulam, naled, novaluron, oxyfluorfen, parathionmethyl, and phosmet) and five analytes in the ESI- mode (alachlor-SA, chlorosulfonamide acid, chlorthal-monomethyl, dicamba, and hexazinone TP D). Results from the validation tests for the deleted and qualified analytes are included in the following sections to demonstrate the reasons for their deletion or qualification. Deleted analytes are shown in the tables with the validation quality code " $\mathrm{D}$," whereas qualified analytes are shown in in tables with the validation quality code "E," and a different symbol in some figures.

\section{Analyte Response as a Function of Concentration}

\section{ESI+ Analytes}

The response factors (peak area divided by nominal concentration) were measured as a function of concentration to evaluate any significant change in response as a function of concentration. In addition, the response factors provide an indication of the magnitude of the signal in electrospray ionization that reflects detection levels, with higher response factors leading to lower detection levels. Response factors for the ESI+ analytes were calculated from seven replicate determinations of 10 calibration standards $(1,2.5,5,10,50$, $100,500,1,000,5,000$, and 10,000 ng/L) analyzed consecutively. An example of the response factors as a function of concentration for metolachlor and metolachlor degradates is shown in figure 4 (graphs of response factors of all ESI+ analytes are shown in supporting figure S4). A linear fit was applied to the response factors as a function of concentration (table 15; fig. 4). Response factors were excluded from the linear fit and summaries if the calibration standard was excluded from the calibration curve because qualifier ion response did not meet identification criteria (shown as open circles in the figures).
Response factors varied from 2 to 4,782, with an overall median of 255. Analytes with median response factors greater than 1,000 typically had narrow, symmetrical peaks that were well-resolved from the background and calculated MDLs (discussed in the "Method Detection Level Determination" section of this report) less than $10 \mathrm{ng} / \mathrm{L}$. Analytes with median response factors less than 10 typically were wide and difficult to identify from the background signal and had calculated MDLs greater than $10 \mathrm{ng} / \mathrm{L}$. There were 30 analytes from different pesticide groups that had median response factors greater than 1,000 (supporting table S6).

Analytes with median response factors less than 10 included some of those later deleted from the method because of poor performance (7-hydroxycarbofuran, aminopyralid), were deleted from the ESI+ mode and moved to the ESImode (fipronil, diketonitrile isoxaflutole), or qualified with an estimated remark (asulam,1H-1,2,4-triazole, oxyfluorfen, diazinon oxon, parathion methyl). Response factors for cispermethrin and trans-permethrin were low because the initial quantification MRM selected (408-355) had low response; after this experiment a different MRM (408-183) (table 6) that provided higher responses (about 500) was used for analysis.

For many of the analytes, the response factors were constant as a function of concentration over 4 orders of magnitude, with the slopes of the linear fit not significantly different than 0 (supporting table S6). In most cases, the response factors were more variable at the lowest concentrations, resulting in lower confidence that the slopes were not different than 0 (shown in bold and red highlight). For example, the response factors for metolachlor hydroxy morpholinone are about 340 at all concentration levels above $10 \mathrm{ng} / \mathrm{L}$ but varied between 20 and 600 below $10 \mathrm{ng} / \mathrm{L}$ (fig. 4), and the slope of the linear fit of response factors was significantly different from $0(p=0.0041)$. However, the overall magnitude of the change in response factor was small (slope $=0.006$ ). For most analytes, there was no decline in response factor at higher concentrations, which had been reported previously for some analytes using DAI LC-MS/MS (Greulich and Alder, 2008), perhaps due to instrumental differences in electrospray interface and (or) electronics for handling overlapping MRMs.

\section{ESI- Analytes}

The negative ion response factors (peak area divided by nominal concentration) were also measured as a function of concentration. An example of the response factors as a function of concentration for metolachlor SA and metolachlor OA determined in ESI- mode is shown in figure 4 (graphs of response factors of all ESI- analytes are shown in supporting table S7).

Median response factors varied from near 0 to about 230 , with an overall median of 9 . These are 1-2 orders of magnitude lower than the ESI+ response factors. There were six pesticide compounds with median response factors greater than 100, and these have calculated MDLs (discussed in the "Method Detection Level Determination" section of this 
$\boldsymbol{A}$

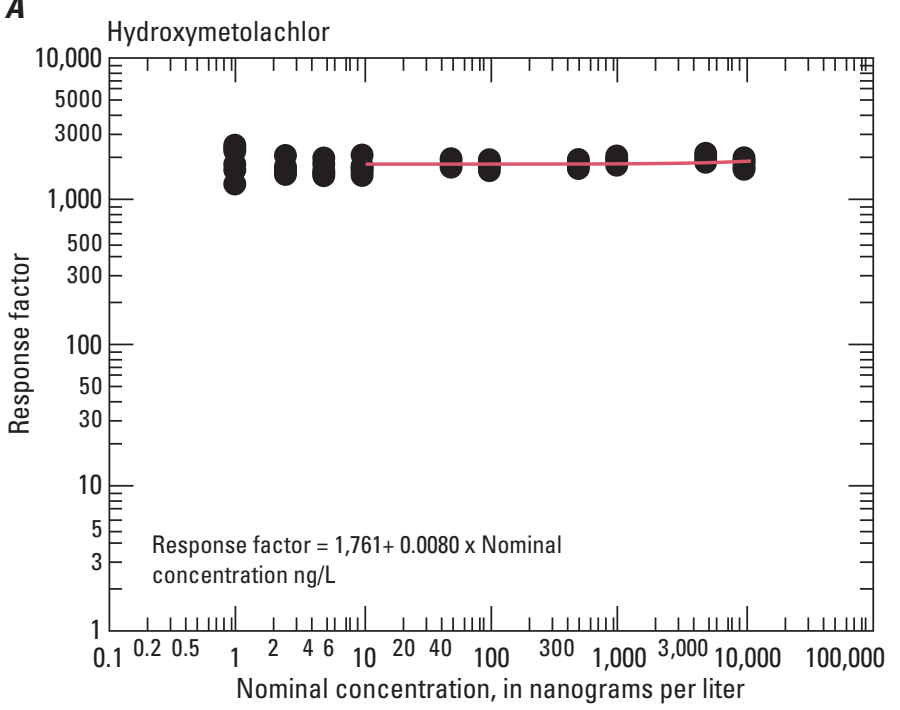

C

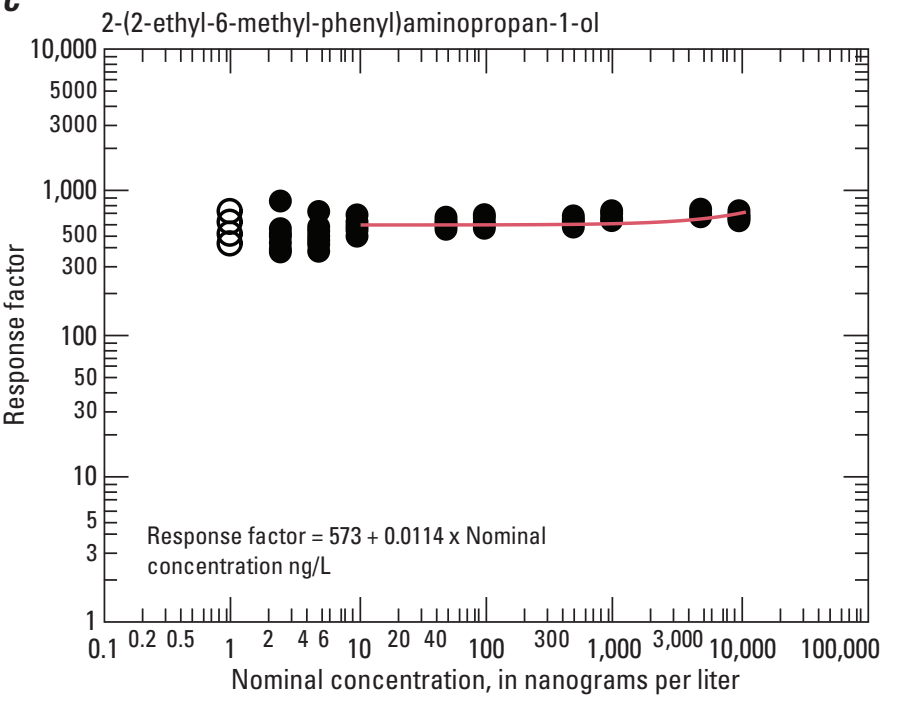

E

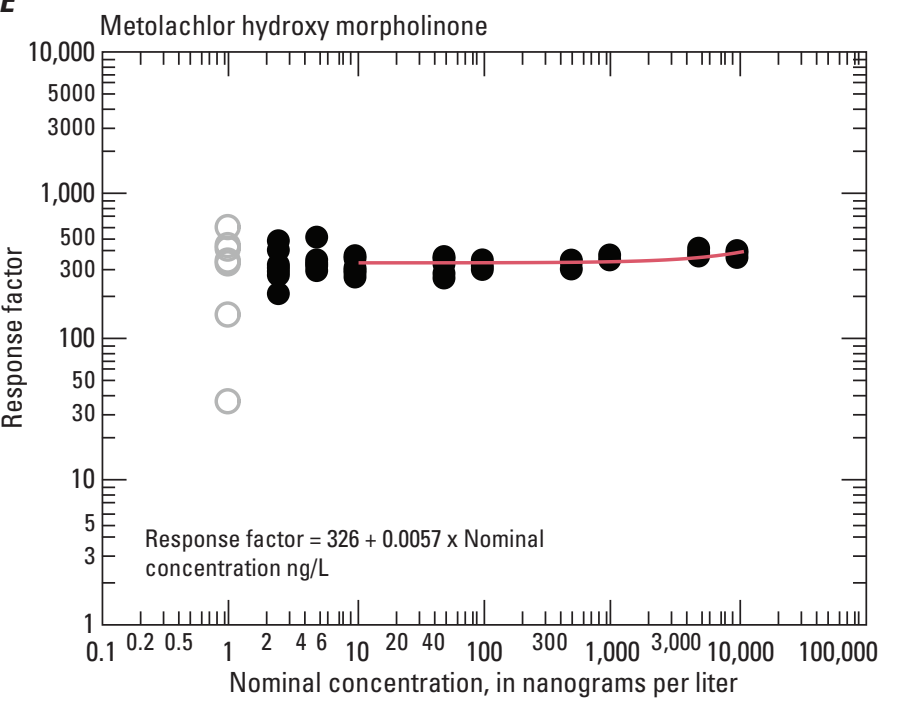

$B$

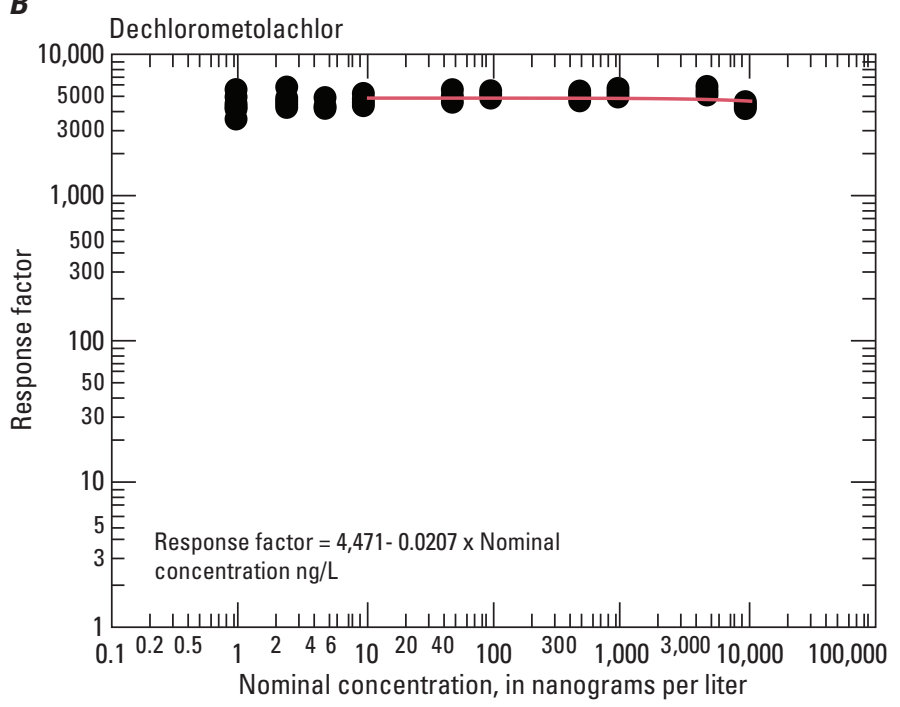

D

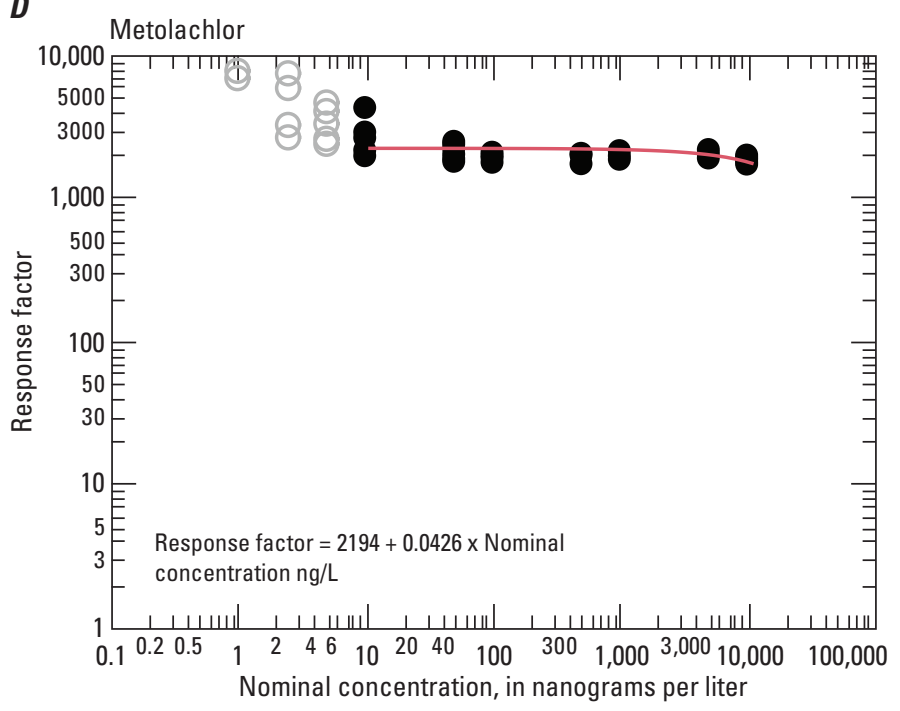

$\boldsymbol{F}$

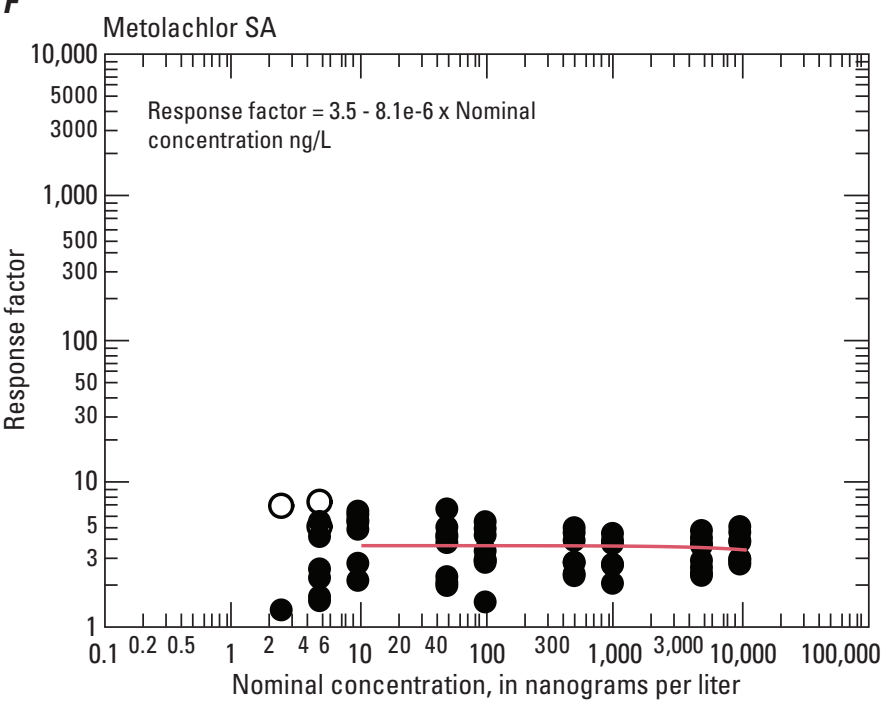




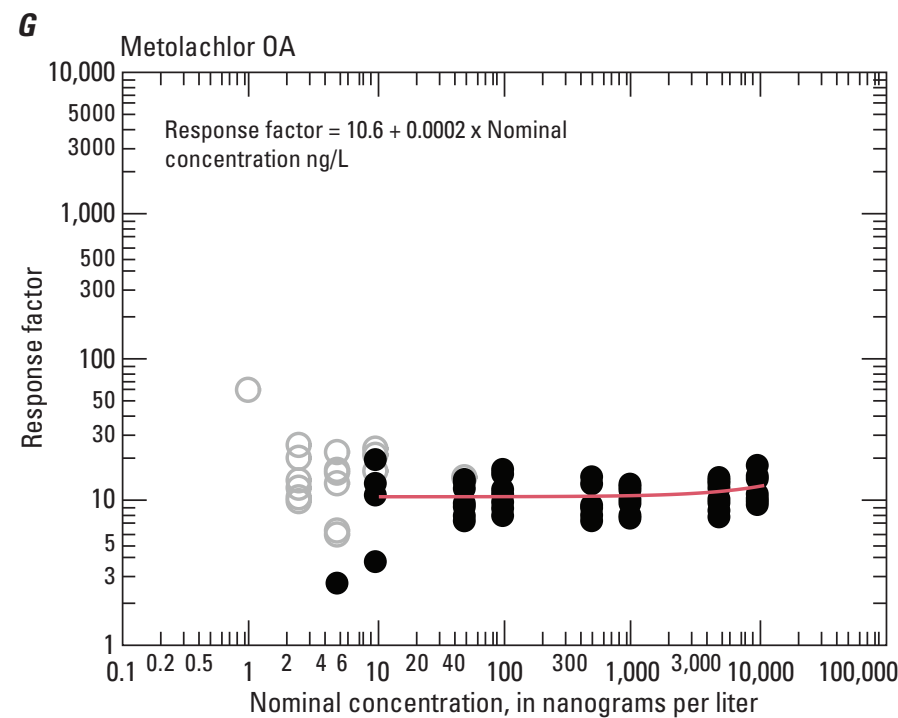

Figure 4 above and prevous page. Response factors (peak area divided by nominal concentration) as a function of concentration $(1,2.5,5,10,50,100,500,1,000,5,000$, and 10,000 nanograms per liter [ng/L], seven replicates at each concentration) for metolachlor and degradates determined by direct aqueous-injection liquid chromatography-tandem mass spectrometry (DAI LC-MS/ $\mathrm{MS}$ ) in positive electrospray ionization (ESI+) mode. Calibration standards were excluded from fit if the qualifier ion response did not meet identification criteria (shown as open circles in the figures). Metolachlor SA and metolachlor $\mathrm{OA}$ are determined in negative electrospray ionization (ESI-) mode, the other pesticide compounds in ESI+ mode.

report) less than $10 \mathrm{ng} / \mathrm{L}$. There were eight pesticide compounds with median response factors less than 1 (table 16). Pesticide compounds with very low response factors are likely to have more problems with interferences at low concentrations that prevent identification.

The response factors were constant as a function of concentration over 4 orders of magnitude, with the slopes of the linear fit not significantly different than 0 (table 13). In many cases the response factors were variable at all concentrations (for example, acetochlor SA) because of the analytical variability of the analyte.

\section{Method Detection Level Determination}

\section{Procedure}

The MDL was determined from an estimate of the standard deviation of low concentrations samples based on the EPA test procedure guidelines for analysis of pollutants (40 CFR, part 136).

The MDLs were calculated in equation 2:

$$
M D L=s \times t_{(\mathrm{n}-1,1-\alpha=0.99)}
$$

where

$n \quad$ is the number of replicate determinations at 1 to 5 times the estimated MDL,

$s \quad$ is the standard deviation of measured concentrations of $\mathrm{n}$ spike determinations, is the Student's t-value at $n-1$ degrees of freedom and $1-\alpha$ (99 percent) confidence level, and

$\alpha \quad$ is the level of significance.

When $n=49$ (seven replicates in seven batches) and $\alpha=0.01, t=2.40$, and when $n=8$ (seven replicates in one batch) and $\alpha=0.01, t=2.998$.

The LRLs are initially calculated as twice the MDL, based on Childress and others (1999). After this, the LRLs might be adjusted to higher levels if the qualitative identification of a calibration standard at LRL concentration cannot be achieved.

\section{Method Detection Level Study Design}

Replicate samples of reagent water-spiked at 5 and $10 \mathrm{ng} / \mathrm{L}$ for ESI+ analytes and at 5, 10, 50, and $100 \mathrm{ng} / \mathrm{L}$ for ESI- analytes - were analyzed to determine the MDL. Seven replicates at each concentration were analyzed in seven analytical batches over a period of 2-3 days using corresponding calibration curves for each analytical batch ( 8 analytical batches were analyzed at $10 \mathrm{ng} / \mathrm{L}$ in ESI- mode). The purpose of analyzing samples in different batches was to provide an estimate of analytical variability that incorporates more of the measurement variability that is typical for routine analyses in a production laboratory (Childress and others, 1999). For some ESI+ analytes, the $10 \mathrm{ng} / \mathrm{L}$ spike level was too low for determination of MDLs, so an additional eight replicate reagent water samples were prepared at 25,50 , and $100 \mathrm{ng} / \mathrm{L}$ and analyzed in one batch.

\section{Results of Method Detection Level Determination}

Method detection levels, LRLs, and lowest and highest calibration standards are summarized in table 17.

The calculated MDLs ranged from 1 to $103 \mathrm{ng} / \mathrm{L}$ for 183 analytes in ESI+ mode. Two analytes had MDLs of $250 \mathrm{ng} / \mathrm{L}$ (oxyfluorfen and parathion methyl). The median MDL was $3 \mathrm{ng} / \mathrm{L}$, with 75 percent of the MDLs less than $5 \mathrm{ng} / \mathrm{L}$ and 90 percent less than $11 \mathrm{ng} / \mathrm{L}$. The calculated MDLs in ESImode were about an order of magnitude higher and ranged from 2 to $106 \mathrm{ng} / \mathrm{L}$ for 42 analytes. Two analytes had MDLs of $250 \mathrm{ng} / \mathrm{L}$ (chlorthal-monomethyl and dicamba). The median MDL was $40 \mathrm{ng} / \mathrm{L}$, with 75 percent of the MDLs less than $72 \mathrm{ng} / \mathrm{L}$ and 90 percent less than $106 \mathrm{ng} / \mathrm{L}$.

These MDLs are comparable to or less than the MDLs of NWQL's existing analytical methods for pesticides. There are 70 pesticide compounds in the DAI LC-MS/MS method (schedule 2437) in common with laboratory schedule 2033 
that uses C-18 SPE and GC-MS. The median and 90th percentile MDLs for these compounds are 2.5 and $10 \mathrm{ng} / \mathrm{L}$ in schedule 2437 compared to 6.5 and $10 \mathrm{ng} / \mathrm{L}$ in the GC-MS method. There are a few exceptions; for example, EPTC, parathion-methyl, and oxyfluorfen have MDLs greater than $100 \mathrm{ng} / \mathrm{L}$ in the schedule 2437 compared to less than $10 \mathrm{ng} / \mathrm{L}$ in the GC-MS method. Phosmet, azinphos-methyl, dicrotophos, fenamiphos sulfoxide, fenamiphos sulfone, carbaryl, and carbofuran have MDLs less than $10 \mathrm{ng} / \mathrm{L}$ in schedule 2437 and between 30 and $100 \mathrm{ng} / \mathrm{L}$ in the GC-MS method. During development of schedule 2437, most of the pyrethroids tested were eliminated from further testing because of low sensitivity, except for cis-permethrin, trans-permethrin, and bifenthrin, which had a reasonable response in LC-MS/MS. Their calculated MDLs are comparable to the GC-MS method (less than $10 \mathrm{ng} / \mathrm{L}$ ). There are 37 pesticide compounds in schedule 2437 method in common with laboratory schedule 2060 that uses Carbopack SPE and LC-MS (Furlong and others, 2001). The median and 90th percentile MDLs for these compounds are 4.5 and $44 \mathrm{ng} / \mathrm{L}$ in schedule 2437 method compared to 20 and $60 \mathrm{ng} / \mathrm{L}$ in the LC-MS method (schedule 2060 MDLs for water year 2012). There are some exceptions; for example, chlorthal-monomethyl and dicamba have MDLs greater than $100 \mathrm{ng} / \mathrm{L}$ in the DAI LC-MS/MS method compared to 20 and $70 \mathrm{ng} / \mathrm{L}$ in the LC-MS method. Overall, the calculated MDLs show that schedule 2437 is suitable for determination of pesticide compounds in environmental samples.

The lowest calibration standard concentrations in table 17 are those that meet qualitative identification criteria, which vary according to ionization efficiency of each analyte and conditions of the mass spectrometer. The highest calibration standard concentration is $10,000 \mathrm{ng} / \mathrm{L}$ for most analytes, except for 1H-1,2,4-triazole, where the highest concentration calibration standard was $1,000 \mathrm{ng} / \mathrm{L}$. Usually the LRL was set at twice the MDL except for a few cases the LRL was set higher than twice the calculated MDL to make sure the lowest calibration standard was below the LRL (2-aminobenzimidazole, acephate, desisopropylaltrazine, formetanate, methidathion).

The low-concentration replicate determinations used for calculating MDLs for ESI+ mode analytes are shown in table 18. The MDLs were calculated for both the 5- and 10-ng/L concentration levels. According to the EPA procedure, MDLs are considered valid if the nominal spike concentration was 1 to 5 times the calculated MDL and if the detection frequency was greater than 50 percent. For many analytes, the calculated MDLs were similar using either the 5- or 10-ng/L concentration level because the standard deviations were similar. Constant standard deviation of the low-concentration spikes is one of the assumptions of the EPA procedure (Childress and others, 1999). The detection frequency of the low-concentration spikes is also shown in table 18. The detection frequency of low-concentration spikes (49 replicates) that have valid MDLs were from 86 to 100 percent. The MDL selected was the lowest nominal spike level that met the criteria of the EPA procedure. Other less sensitive analytes ( 28 of the 182 pesticide compounds shown in bold in table 18) required separate MDL calculation using higher concentration spikes because they had lower detection frequencies and calculated MDLs higher than the nominal spike concentration.

The higher concentration replicate determinations used for calculating MDLs for the less sensitive ESI+ mode analytes are shown in table 19. These MDLs were calculated from replicate analysis $(\mathrm{n}=8)$ of 5 to $100 \mathrm{ng} / \mathrm{L}$ standards in one analytical batch - a smaller number of replicates than the previous experiment but still valid for the EPA MDL procedure. The MDL selected was the lowest nominal spike level that met the criteria of the EPA procedure. For the analytes that have calculated MDLs less than $10 \mathrm{ng} / \mathrm{L}$, the MDLs are similar to those shown in table 18 , which shows that the variability of eight replicates determined in one batch was similar to 49 replicates determined in seven consecutive batches. In other words, the batch-to-batch analytical variability is small for the DAI LC-MS/MS method. The calculated MDLs for cis-permethrin and trans-permethrin shown in table 19 are lower than those in table 18 because a more sensitive MRM transition $(408 \rightarrow 183)$ was used for data acquisition for these analytes. The results shown in table 19 also provide summaries of bias and variability at multiple lowconcentration levels in reagent water and supplement the matrix experiment data discussed in the "Field-Matrix Spikes" section of the report.

The low concentration replicate determinations used for calculating MDLs for ESI- mode analytes are shown in table 20. The MDLs were calculated for all four concentration levels $(5,10,50$, and $100 \mathrm{ng} / \mathrm{L})$ analyzed and were considered valid if the nominal spike concentration was 1 to 5 times the calculated MDL and if the detection frequency was greater than 50 percent. For many analytes, the valid MDLs used the 50- and 100-ng/L concentration levels because the lower concentrations were not detected or had high bias and variability. The detection frequency of lowconcentration spikes (49 replicates; 57 replicates in eight analytical batches at $10 \mathrm{ng} / \mathrm{L}$ ) that have valid MDLs were from 92 to 100 percent.

\section{Bias and Variability from Matrix-Spike Recovery Tests}

The performance of the method in different matrices and over a range of concentrations was evaluated in a matrix-spike study. Recovery of all analytes spiked into four water matrices - reagent water, streams from Illinois and California, and groundwater from California - was measured at four concentration levels $(10,100,500$, and 5,075 ng/L). The study design included three replicates at the two higher levels and four replicates for the two lower levels. The purpose of separating the low and higher level concentrations in two studies was to get more information about bias a variability across a wider range of concentrations than is typically done in method development studies conducted in the USGS (U.S. Geological Survey Office of Water Quality, 2004). This design provides 14 samples in each matrix and 56 samples in all matrices. One additional set of four replicates was analyzed at $250 \mathrm{ng} / \mathrm{L}$ for $\mathrm{ESI}+$ mode 
analytes providing 60 samples in all matrices. Additional bias and variability estimates in reagent water at various concentrations are summarized in the MDL determination results (tables $18,19,20$, and 21), and at $250 \mathrm{ng} / \mathrm{L}$ in 47 different stream matrices in the "Paired-Sample Stream Study" section of the report.

The samples used for the matrix-effects study and their basic characteristics are shown in in table 21). The two stream samples from Illinois and California had similar pHs of about 8 but different specific conductivities, 537 and 1,100 microsiemens per centimeter $(\mu \mathrm{S} / \mathrm{cm})$, respectively. The groundwater sample had a $\mathrm{pH}$ of about 7.6 and a specific conductivity of $697 \mu \mathrm{S} / \mathrm{cm}$. The environmental samples have higher $\mathrm{pH}$ and specific conductivities than reagent water. The specific conductance is a measure of the ability of water to conduct an electrical current, and is a good measure of the ionic content of water which might affect the ionization efficiency of analytes in the MS electrospray interface. The $\mathrm{pH}$ of the sample - if very different than the conditions used for optimization of ionization conditions - might also affect the ionization efficiency of analytes during the elution window where the $\mathrm{pH}$ and (or) ionic strength of the mobile phase are altered. These environmental samples are typical of the water matrices intended to be used with the DAI LC-MS/ MS method. Both stream sites are part of the large river sites in the USGS National Water Quality Assessment (NAWQA) Program national fixed-site network and receive runoff from mixed sources (Rowe and others, 2013). Bias and variability of the pesticide compounds in other water matrices with very different characteristics, such as wastewater effluent, should be verified with matrix spikes.

The samples were prepared in 40-mL and 20-mL analytical vials and analyzed by LC-MS/MS in one analytical batch in each ESI mode. Concentrations of pesticide compounds determined in the unspiked environmental sample shown in tables 22 and 23 were subtracted from spike sample results to calculate recovery.

Recovery $(R)$ was calculated according to equation 3 :

$$
R=\frac{a-b}{c} \times 100
$$

where

$a \quad$ is the concentration of analyte in the spiked sample, in nanograms per liter;

$b \quad$ is the concentration of analyte in the unspiked environmental sample, in nanograms per liter; and

$c$ is the expected concentration, in nanograms per liter.

The expected concentration $(c)$ of the spike sample was calculated according to equation 4 :

$$
c=\frac{d \times e}{f}
$$

where

$d \quad$ is the concentration of analyte in spike mixture, in nanograms per milliliter; $e \quad$ is the volume of spike added, in milliliters; and

$f \quad$ is the sample volume, in liters.

\section{Qualification of Matrix-Spike Sample Results}

The large range of responses and calculated MDLs resulted in spike levels less than the MDL for some analytes in this study design with fixed concentration levels. Quantitative results for analytes at concentrations less than the MDL have larger uncertainty than at higher concentrations, so these would not be considered representative of the bias and variability of the analytes in general. Consequently, if the calculated MDL was less than the spike fortification level, the recovery results for that level were qualified by highlighting in the tables and excluded from the data summaries discussed below.

Another factor affecting the reliability of the recovery calculation was the concentration of the environmental sample relative to the fortification level. If the environmental sample concentration is greater than the spike concentration, there can be large errors in the calculated recoveries. Background environmental concentrations more than 3 times greater than the spike amount resulted in spike recoveries either much higher or lower than typical for schedule 2033 pesticides (Martin and Eberle, 2011). For the pesticide compounds in this method, graphs of recovery versus background concentration showed that recoveries were exceptionally high or low for some analytes when the environmental concentrations were equal to or greater than the spike amount. This is shown in examples of the recovery of atrazine and atrazine degradates as a function of environmental sample concentration in field-matrix spikes (fig. 5). Consequently, when the background environmental concentration was greater than the nominal spike level, the recovery results for that level were highlighted in the data tables.

\section{ESI+ Mode Analytes}

Mean recoveries of pesticide compounds in different water matrices and concentrations determined by the ESI+ mode are shown in table 22. The recoveries as a function of concentration in the different matrices for each pesticide compound are shown in supporting figure S5.

Salient features of the mean recoveries of pesticide compounds at different concentrations in different matrices are shown by the recovery of metolachlor in figure 6 . There was no major change in recovery at different concentration levels, similar to the linear trend of response factors with concentrations discussed previously. In addition, there was no obvious difference in recovery in the different matrices. The background concentration of metolachlor in the Mississippi River sample was $17 \mathrm{ng} / \mathrm{L}$, which is above the lowest spike level of $10 \mathrm{ng} / \mathrm{L}$. In this case, the calculated spike recoveries at this level were 94 percent and did not appear to be affected by the background concentration. 
$\boldsymbol{A}$

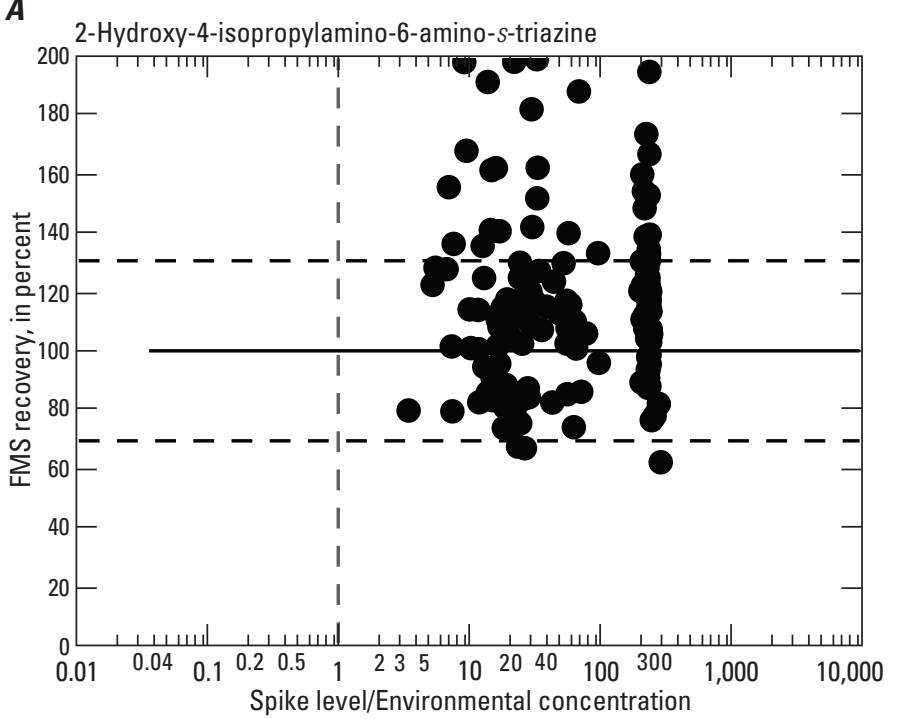

C

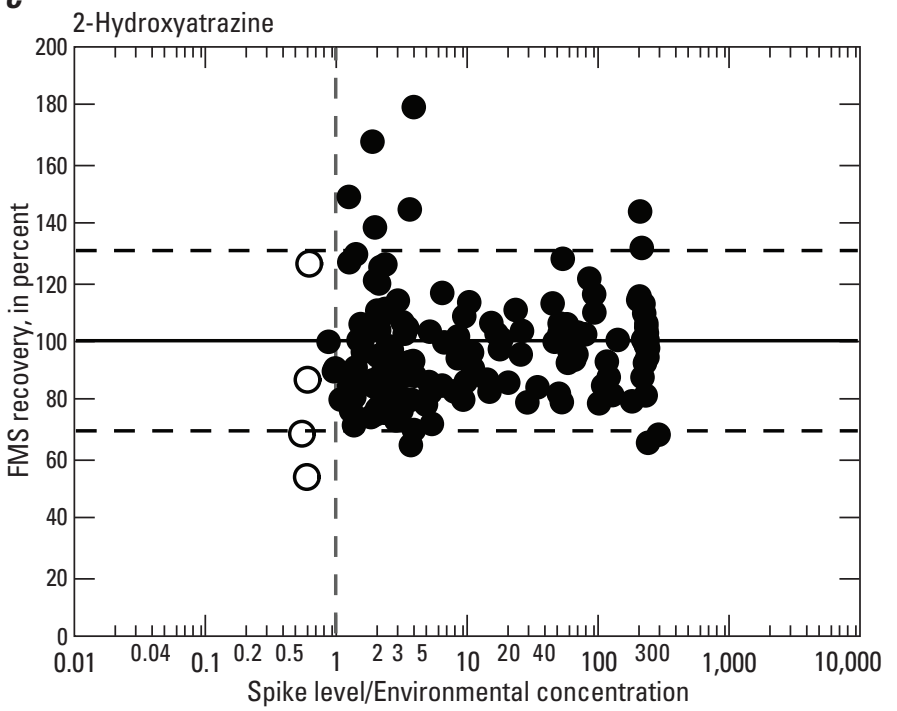

$E$

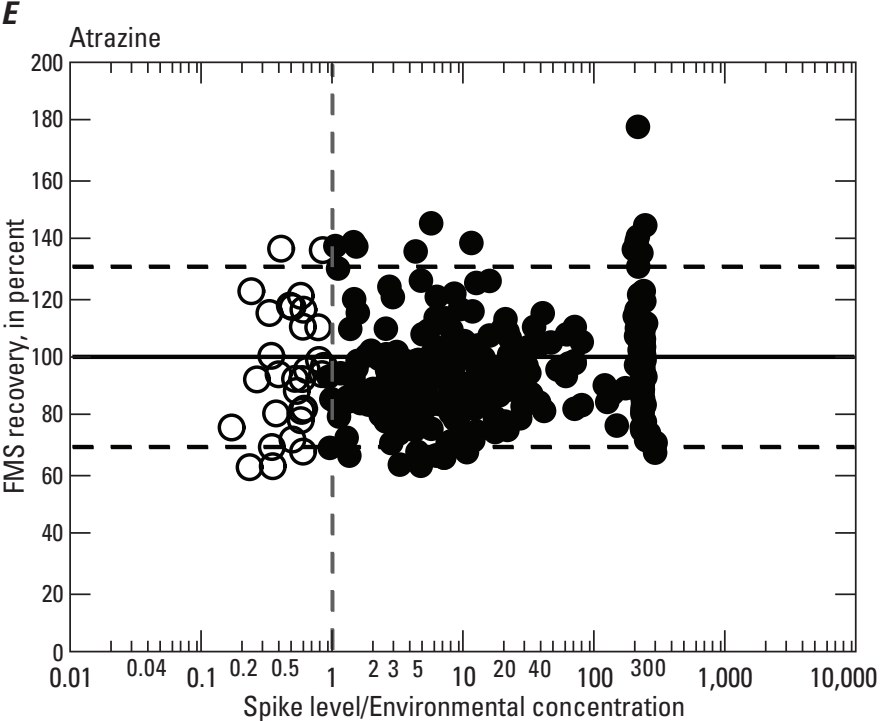

$B$

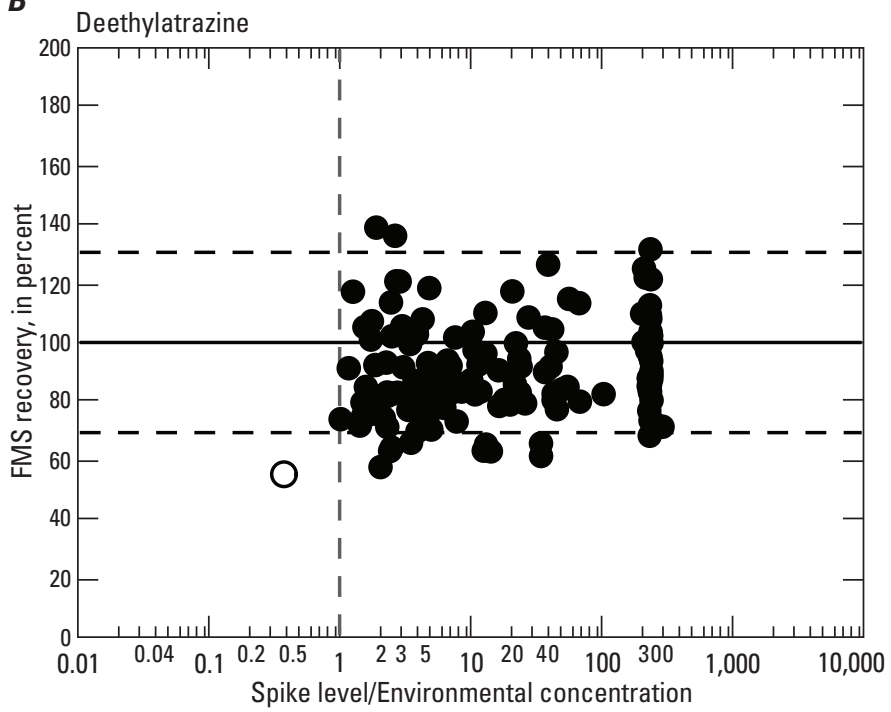

D

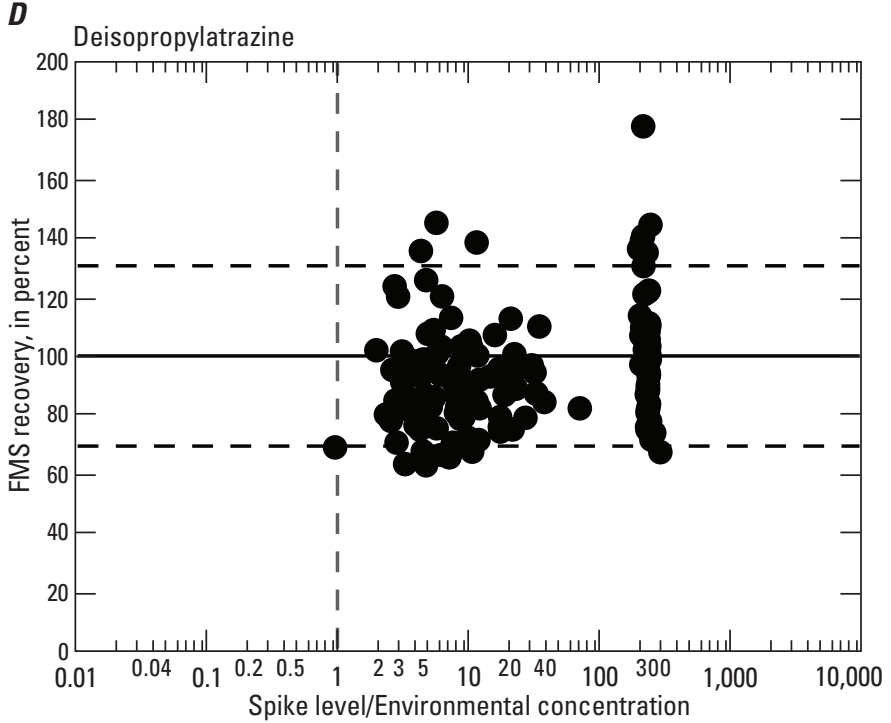

$\boldsymbol{F}$

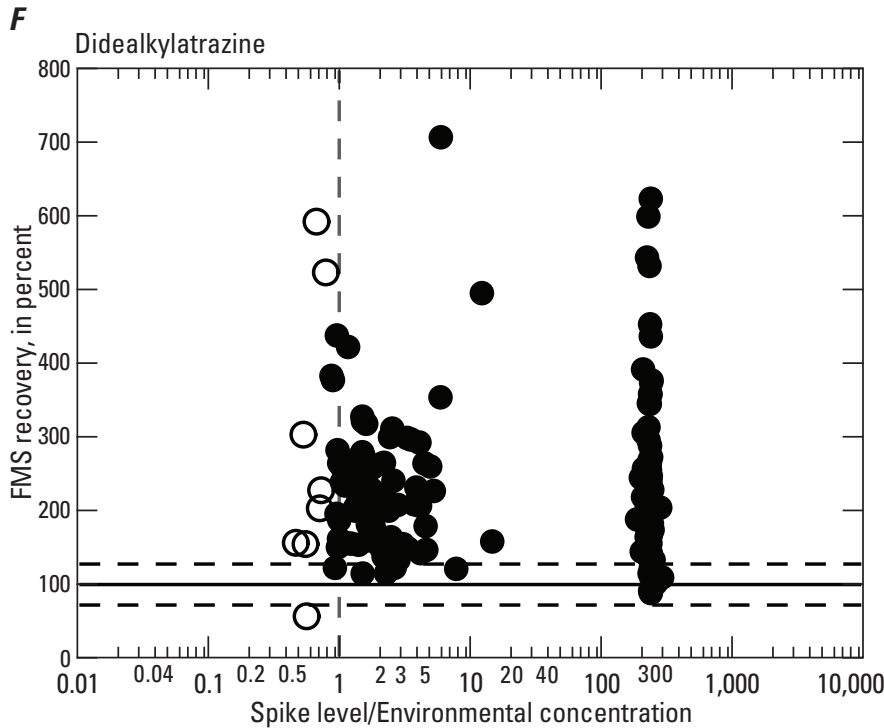


$\boldsymbol{G}$

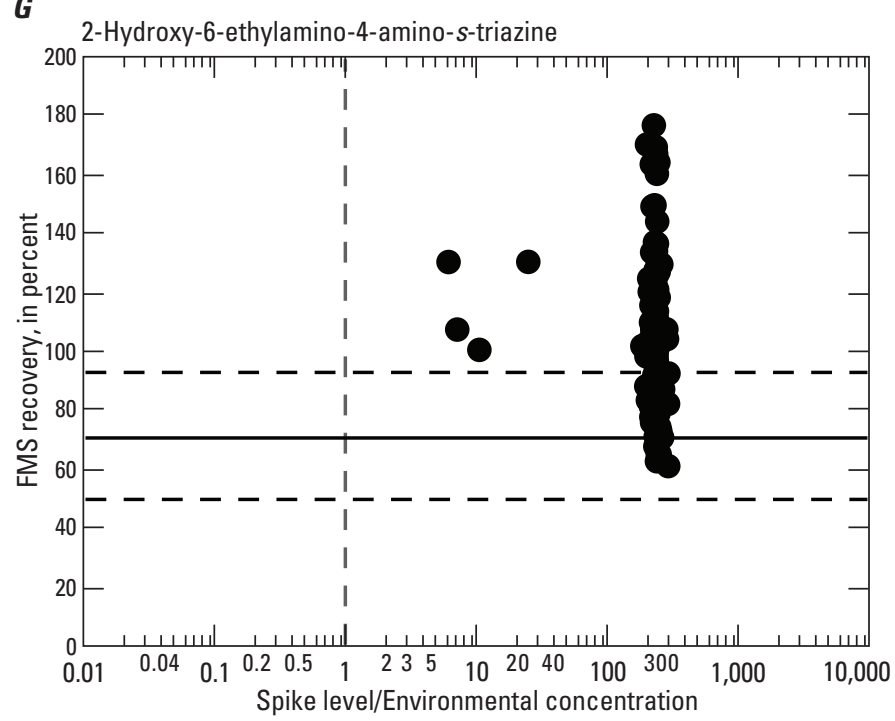

Figure 5 above and prevous page. Recovery of atrazine and degradates as a function of background environmental sample concentration determined by direct aqueous-injection liquid chromatography-tandem mass spectrometry (DAI LC-MS/MS) in 146 field-matrix-spike samples collected during the National Water Quality Assessment (NAWQA) Program field study from May to September 2012. Environmental samples, spike samples, and spike replicate samples were collected at 48 stream sites 2 to 6 times; most sites had spike samples collected 3 times and a spike replicate 1 time. Recovery of 100 percent shown as solid black line, 70 percent and 130 percent as dashed lines. Spike level/environmental sample of 1 shown as vertical dashed line. Results with nominal spike level/environmental concentration $<1$ shown as open symbols.
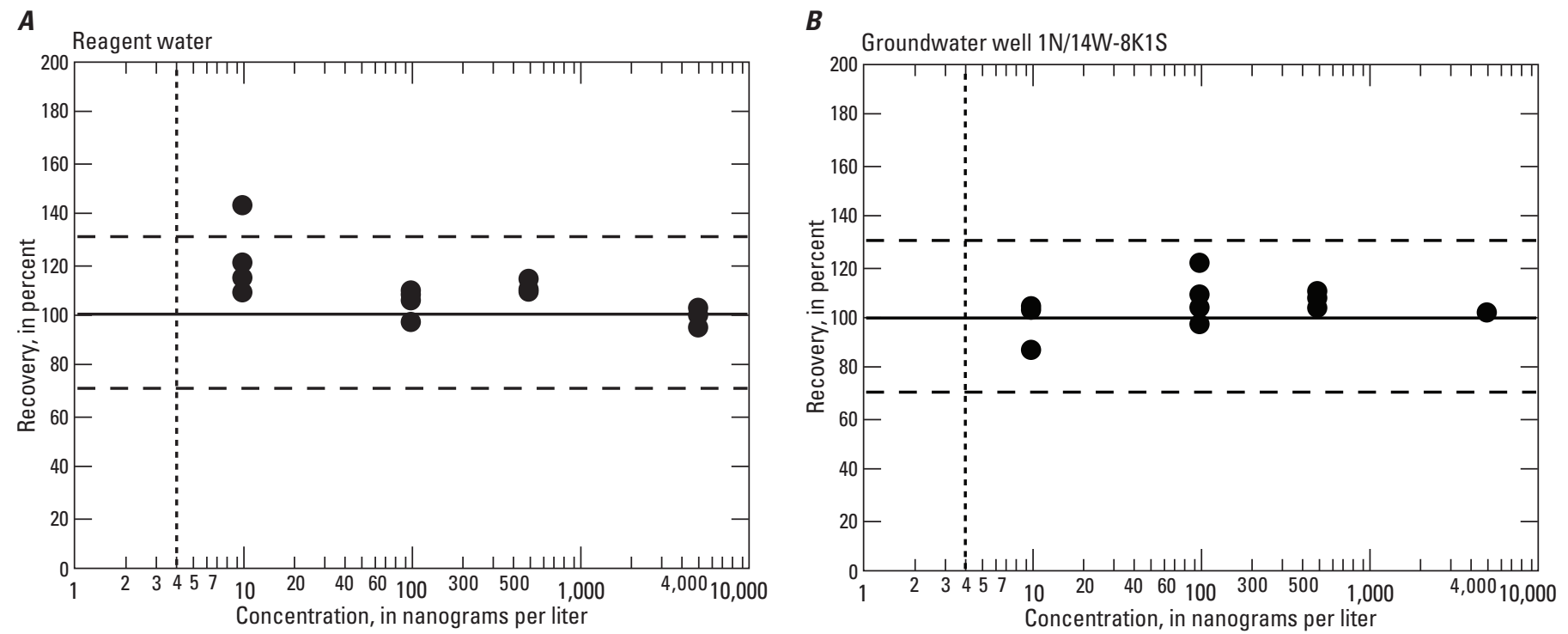
C

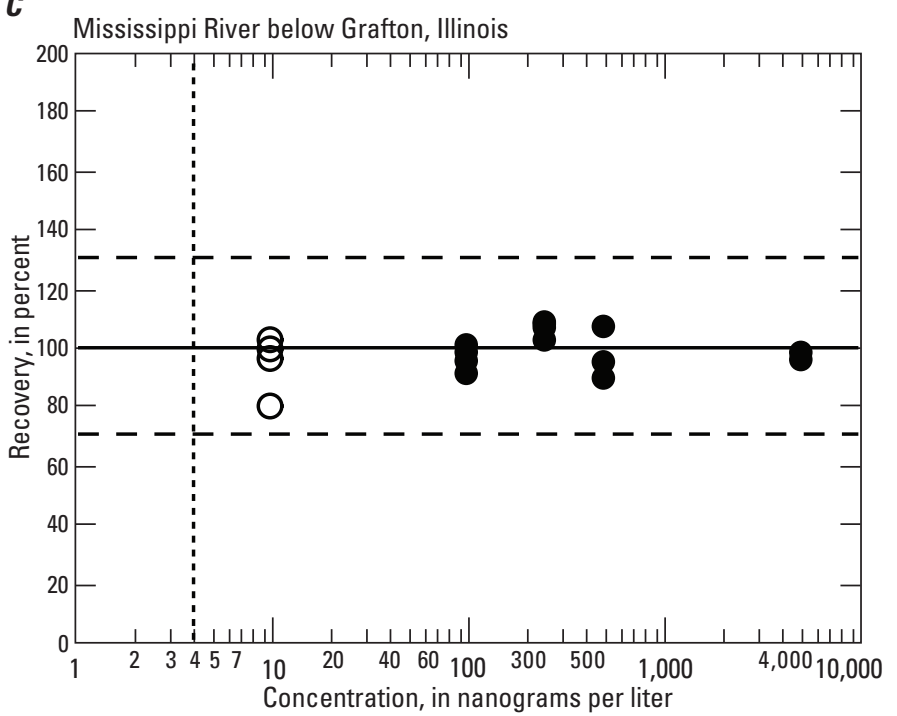

D

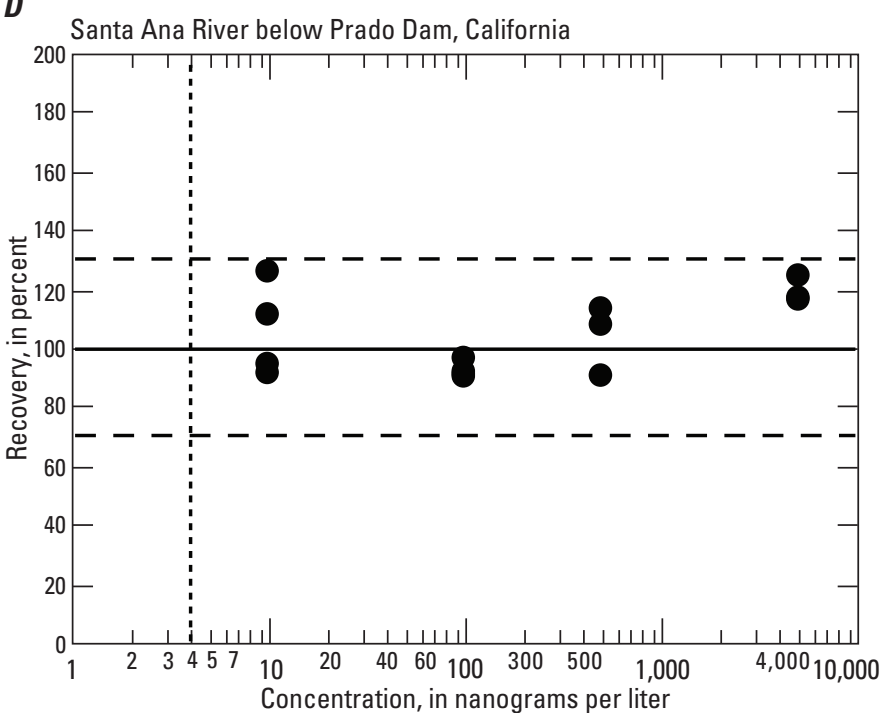

Figure 6 above and prevous page. Recovery of metolachlor determined by direct aqueous-injection liquid chromatographytandem mass spectrometry (DAI LC-MS/MS) in positive electrospray ionization (ESI+) mode in samples spiked at different concentrations $(10,100,500$, and 5,000 nanograms per liter [ng/L]) in four different matrices. Samples are shown as open symbols if the fortification concentration was less than the method detection level (MDL) or the environmental concentration. Dashed lines show study objectives of $100 \pm 30$ percent recovery. Dotted vertical line shows calculated MDL. Background concentrations of metolachlor were $17 \mathrm{ng} / \mathrm{L}$ in Mississippi River below Grafton, Illinois, samples.

Mean recoveries of most analytes in the ESI+ mode (181 of 185) were within data-quality objectives of $100 \pm 30$ percent at spike concentrations above MDLs in all four matrices (table 22). The exceptions were 1H-1,2,4-triazole, asulam, didealkylatrazine, and phosmet. Mean recoveries at medium and high concentrations $(100,500$, and 5,075 $\mathrm{ng} / \mathrm{L}$ combined; $\mathrm{n}=10)$ in reagent water were from 89 to 119 percent (median 104 percent), with relative standard deviation (RSD) less than 22 percent for 169 analytes with MDLs above the spike level. More than half (96) of these analytes had mean recoveries between 95 and 105 percent, indicating low bias for many of the analytes in the DAI LC-MS/MS method. Mean recoveries in other matrices were similar, although there were some analytes with more bias in some matrices. Analytes with slightly higher recovery in different matrices included cyanazine and imidacloprid in Mississippi River water and oxamyl oxime, imidacloprid, tebuthiuron TP 109 (OH), and 2-hydroxy-6-ethylamino-4-amino-s-triazine in Santa Ana River water. The carbamate asulam had low recoveries in all three environmental matrices but not in reagent water and thus were qualified with the permanent estimated remark. There was no obvious difference in recovery by type of pesticide (analytical method group).

Mean recoveries of the 17 surrogates in reagent water were from 90 to 109 percent, with RSD less than 20 percent. The RSDs were less than 12 percent for most of the surrogates in reagent water, except for alachlor- $\mathrm{d}_{13}$, cis-permethrin- ${ }^{13} \mathrm{C}_{6}$, and deethylatrazine- $\mathrm{d}_{6}$. Recoveries of surrogates in groundwater and Mississippi River water were similar, ranging from 95 to 100 percent and had RSDs less than 10 percent, except for cis-permethrin- ${ }^{13} \mathrm{C}_{6}$, and deetylatrazine- $\mathrm{d}_{6}$. The mean surrogate recoveries were lower and more variable in the Santa Ana River water, from 75 to 88 percent and RSD less than 35 percent, except for cis-permethrin- ${ }^{13} \mathrm{C}_{6}$ (47 percent) and deetylatrazine- $\mathrm{d}_{6}$ (66 percent). A matrix interference caused high response for the internal standard (atrazine- $d_{5}$ ) used for the surrogates that resulted in biased results for all the surrogates in these samples but did not appear to have affected any of the pesticide compounds.

Mean recoveries at the lowest spike level $(10 \mathrm{ng} / \mathrm{L})$ had more bias and variability, especially when MDLs were near the spike level. Mean recoveries at low concentrations (10 ng/L, n=4) in reagent water were from 52 (phthalazinone) to 158 percent (imazethapyr), with a median of 96 percent and RSD less than 67 percent for 155 analytes without qualification. However, most of the analytes (137) had mean recoveries within data-quality objectives of $100 \pm 30$ percent at the lowest concentration $(10 \mathrm{ng} / \mathrm{L})$. Molinate and cyanazine had mean recoveries of 0 and 2 percent, respectively, at this low level that was slightly above their calculated MDLs. Mean recoveries of disulfoton sulfone and disulfoton oxon sulfone in reagent water were high at about 220 percent, which might be related to degradation of disulfoton in the samples.

There were 18 pesticide compounds (3,4-dichlorophenylurea, carboxy molinate, chlorsulfuron, dichlorvos, didealkylatrazine, halosulfuronmethyl, hexazinone TP G, hydroxyphthalazinone, imazamox, methomyl oxime, O-ethyl-S-propyl phosphorothioate, tebuthiuron TP 106, terbacil, 1H-1,2,4-triazole, 
2-(1-hydroxyethyl)-6-methylaniline [HEMA], asulam, famoxadone, and naled) with calculated MDLs (10 to 72 $\mathrm{ng} / \mathrm{L})$ above the lowest $(10 \mathrm{ng} / \mathrm{L})$ spike-fortification level and 3 analytes (EPTC, oxyfluorfen, and parathion-methyl) with MDLs above $100 \mathrm{ng} / \mathrm{L}$ (103 to $250 \mathrm{ng} / \mathrm{L}$ ). Mean recoveries for these pesticide compounds (shown in bold highlight) were generally outside the expected data-quality objectives, with mean recoveries from 0 to 220 percent and RSD as much as 200 percent.

There were 18 pesticide compounds $(1 \mathrm{H}-1,2,4$-triazole, 2-hydroxy-6-ethylamino-4-amino-s-triazine, 2-hydroxyatrazine, atrazine, bromacil, carbendazim, carboxy molinate, deethylatrazine, deisopropylatrazine, didealkylatrazine, diuron, hexazinone, hydroxysimazine, imidacloprid, metolachlor, $\mathrm{N}$-(3,4-dichlorophenyl)-N-methylurea, simazine, and sulfometuron-methyl) that had concentrations in the environmental samples greater than the lowest spike level $(10 \mathrm{ng} / \mathrm{L})$. Recoveries for the analytes at these fortification levels were generally outside the data-quality objectives, with recoveries from -137 to 222 percent and RSD as high as 340 percent. However, when the concentration of the environmental sample was within 1 to 2 times the fortification levels, some analytes (for example, hydroxysimazine, hexazinone, 2-hydroxy6-ethylamino-4-amino-s-triazine in Santa Ana River water, metolachlor in Mississippi River water) had recoveries within the data-quality objectives.

\section{ESI- Mode Analytes}

Recovery of pesticides and degradates in different water matrices and concentrations determined by the ESImode are shown in table 23. The recoveries as a function of concentration in the different matrices for each pesticide compound are shown in supporting figure S6.

Salient features of the recovery of pesticide compounds determined in the ESI- mode by concentration and matrix is shown by the recovery of 2,4-D in figure 7 . There was no obvious difference in the recovery in the different matrices. Recoveries of analytes in the ESI- mode were more variable in general than the ESI+ mode because many of the analytes have calculated MDLs higher than the lowest spike level. The calculated MDL of 2,4-D was $31 \mathrm{ng} / \mathrm{L}$, and recoveries in reagent-water samples at the $10-\mathrm{ng} / \mathrm{L}$ spike level were from 0 to 76 percent, with a mean of 37 percent and RSD of 116 percent (recoveries were $0,0,73$, and 76 percent for each sample). Variability increased as concentration of the spike decreased. In the groundwater sample, the background concentration of 2,4-D was $4 \mathrm{ng} / \mathrm{L}$, and spike recoveries were $-44,0$, 23 , and 30 percent (mean of 2 percent, RSD of 1,509 percent). The stream samples had background concentrations of 23 and $25 \mathrm{ng} / \mathrm{L}$, and calculated recoveries at $100 \mathrm{ng} / \mathrm{L}$ (Mississippi River) and at $500 \mathrm{ng} / \mathrm{L}$ (Santa Ana River) were high (133 and 138 percent recovery, respectively).

Mean recoveries of most analytes in the ESI- mode (42 of 44) were within data-quality objectives of $100 \pm 30$ percent at spike concentrations above MDLs in all four matrices (table 23). The exceptions were fipronil sulfonate and metribuzin DK. Mean recoveries of analytes in the ESI- mode in reagent-water samples were from 93 to 136 percent (median 112 percent), with RSDs from 6 to 43 percent (39 analytes). cis-Cyhalothric acid and fipronil sulfonate had recoveries slightly higher in reagent water (134 and 136 percent), whereas dimethenamid SAA had an RSD of 37 percent in reagent-water samples. Metribuzin DK had very high recovery (353 percent) in reagent-water samples at the $100-\mathrm{ng} / \mathrm{L}$ level, the same concentration as the calculated MDL.

Mean recoveries of analytes in the ESI- mode in the other matrices were from 84 to 131 percent, with RSDs from 12 to 47 percent (39 analytes), similar to that in reagent water. Mean recoveries of most analytes $(n=38)$ were within data-quality objectives of $100 \pm 30$ percent at all spike levels, if the spike was above the calculated MDL. The mean recoveries of isoxaflutole acid RPA 203328 was more variable, with RSDs of recovery of 35 percent. Metribuzin DK had very high mean recovery (201 percent) in environmental samples at the $100 \mathrm{ng} / \mathrm{L}$ level; the same level as the calculated MDL. Mean recoveries of the fungicide famoxadone were low in both the groundwater and Mississippi River samples, so the results for this analyte are reported with an estimated validation quality code.

Mean recoveries of the seven surrogates in reagent water samples $(n=14)$ were from 99 to 109 percent, with RSD from 4 to 18 percent. The RSDs of five surrogates that have nominal spike concentrations of 1,000 to $10,0000 \mathrm{ng} / \mathrm{L}$ were less than 8 percent, whereas 2,4-D- $\mathrm{d}_{3}$ and diuron-d6, with relatively low nominal spike concentrations of $330 \mathrm{ng} / \mathrm{L}$, had higher RSD of 18 and 15 percent. Mean recoveries of each surrogate in all the environmental samples $(n=42)$ were similar to reagent water, from 100 to 111 percent, with RSDs from 4 to 19 percent. There was no meaningful difference in recovery of the surrogates in any of the environmental matrices compared to reagent water (supporting figure S2).

Most of the acetanilides had calculated MDLs above the lowest spike level. Average recoveries at medium- and high-spike concentrations were within $100 \pm 30$ percent. In the samples from Mississippi River, the background concentration of metolachlor SA was $476 \mathrm{ng} / \mathrm{L}$, yet average recoveries at 100 - and $500-\mathrm{ng} / \mathrm{L}$ spike level were within $100 \pm 30$ percent. Pesticide compounds that have calculated MDLs less than the lowest spike level were bentazon, dechlorofipronil, desulfinylfipronil, desulfinylfipronil amide, fipronil, fipronil amide, fipronil sulfide, fipronil sulfone, flubendiamide, isoxaflutole acid RPA203328, oryzalin, and sulfentrazone.

\section{Performance of Laboratory Quality Control Samples During Implementation of Method}

This method was implemented in the NWQL in June 2012 during a field comparison study of stream samples collected by the NAWQA Program. During that time, laboratory quality-control samples included third-party check standards, laboratory blank, and laboratory spike samples. 
$\boldsymbol{A}$

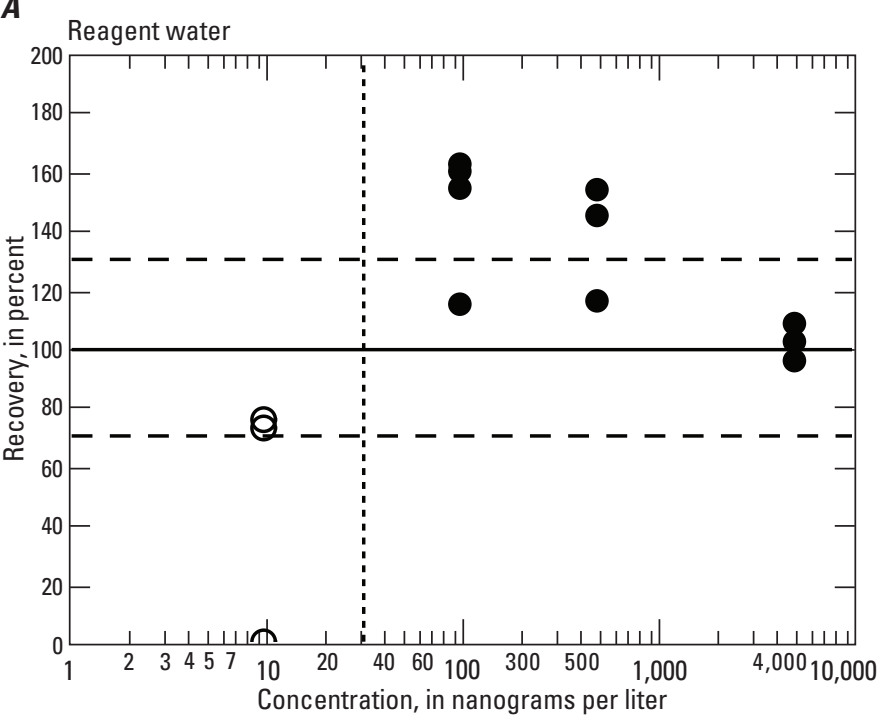

C

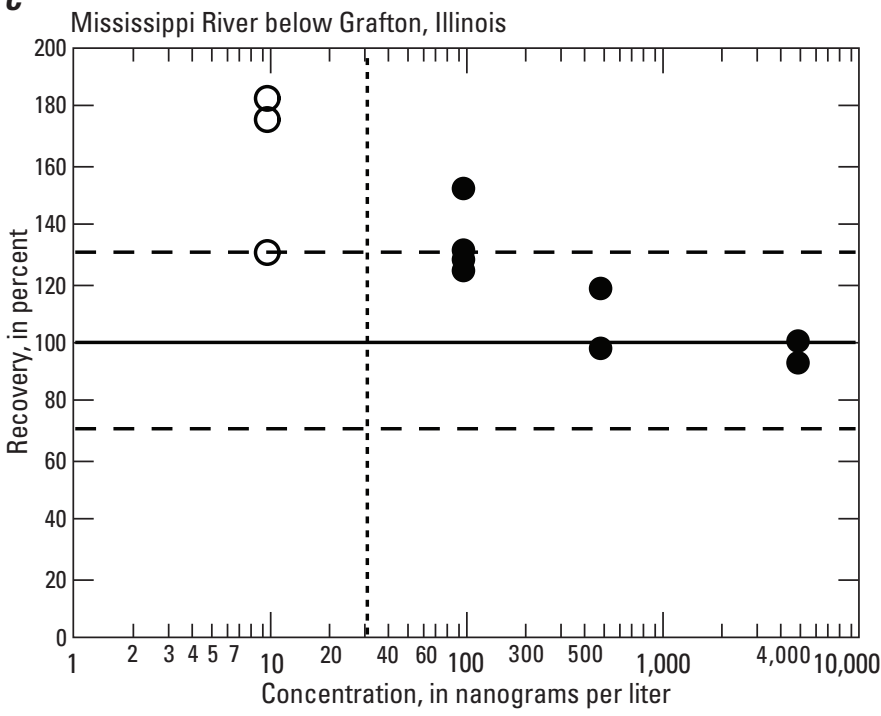

B

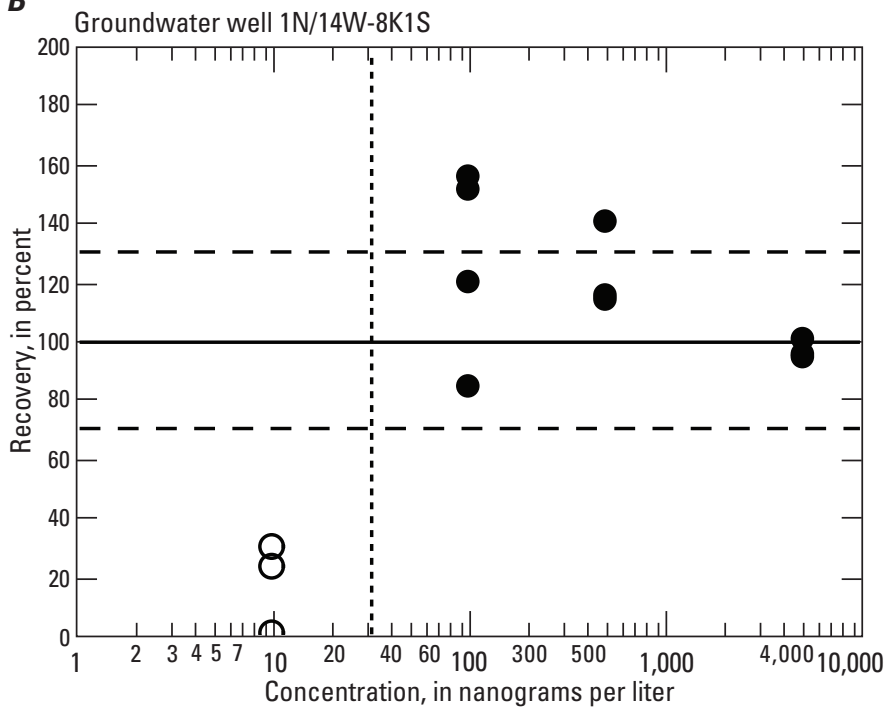

D Santa Ana River below Prado Dam, California

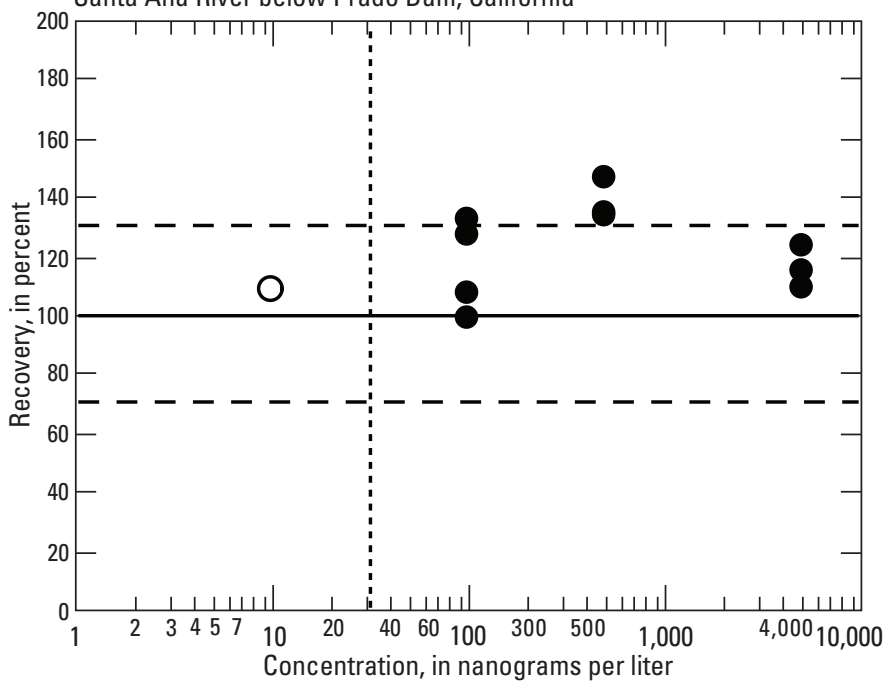

Figure 7. Recovery of 2,4-D determined by direct aqueous-injection liquid chromatography-tandem mass spectrometry (DAI LC-MS/ MS) in negative electrospray ionization (ESI-) mode in samples spiked at different concentrations $(10,100,500$, and 5,000 nanograms per liter [ng/L]) in four different sample matrices. Samples are shown as open symbols if the fortification concentration was less than the method detection level (MDL) or the environmental concentration. Dashed lines show study objectives of $100 \pm 30$ percent recovery. Dotted vertical line shows calculated MDL. Background concentrations of 2,4-D were 4, 25, and $23 \mathrm{ng} / \mathrm{L}$ in groundwater well $1 \mathrm{~N} / 14 \mathrm{~W}$ 8K1S, Mississippi River below Grafton, Illinois, and Santa Ana River below Prado Dam, California, samples. 


\section{Third-Party Check Standards}

There was no standard or group of standards commercially available that included all the analytes in the DAI LC-MS/MS method that could be used for a TPC. Spike mixtures for NWQL laboratory schedules 2033 and 2060 have 85 analytes that are common with the DAI LC-MS/MS method, including 11 analytes that are in both spike mixes. These were used as third-party check (TPC) standards during implementation of the method because they were prepared from completely independent concentrated standard mixtures. They also provide information about the differences in environmental samples and field-matrix-spike recoveries in comparison of samples analyzed by the different methods.

The TPC standard mixtures are analyzed with each batch of samples in the LC-MS/MS method. Results of TPC recovery from 11 batches of samples analyzed in the ESI+ mode between September 2012 and February 2013 are summarized in table 24. Recoveries ranged from 44 to 179 percent, with a median of 99 percent for all results. Mean recovery of each analyte in the 2033 and 2060 spike mixes was between 66 and 151 percent for all analytes without an estimated " $E$ " validation quality code, and the RSD of each analyte was from 7 to 24 percent. Most of these analytes without an estimated validation quality code ( 76 of 89 ) had mean recoveries that were between 80 and 120 percent. Ten analytes that were in both 2033 and 2060 spike mixes had comparable recoveries in schedule 2437, illustrating excellent comparability for three independent standard solutions.

A few analytes had mean recoveries outside the $100 \pm 30$-percent data-quality objectives, including organophosphate (dichlorvos, dicrotophos, fenamiphos) and carbamate (aldicarb sulfoxide) analytes. Degradation in one of the standard mixtures might be the cause of these differences because analytes in these chemical classes can be unstable during storage. Analytes reported with an estimated validation quality code had more variable recoveries (RSD from 11 to 65 percent). Benomyl, a component of the schedule 2060 spike mixture, rapidly degrades to carbendazim in water, as indicated by its quantitative recovery as carbendazim in the TPC standard analyzed by the DAI LC-MS/MS method.

Results of TPC recovery from 11 batches of samples analyzed in the ESI- mode between September 2012 and February 2013 are summarized in table 25. Recoveries ranged from 52 to 121 percent, with a mean of 97 percent and median of 98 percent for all results. Mean recovery of each of the 11 analytes (without validation quality code) in the TPC standards was between 84 and 102 percent. The RSDs of these analytes were from 6 to 22 percent. Dicamba and chlorthal-monomethyl, both qualified with " $E$ " remark in this method, had more variable recoveries, with RSD of 28 and 35 percent.

Although the TPC standards only included about one-half of the analytes in the DAI LC-MS/MS method, the analytes are from a broad range of chemical classes representative of all the analytes in the DAI LC-MS/MS method. These TPC results show that the standards used in the DAI LC-MS/MS method provide comparable results to the existing methods for those analytes for which a TPC was available. Differences in environmental samples and field-matrix-spike recovery in comparison of samples analyzed by the different methods should be less than the bias shown in tables 24 and 25 based on calibration standard differences alone. Conversely, larger differences would be due to other factors, such as matrix effects or differences in calibration, but not the analytical standards. Results for dichlorvos might be biased high in comparisons between the different methods.

\section{Laboratory Reagent Blank Samples}

Laboratory reagent blank samples are prepared with each batch of samples analyzed and are used to monitor contamination from laboratory procedures, which is most likely to occur when the sample is transferred to the analytical vial and when injected by the autosampler during data acquisition. The addition of surrogate solution can also be a source of contamination if the solution contains analytes. For this method, the standard solutions of the IS diazinon- $\mathrm{d}_{10}$ have low concentrations of the diazinon degradate pyrimidinol, so the reporting level for this analyte is generally limited by the level of the pyrimidinol from the surrogate (about $5 \mathrm{ng} / \mathrm{L}$ ). Laboratory blanks determined during implementation of the method in 2012 and 2013 are summarized in table 26. Detections of pesticide compounds in laboratory blanks were infrequent. There were 212 analytes with no detections in any lab blanks determined during the implementation studies (17 blanks for ESI+ mode, 18 blanks for ESI- mode). There were 16 analytes with detections in the lab blanks in the ESI+ mode and 2 analytes with detections in the ESI- mode. Detection frequencies were greater than 15 percent, from 18 to 35 percent (more than 3 of the 17 blanks) for fenbutatin oxide, piperonyl butoxide, and pyrimidinol in the ESI+ mode and for fipronil sulfone in the ESI- mode. The 95th percentile of concentration for most pesticide compounds in the lab blanks was less than the LRL.

These results show that contamination from laboratory procedures is unlikely for most of the pesticides in the method. The four pesticide compounds with detection frequencies above 18 percent should be carefully monitored, and if blank detections continue at a comparable frequency, they must be qualified with a " $v$ " code for detections in environmental samples.

\section{Laboratory Reagent Spike Samples}

Laboratory reagent spike samples are prepared with each batch of samples analyzed and are used to monitor performance of the analytes in a relatively matrix-free sample - in addition to the CCV samples. The laboratory reagent spikes are added to sample collection containers and are directly comparable to samples and field-matrix spikes which are collected in the same containers, unlike CCV samples that are added to 2-mL analytical vials. Laboratory spikes determined during implementation of the method from June 2012 to March 2013 are summarized in table 27. 
The mean spike recoveries in laboratory reagent spike samples for analytes in the ESI+ mode were from 66 to 114 percent, with an overall mean of 92 percent. The RSDs were from 7 to 31 percent. Mean spike recoveries in laboratory reagent spike samples for analytes in the ESI- mode were from 74 to 119 percent, with an overall mean of 93 percent. The RSDs were less than 30 percent in the ESI- mode, except for a few analytes (acetochlor SA, alachlor SA, 4-hydroxychlorothalonil, and metribuzin DADK), with RSD between 31 and 59 percent. Overall results were very good, with mean recoveries for most analytes between 80 and 120 percent and a few analytes (indoxacarb, lactofen, tribufos, cis-permethrin, trans-permethrin, chlorimuron-ethyl, orthosulfamuron, prosulfuron, and fipronil sulfone) having slightly lower mean recoveries between 66 and 80 percent. Analytes qualified with the estimated remark shown in table 27 had recoveries outside the objectives of $100 \pm 30$ percent and RSDs greater than 30 percent. Exceptions were the pesticide compounds within the objectives in the reagent-water-spike samples but with low recoveries or high bias in other matrices (asulam, 3-hydroxycarbofuran, 1H-1,2,4-triazole, butralin, flumetsulam, dicamba, and chlorosulfonamide acid).

The nonparametric statistical equivalents of the mean recovery and RSD_median and F-pseudosigma (Helsel and Hirsch, 2002) — are also shown in table 27. The mean and median recoveries are generally equivalent. The RSDs and F-pseudosigma also are similar, except in a few cases where the RSD is much larger than the F-pseudosigma. For example, hydroxytebuthiuron in the ESI+ mode had 0 percent recovery in both samples in one batch. This resulted in an RSD of 30 percent, whereas the F-pseudosigma was 14 percent because the nonparametric calculation is resistant to a few outliers compared to the parametric summary. Both statistical summaries are available from the NWQL QC sample-data application (http://nwqlqc.cr.usgs.gov/).

\section{Application to Environmental Samples-Paired- Sample Stream Study}

A comparison of the new DAI LC-MS/MS analytical method (schedule 2437) with two existing NWQL analytical methods (schedules 2033 and 2060) for determination of pesticides was conducted in collaboration with the NAWQA Program. Environmental samples, field-matrix spikes, and field blanks collected from a variety of NAWQA stream sites from May to September 2012, as well as routine laboratory QC samples, were analyzed by all three methods. Samples analyzed by the new method were compared to the existing pesticide methods by determination of the common analytes in existing method spike mixtures used as third-party check standards and in environmental samples and field-matrix spikes collected during a NAWQA field study. The results provide a characterization of the performance of the new method during implementation in routine production, including bias and variability of analytes in schedule 2437 over a range of environmental matrices and concentrations and in comparison to schedules 2033 and 2060; potential for bias and contamination using the new field filtration procedure for schedule 2437; and an opportunity to assess efficiency, costs, implementation, and logistical issues of schedule 2437 from both field and laboratory perspectives.

\section{Field-Matrix Spikes}

\section{Study Design}

Environmental samples, spike samples, and spike replicate samples were collected at 48 NAWQA stream sites two to six times during May through September, 2012; most sites had spike samples collected three times and a spike replicate one time. During this study, there were a total of 146 spike samples and 50 spike replicates, resulting in 196 spike samples that used the schedule 2437 spike mixture. In addition, four spike samples and one spike replicate were incorrectly spiked with the schedule 2033 spike mixture, resulting in an expected spike concentration of 4,747 $\mathrm{ng} / \mathrm{L}$, and these are summarized separately.

Recovery of analytes in the spike samples was calculated using equation 3 . Spike-recovery calculation can have large bias and variability if the environmental sample concentration is close to or greater than the spike concentration. This is illustrated in examples of calculated spike recovery for analytes with concentrations of pesticide compounds in the environmental samples larger than the spike level of $250 \mathrm{ng} / \mathrm{L}$ in supporting figure S7. Graphs of spike recoveries relative to environmental sample concentration for all analytes are shown in supporting figure S8. Recoveries for samples with the analytes for which the environmental sample concentrations were larger than the spike level (shown as open symbols in supporting figure S8) generally have larger bias and are not within the data-quality objectives. Consequently, sample results with spike concentration/environmental sample concentrations $>1$ were excluded from the data summary discussed here. There were 36 analytes with background environmental sample concentrations higher than the spike level, although most of these had from 1 to 8 results that were excluded $(<5$ percent of the 146 spike samples). There were seven pesticide compounds (acetochlor OA, metolachlor, metolachlor OA, metolachlor SA, 2,4-D, atrazine, and didealkylatrazine) that had from 9 to 46 results excluded (supporting table $\mathrm{S} 8$ ).

\section{Spike Recovery in Stream Samples}

The results of the calculated spike recovery for the spike samples are shown in table 28. Example graphs of atrazine and atrazine degradates are shown in figure 8 (graphs of matrix-spike recovery by site for all analytes are shown in supporting figure S7). Most analytes had low environmental sample concentrations, so results for all 146 spike samples for each analyte were included in the data analysis. There were 36 analytes with high environmental sample concentrations that were excluded in some samples, so the number of spike samples included in the data analysis was between 
$\boldsymbol{A}$

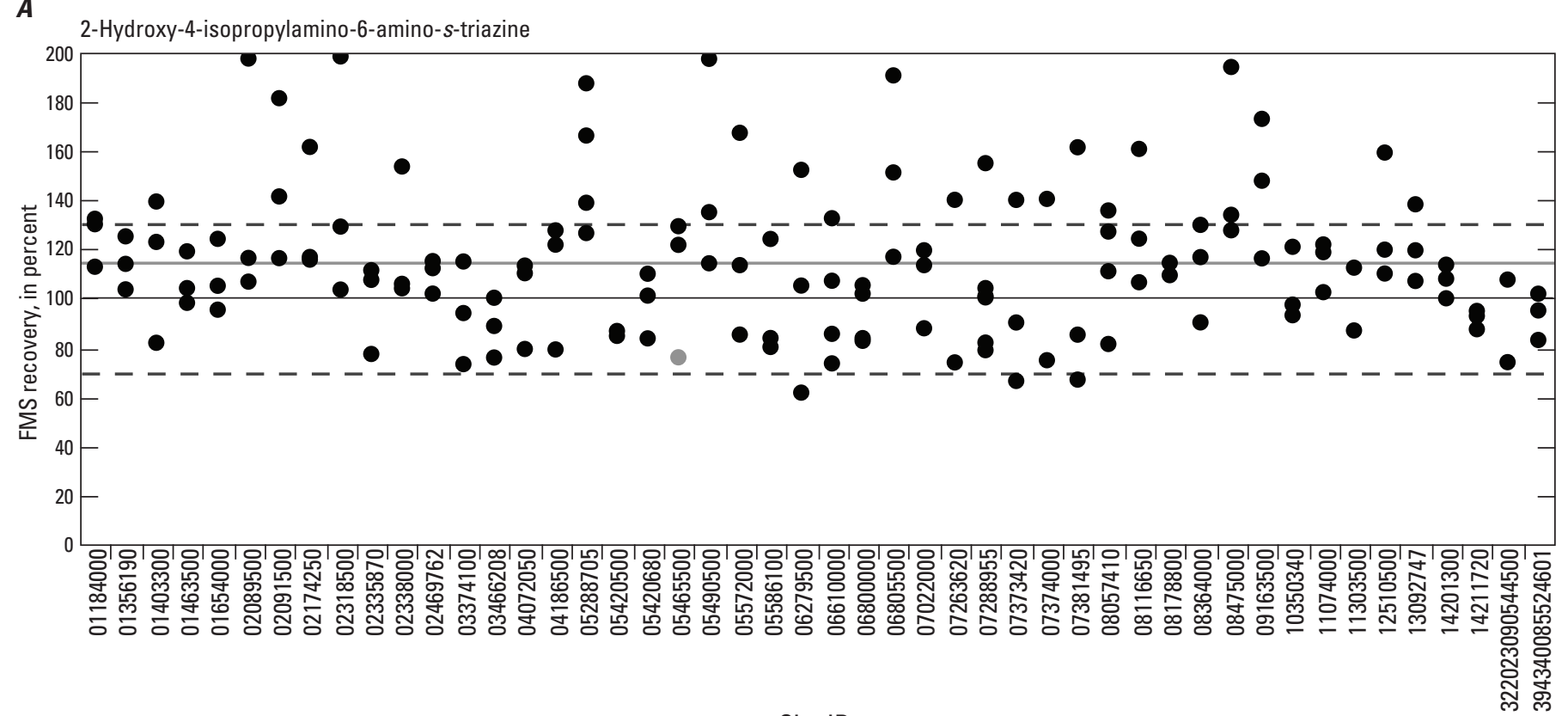

Site ID

B

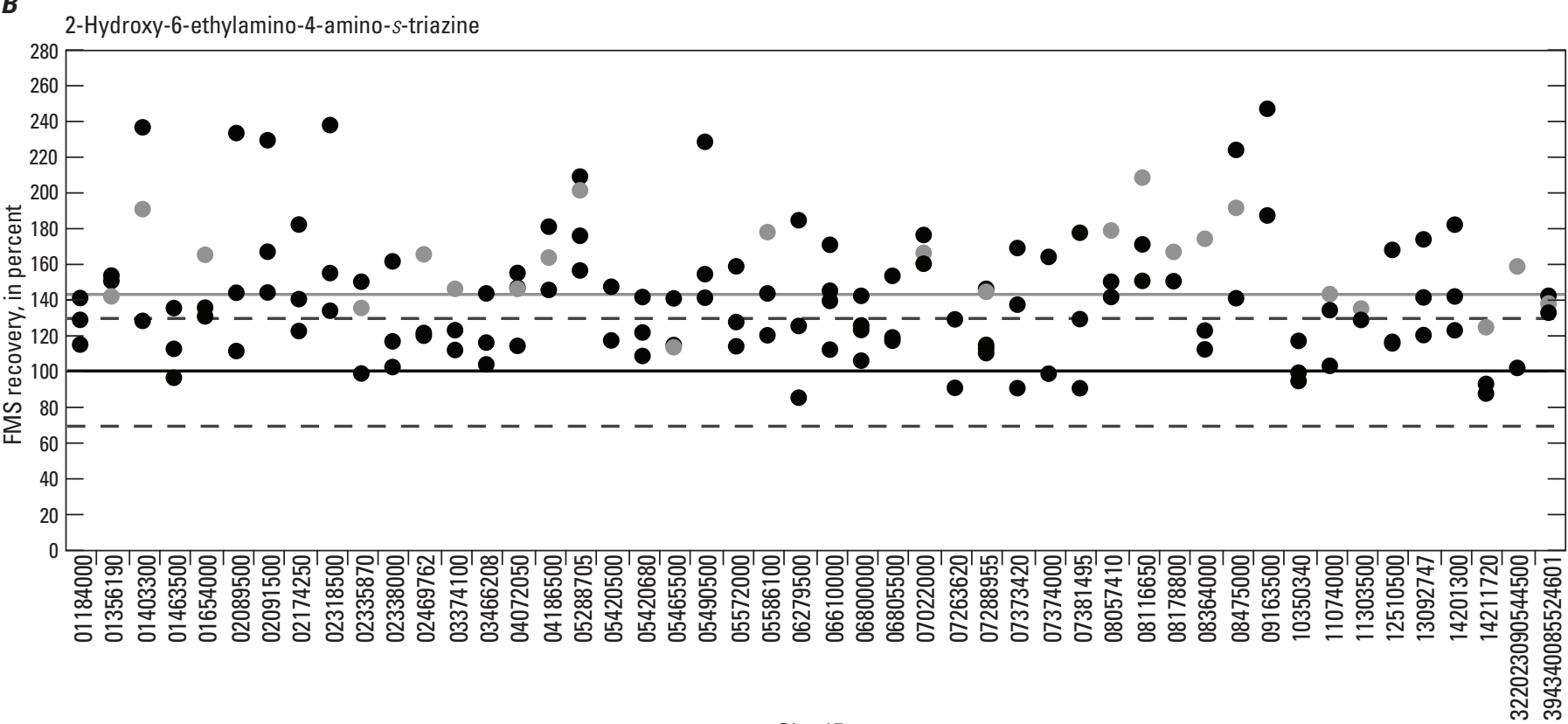

Site ID

Figure 8 (above and following pages). Recovery of atrazine and degradates determined by direct aqueous-injection liquid chromatography-tandem mass spectrometry (DAI LC-MS/MS) in 146 field-matrix-spike samples collected during the National Water Quality Assessment (NAWQA) Program field study from May to September 2012. Recovery of 100 percent shown as solid black line, 70 percent and 130 percent shown as dashed lines; results reported with " $E$ " validation quality code because of interference shown in light gray filled symbols, results with nominal spike level/environmental concentration $<1$ shown as open symbols. (ID, identifier) 
c

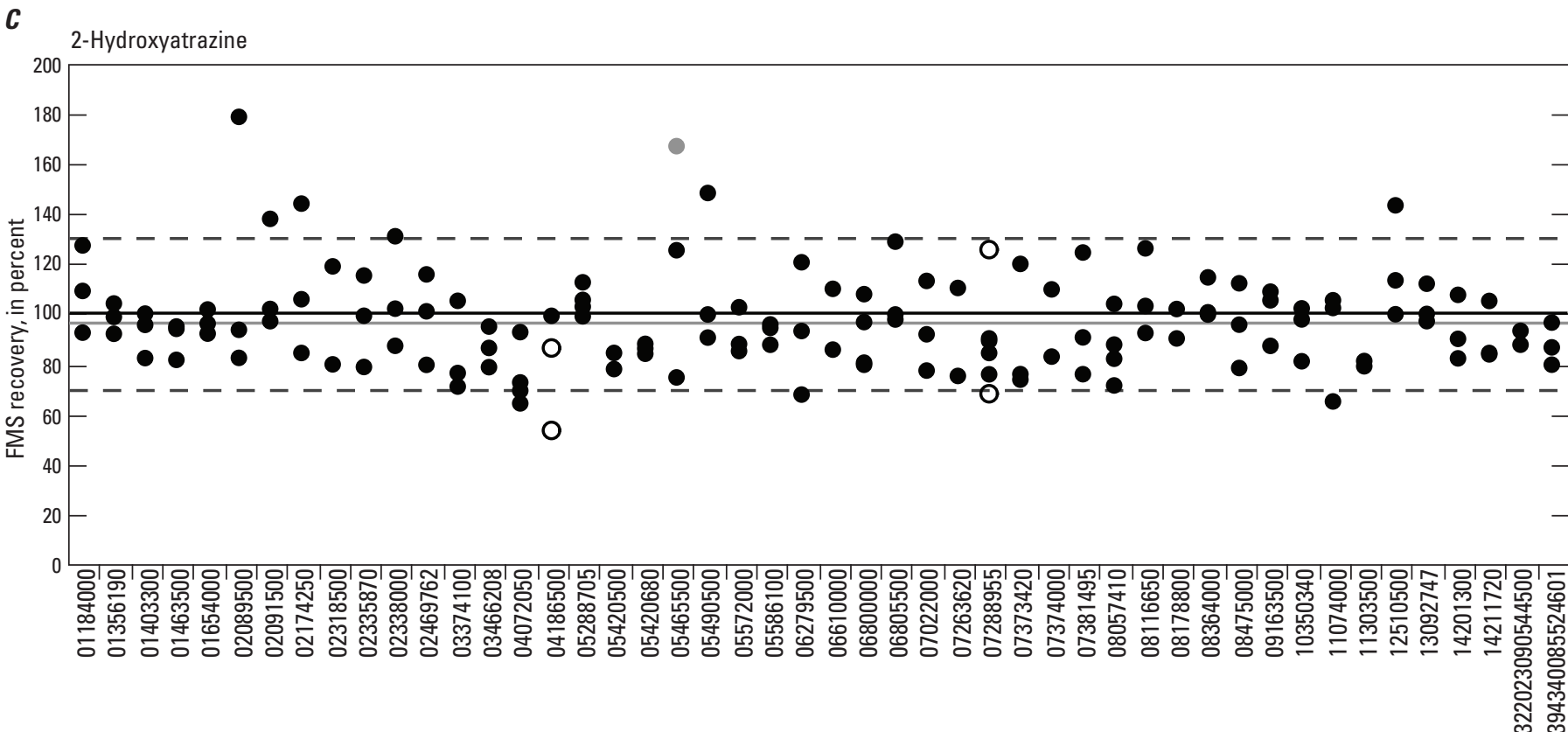

Site ID

D

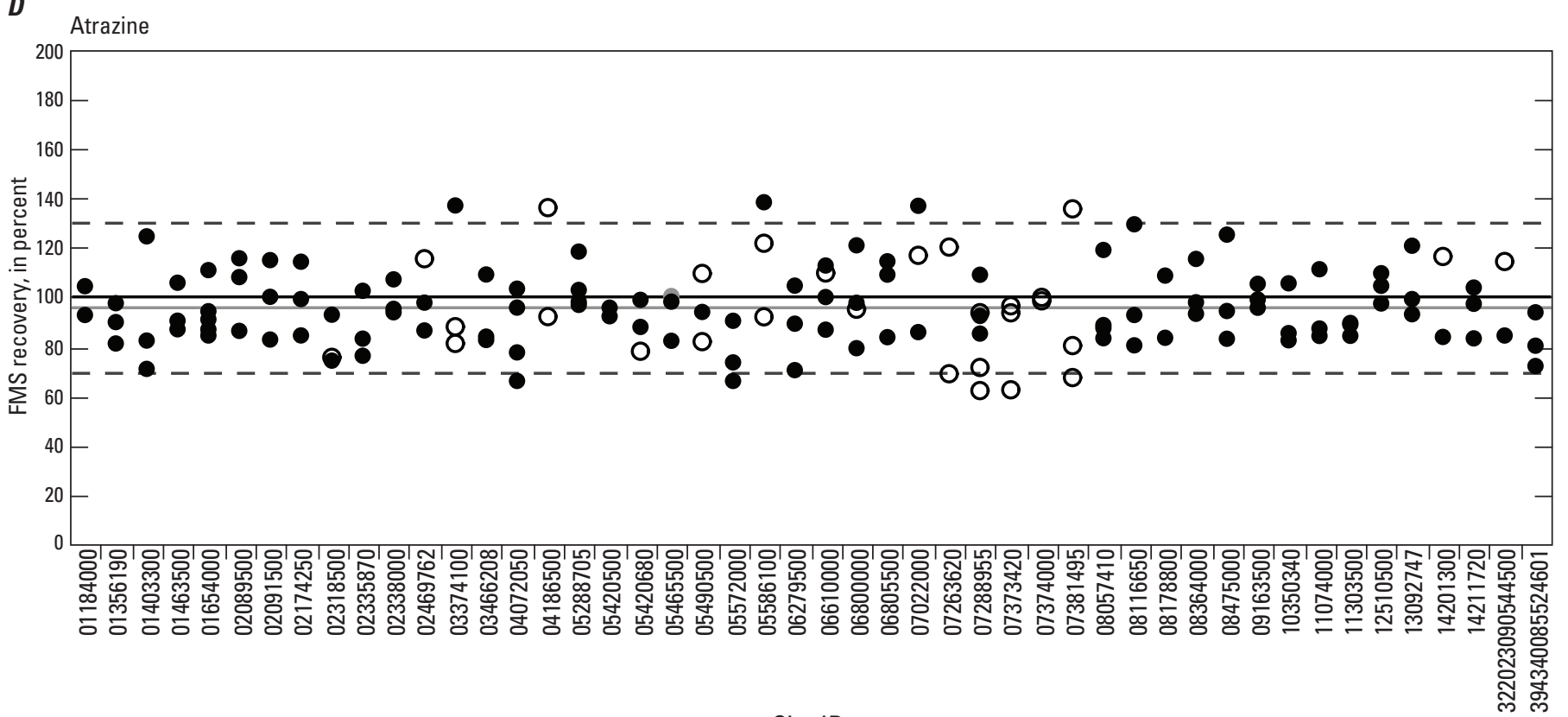




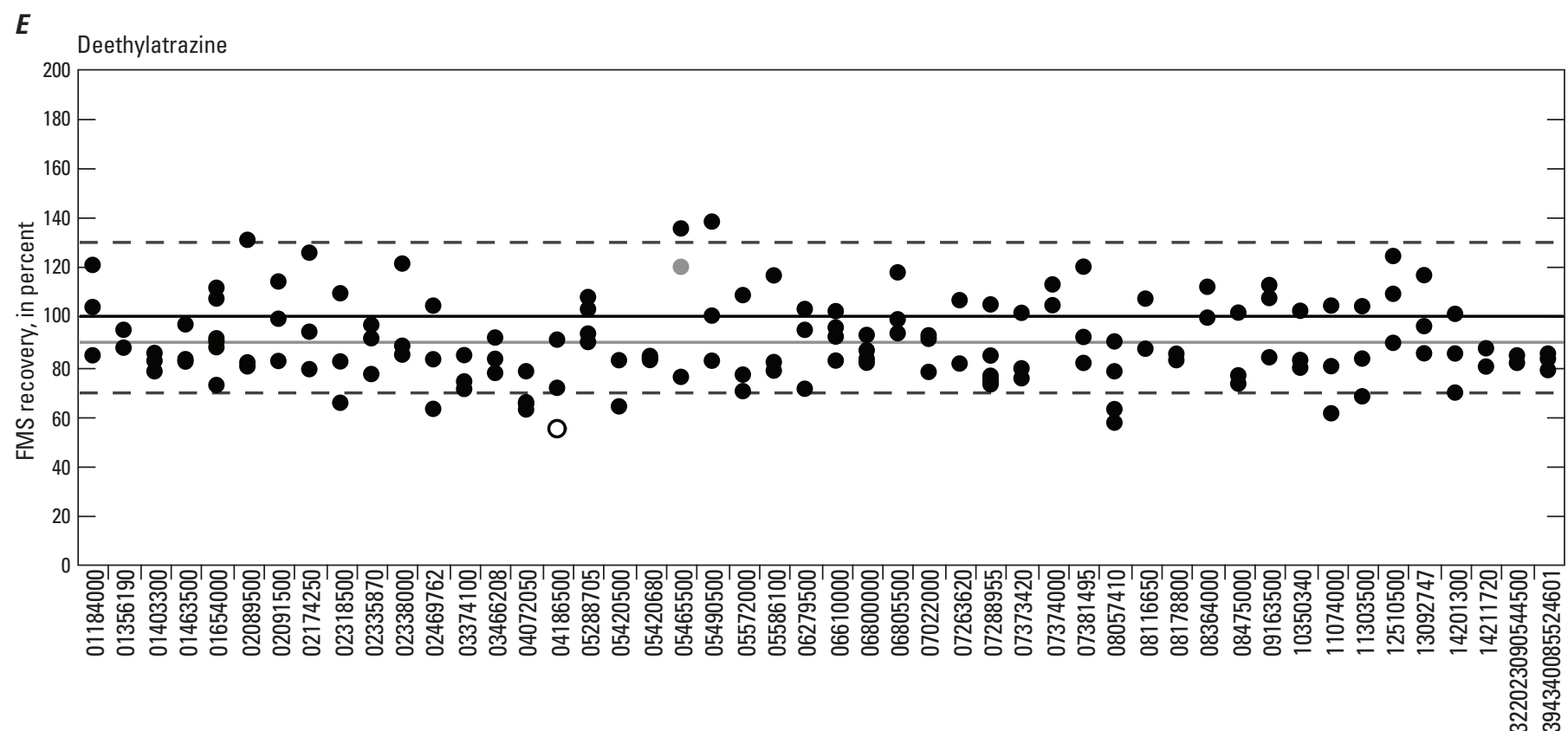

Site ID

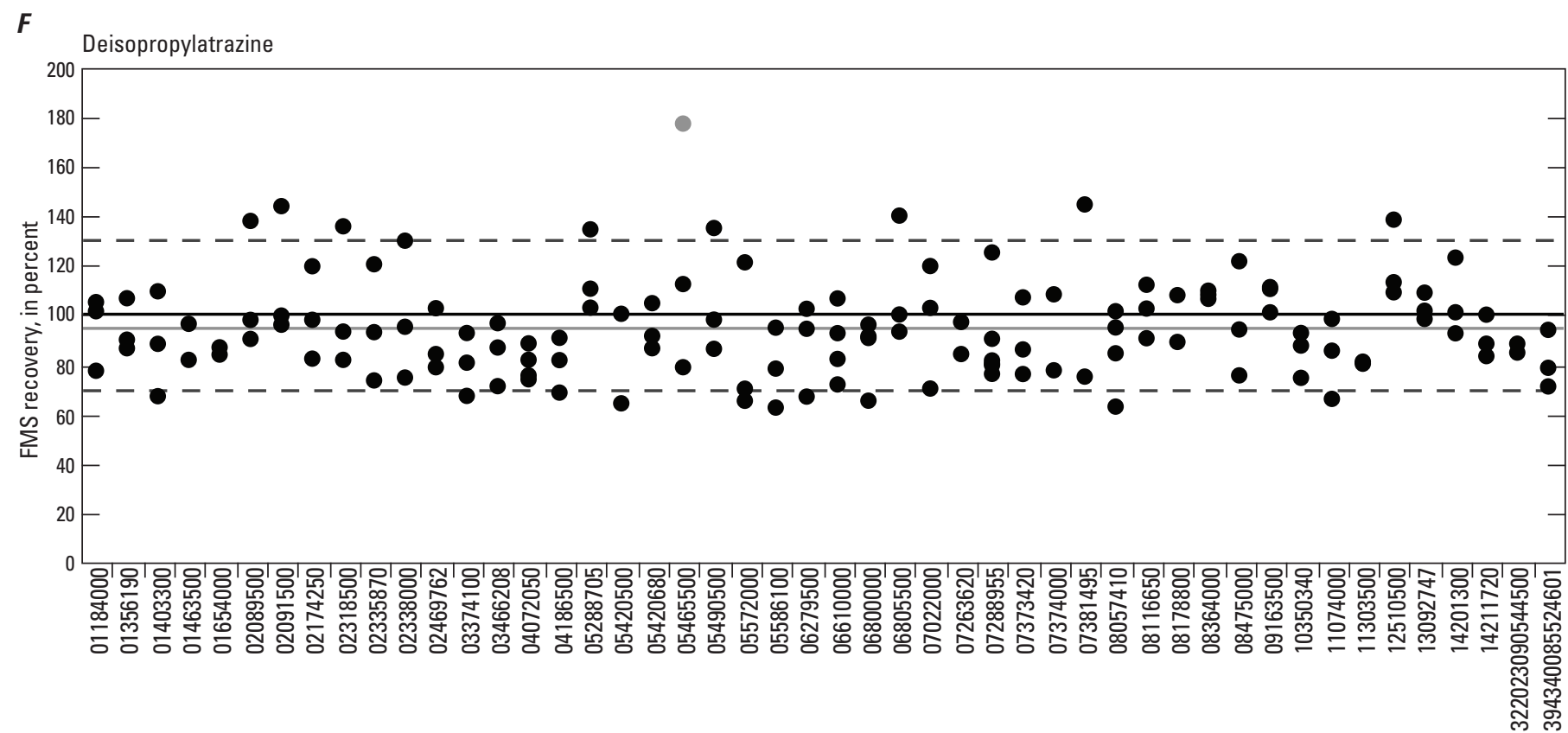

Site ID 
G

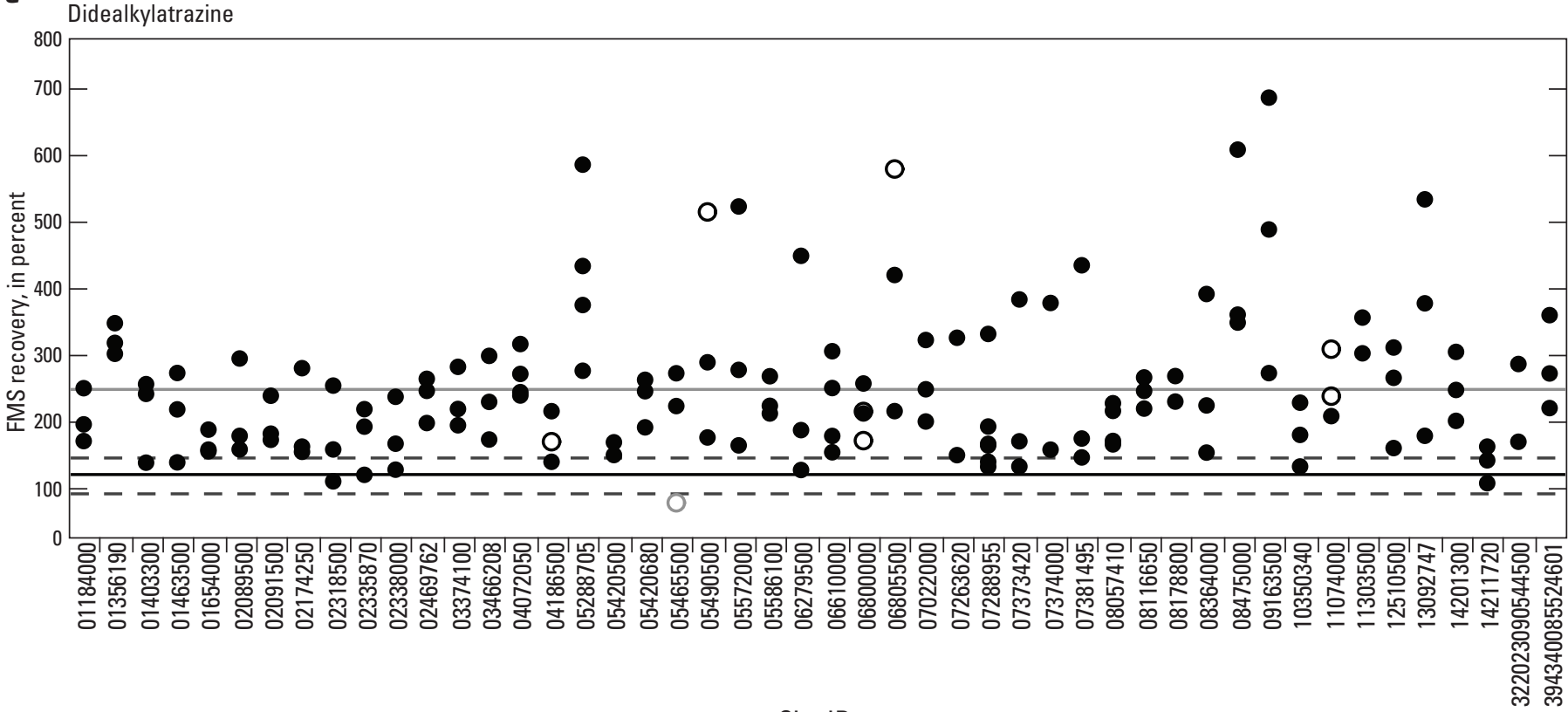

Site ID

H

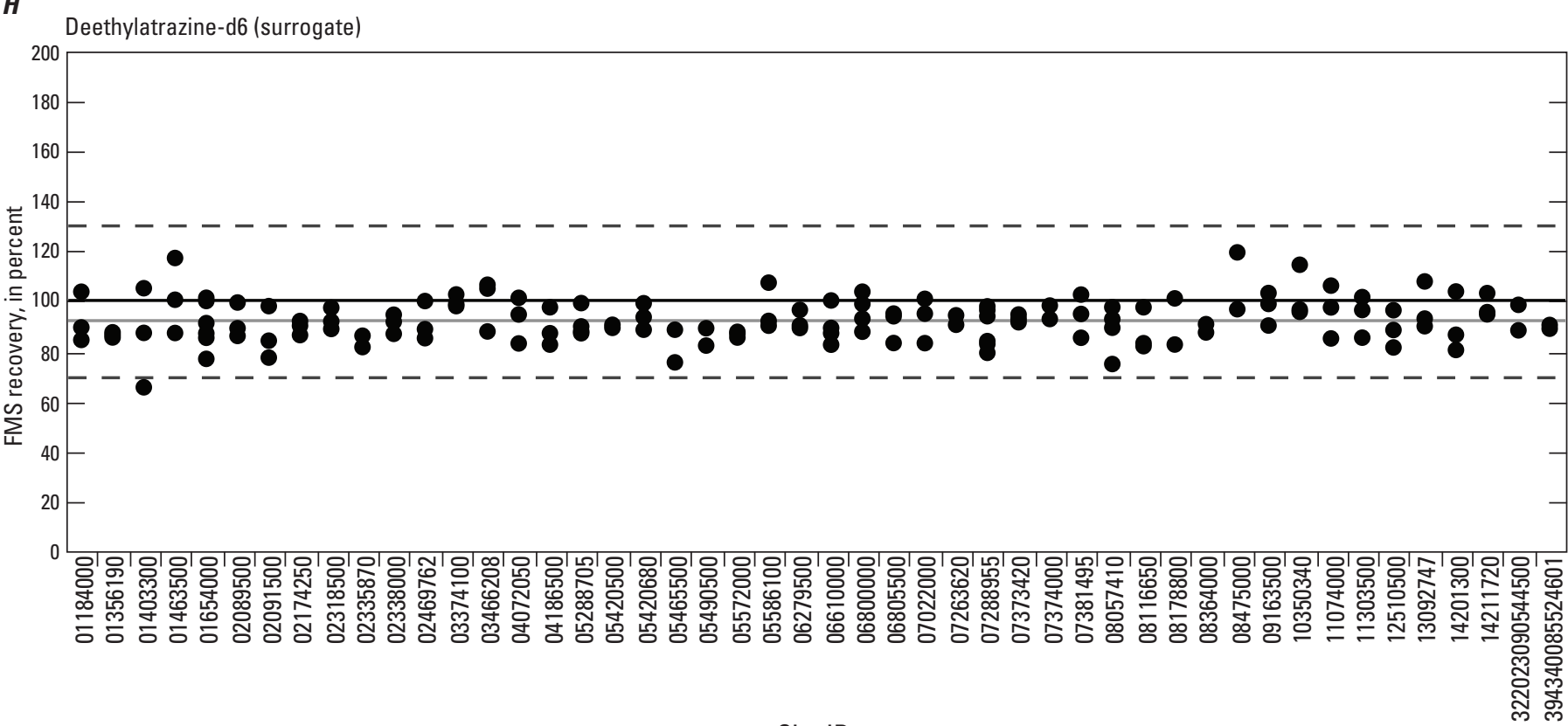

Site ID

Figure 8. Recovery of atrazine and degradates determined by direct aqueous-injection liquid chromatography-tandem mass spectrometry (DAI LC-MS/MS) in 146 field-matrix-spike samples collected during the National Water Quality Assessment (NAWQA) Program field study from May to September 2012. Recovery of 100 percent shown as solid black line, 70 percent and 130 percent shown as dashed lines; results reported with "E" validation quality code because of interference shown in light gray filled symbols, results with nominal spike level/environmental concentration $<1$ shown as open symbols. (ID, identifier)—Continued 
100 (metolachlor SA) and 146. Two pesticide compounds (famoxadone and 1H-1,2,4-triazole) had fewer than 146 matrix-spike sample results because of missing values reported-generally because of interferences. Mean recoveries of most pesticide compounds (192 of 207 pesticide compounds not qualified, labeled " $\mathrm{A}$ " in tables) in field-matrix spikes were within the data-quality objective of $100 \pm 30$ percent, with a median recovery of 93 percent and RSD of 29 percent, although for some stream matrices recoveries were outside these objectives.

For analytes with a validation quality code "A," there were four analytes with mean recoveries higher than 130 percent (diketonitrile isoxaflutole, sulfentrazone, 2-hydroxy-6-ethylamino-4-amino-s-triazine [HDIA], and didealkylatrazine). Didealkylatrazine and HDIA elute in the first few minutes of the chromatogram and have wide and non-Gaussian chromatographic peak shapes, so they are likely influenced by co-eluting stream matrix components that elute in the early part of the chromatogram. Matrix suppression is the likely cause of mean recoveries less than 70 percent for 11 pesticide compounds (oxamyl, indoxacarb, malaoxon, terbufos oxon, chlorimuron-ethyl, hydroxymonodemethyl fluometuron, hydroxyfluometuron, halosulfuron-methyl, nicosulfuron, orthosulfamuron, and hexazinone TP E) in the field-matrix-spike samples. These compounds typically have recoveries within $100 \pm 30$ percent in reagent-water samples. Recoveries were more variable (30 to 54 percent) in the field-matrix-spike samples than study objectives for about 23 pesticide compounds (highlighted in bold in table 28), indicating some matrix effects for these analytes.

Some sites had sample matrices that resulted in low recoveries of some analytes covering a wide range of chemical classes. For example, recoveries of carbaryl, carbofuran, kresoxim-methyl, trifloxystrobin, indoxacarb, isoxaflutole, lactofen, propargite, dimethoate oxon, profenofos, hydroxytebuthiuron, and malathion were between 0 and 50 percent in matrix-spike samples from Iowa River at Wapello, Iowa; Des Moines River at Keosauqua, Iowa; and Platte River at Louisville, Nebr. (supporting figure S7). These sites were characterized by some of the highest $\mathrm{pH}$ values (8.4 to 9.6) of all stream sites, whereas other water-quality parameters such as specific conductivity, major ions, and dissolved organic carbon were not atypical at these sites (supporting tables S9 and S10). Hydrolysis is more rapid for these pesticides at basic $\mathrm{pH}$, which likely resulted in their degradation after they were spiked at the field site and stored at $4{ }^{\circ} \mathrm{C}$ until analysis (about 5-10 days). For example, the half-life of carbaryl is 1.8 days at pH 8 and 10.5 days at pH 7 (Howard, 1991). This degradation of carbaryl over time is in contrast to the surrogate carbaryl$\mathrm{d}_{7}$ which had recoveries within expected limits in these same samples - the surrogate is added to the samples prior to analysis so there is less time for hydrolysis of the surrogate. Recovery of carbaryl in Iowa River at Wapello, Iowa samples with $\mathrm{pH}$ values between 8.8 and 9.2 were 3 to 59 percent, whereas the carbaryl- $\mathrm{d}_{7}$ recovery was from 54 to 102 percent. This is an example of a sample matrix effect that results in biased results, but the matrix effect is not related to ionization suppression in the ion source of the LC-MS/MS instrument. Similar results for degradation of carbamates in high $\mathrm{pH}$ samples were observed during development of EPA method 538, also a DAI LC-MS/MS method for pesticides (Shoemaker, 2011).

One of the disadvantages of a method with a large number of analytes having wide range of chemical characteristics is that it is difficult to find sample preservation techniques that stabilize all analytes without adversely affecting some. Future improvements to the method should explore better sample preservation techniques such as $\mathrm{pH}$ adjustment.

Mean recoveries of most surrogates in the stream samples were generally within the objectives of $100 \pm 30$ percent. Mean recoveries of each surrogate in the stream samples were from 77 to 111 percent, with RSD between 6 and 23 percent (table 28). Recoveries of carbaryl- $\mathrm{d}_{7}$, malathion- $\mathrm{d}_{10}$, and cis-permethrin- ${ }^{13} \mathrm{C}_{6}$ were more variable than the other surrogates, with recoveries from 10 to 175 percent, and RSDs greater than 15 percent (17 to 23 percent). The larger variability of these surrogates indicates they are more susceptible to interference from the sample matrix.

Another 22 analytes shown in table 28 are listed separately and reported with the estimated validation quality code because of difficulty in meeting data-quality objectives during method validation. Also shown are spike recovery results for five analytes that were analyzed by the ESI- mode but had better performance in the ESI+ mode and one analyte that was analyzed by the ESI+ mode but had better performance in the ESI- mode. Recovery results for 10 analytes deleted from the LC-MS/MS method are also shown (analytes with the " $\mathrm{D}$ " validation quality code at the bottom of table 28), illustrating the high bias and variability in these stream samples.

There were samples from five sites that incorrectly used the schedule 2033 spike mixture to prepare the matrix spikes. This mixture is a higher concentration than the schedule 2437 mixture, so the expected spike concentration of the 20 -mL samples was $4,747 \mathrm{ng} / \mathrm{L}$. The results of the calculated spike recovery for these samples are shown in table 29. There were 60 analytes in the 2033 spike mix also in the 2437 analytical method. The average recoveries of most analytes were within the study objectives of $100 \pm 30$ percent. These results show overall good consistency between the two methods, even though the spike mixtures were prepared from different concentrated standard solutions. A few organophosphate analytes (chlorpyrifos oxon, fenamiphos, malaoxon) had lower recovery, although recoveries of these analytes in the same matrices using the schedule 2437 spike mix are acceptable. This might indicate degradation of these pesticide compounds in the 2033 spike mixture ampoule.

\section{Relative Percent Difference of Spike Recovery}

Differences in recoveries of the pesticide compounds in spike and spike-replicate samples provide information on reproducibility of the analysis in a particular sample matrix. There were 50 spike replicate samples collected from 45 sites 
( 3 sites had two pairs of replicate spikes, 1 site had three pairs; 1 site that used the 2033 spike mix was excluded). The difference in recovery was calculated as the relative percent differences (RPD) and summarized in table 30. Spike samples with background environmental concentrations of the pesticide compounds larger than the nominal spike level of $250 \mathrm{ng} / \mathrm{L}$ were analyzed separately. The median RPD of the pesticide compounds with a validation quality indicator of "A" and low background environmental concentration was from 4 to 20 percent for 169 of the ESI+ analytes and from 4 to 28 percent for 38 of the ESI- analytes. Most of the pesticide compounds had a median RPD of less than 15 percent (190 out of 207 of these analytes). Pesticide compounds with a validation quality indicator code of "E" had somewhat larger RPD, from 4 to 39 percent.

\section{Field Blank Samples}

Field blank samples were analyzed from 43 sites during the NAWQA field comparison study. There were few detections of pesticide compounds in field blanks, with only 15 detections found in more than 9,000 determinations (229 pesticide compounds in 43 samples). Twelve analytes were found in at least one of the 43 field blank samples analyzed, with three analytes (pyrimidinol, metolachlor, and methoxyfenozide) detected in two blank samples (table 31). Concentrations were from 1 to $20 \mathrm{ng} / \mathrm{L}$ and close to the LRLs, except for pyrimidinol (a diazinon degradate) where concentrations were 69 and $113 \mathrm{ng} / \mathrm{L}$.

These results indicate relatively low tendency for bias from contamination for analytes in the DAI LC-MS/MS method from all field collection and processing and laboratory analysis steps. Pyrimidinol was an exception, found in two blanks ( 5 percent detection frequency) at relatively high concentrations. This analyte appears to be derived from degradation of the diazinon- $\mathrm{d}_{10}$ internal standard because it has been consistently found in laboratory blanks. Environmental sample results might need to be censored at an appropriate LRL based on summaries of concentrations found in field and laboratory blanks.

The field blank samples with analytes detected were reanalyzed after storage in the freezer for about 3 months to confirm the detections. Six of the analytes found at concentration from 1 to $8 \mathrm{ng} / \mathrm{L}$ were not confirmed in the re-analysis. A likely explanation is that the initial contamination was not in the sample collected at the field site, but introduced at the laboratory during processing and analysis. However, another explanation is that the analytes degraded during storage in the environmental matrix because the holding time studies were done in reagent water. Careful monitoring of laboratory blanks and more experience with the method after implementation in the laboratory might help identify and eliminate the sources of laboratory contamination as a contribution to the field blank contamination.

\section{Environmental Sample Results}

The new DAI LC-MS/MS method expanded the number of pesticide compounds (229) determined in the samples compared to previous methods (about 100 compounds in three different analytical methods). These analytes were rated high-priority for monitoring and studies because of measured or predicted detection frequency, concentration in streams and groundwater, and agricultural and nonagricultural pesticideuse estimates. The results of the initial field comparison study show that a relatively large number of these high-priority pesticide compounds were found at many sites. Mixtures of 3 to 56 pesticide compounds were found in different streams and times, with a median of 26 pesticide compounds for all samples. Mixtures of more than 14 pesticide compounds were found in 75 percent of the samples (25th percentile was 14 detections). The number of detections and summary of concentrations of the pesticide compounds are shown in table 32 .

Seventeen pesticide compounds were found frequently, with detection frequencies from 52 to 86 percent (shown in bold in table 32). The pesticide compounds found most frequently included herbicides heavily used in agriculture, primarily corn and soybean production: atrazine and five degradates (2-hydroxy-4-isopropylamino-6-amino-s-triazine, 2-hydroxyatrazine, deethylatrazine, deisopropylatrazine, and didealkylatrazine), metolachlor and three degradates (dechlorometolachlor, hydroxymetolachlor, and metolachlor SA), and acetochlor SA+alachlor SA mixture. The other pesticide compounds were those used in both agricultural and nonagricultural uses: the fungicides azoxystrobin and carbendazim and herbicides diuron and its degradate (N-(3,4-dichlorophenyl)-N-methylurea), prometon, and the simazine degradate hydroxysimazine. The fungicide carbendazim is a new analyte not previously included in USGS water-quality studies. About 44 percent of the pesticide compounds were not found in any of the samples.

The concentrations of pesticide compounds that were found in the stream samples ranged from undetected to $17,899 \mathrm{ng} / \mathrm{L}$. There were eight pesticide compounds with the median concentration greater than $10 \mathrm{ng} / \mathrm{L}$ (shown in bold in table 32): acetochlor SA + alachlor SA mixture, metolachlor SA, diuron, 2-hydroxyatrazine, atrazine, deethylatrazine, deisopropylatrazine, and didealkylatrazine.

\section{Interlaboratory Comparison of Methods}

An interlaboratory comparison study of stream sample concentrations was conducted with the DAI LC-MS/MS method (schedule 2437) compared to SPE GC-MS SIM method (schedule 2033) and a SPE LC-MS SIM method (schedule 2060). The comparison included linear regression analysis to evaluate potential bias between concentrations determined by the different methods and an evaluation and signed rank test to determine whether there were differences in the number of detections found by the different methods. 


\section{Linear Regression of Concentrations}

For analytes with detections in more than five samples, the agreement between the two methods was evaluated from a linear regression analysis of a graph of the concentrations obtained from each method (Helsel and Hirsch, 2002; Odetokun and others, 2010). A t-test was conducted to determine whether the linear correlation coefficient was different from 0 , and therefore whether a linear correlation exists between the two methods. In addition, if the 95 th percentile of the slope was within 15 percent of unity ( 0.85 to 1.15$)$, the methods were considered similar for the specific analytes. These older methods (SPE GC-MS and SPE LC-MS) involve sample preparation steps that probably introduce more variability than is common for inorganic analytes, where a slope within 10 percent is considered similar (Garbarino and others, 2006).

Graphs of the correlation between concentrations of selected pesticides obtained from schedules 2437 and 2033 are shown in figure 9 and for schedules 2437 and 2060 in figure 10. The graphs show generally good correlations of concentrations determined in the stream samples over 3 to 4 orders of magnitude for pesticides with frequent detections (acetochlor, metolachlor, atrazine, deethylatrazine, simazine, and metalaxyl in schedules 2437 and 2033; 2-hydroxyatrazine, deethylatrazine, atrazine, and 2,4-D in schedules 2437 and 2060). The linear regression analysis parameters are summarized in tables 33 and 34. The linear correlation coefficients were evaluated for pesticide compounds with more than five detections in both methods. These results show that the t-statistic for the linear correlation coefficients (slopes) are significant $(\mathrm{p}<0.001)$ for most of the analytes, and a strong linear correlation exists. The correlation coefficients for imidacloprid in 2437-2060 methods and fipronil sulfide in 2437-2033 methods were not significant, and the graphs show a general lack of correlation of the concentrations (not shown). An explanation for fipronil sulfide lack of correlation is that the concentrations are below the reporting levels of both methods where there is more error in the calculated concentration. Imidacloprid is reported as a highly variable analyte in the 2060 method with large error in the calculated concentrations.

The correlation coefficient for the slope was equal to $1 \pm 0.15$ within the 95 -percent confidence limits for 4 of the 20 compounds in the 2437-2033 comparison (atrazine, metribuzin, prometon, and simazine), indicating that the concentrations were similar and there was low bias between the concentrations. Similarly, the correlation coefficients were equal to $1 \pm 0.15$ for three of the 15 compounds in the 2437-2060 comparison (metalaxyl, 2-hydroxyatrazine, and atrazine). The correlation coefficients for the slope were greater than 1 for the other 16 compounds in the 2437-2033 comparison and generally less than 1 for the other 12 compounds in the 2437-2060 comparison. For example, the slope for acetochlor in the 2437-2033 comparisons was 1.58. This estimate predicts that the concentrations determined by the 2437 method will be 58 percent larger than those determined in the 2033 method. The reasons for these differences might be the result of differences in calibration model (curve), extraction and processing steps, and matrix effects. The 2437 method used spiking solution standards from the 2033 methods as third-party check standards, and the recovery of acetochlor in those standards was 85 percent (table 24). Similarly, the recovery of acetochlor in stream matrix spikes was 96 percent (table 28). These differences would account for 19 percent larger concentrations in the 2033 concentration compared to the 2437 concentration. Other factors, such as matrix enhanced response for the GC-MS method and use of weighting in the 2437 calibration model compared to no weighting in the 2033 method might be responsible for the remaining differences. Additional explanation related to matrix-spike recovery in paired samples determined by the 2033 method are described in the field comparison study.

\section{Detection Frequency in Stream Samples}

The consistency of detection frequency of the different methods was compared by counting the number of detections (at any concentration) and nondetections in the paired-stream samples reported by each method. Graphs of the concentrations found by the different methods (figs. 9 and 10) also show agreement or differences in detections, with different symbols for detections in the methods. The consistency in the number of detections and nondetections in the stream samples is summarized in table 35 for the comparison of schedule 2437 and 2033.

Consistent nondetections are paired samples where the pesticide compound was not detected by both methods and consistent detections are paired samples where the pesticide compound was detected by both methods. Inconsistent detections in one method are paired samples where the pesticide compound was not detected by one method and was detected in the other. Agreement between results reported for both methods as detections or as nondetections was from 66 to 100 percent. For example, the agreement between methods for metalaxyl was 76 percent, with both methods reporting 85 nondetections and both methods reporting 29 consistent detections. The lack of agreement for metalaxyl was 24 percent, with three detections reported as nondetection by schedule 2437 when a detection was reported by schedule 2033, and 33 detections reported as nondetection by schedule 2033 when a detection was reported by schedule 2437 (fig. 9).

The sign test was used to test whether these differences in the number of detections and nondetections found in the paired-stream samples were significant (table 36). Some analytes had significantly larger number of detections in the 2033 method (acetochlor, carbaryl, chlorpyrifos, deethylatrazine, metribuzin, simazine, desulfinylfipronil, desulfinylfipronil amide, and fipronil sulfide), whereas other analytes had significantly larger number of detections reported with the 2437 method (metalaxyl, dicrotophos, dimethoate, tebuthiuron, and hexazinone).

Most of the differences in number of detections were close to or below the LRL of the method, as expected by the definition of MDL and LRL. For example, the detections of acetochlor by the 2033 method that were reported as 
A

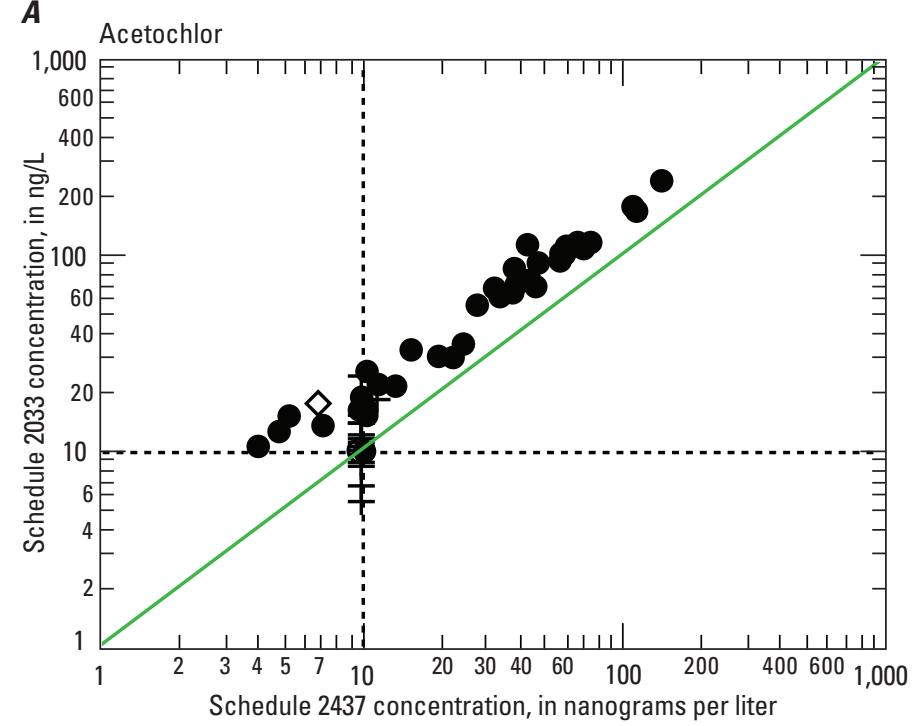

C

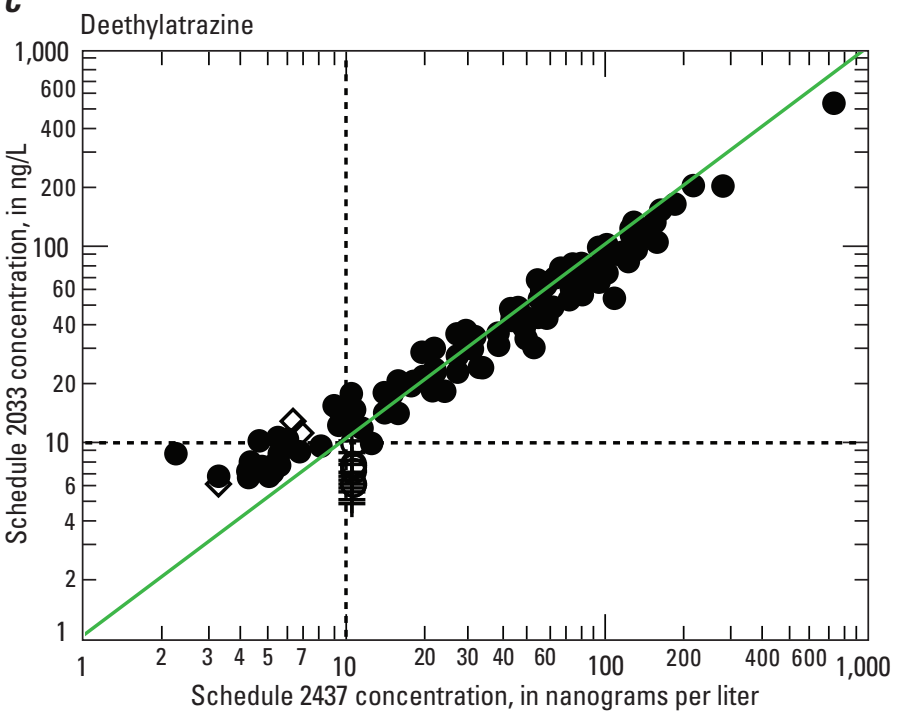

$E$

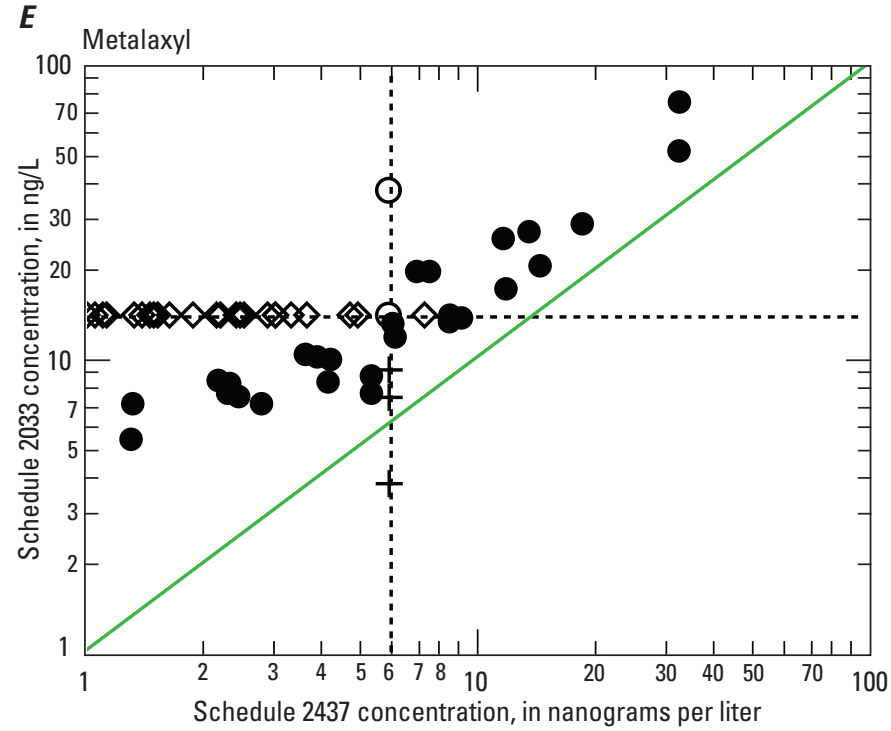

B

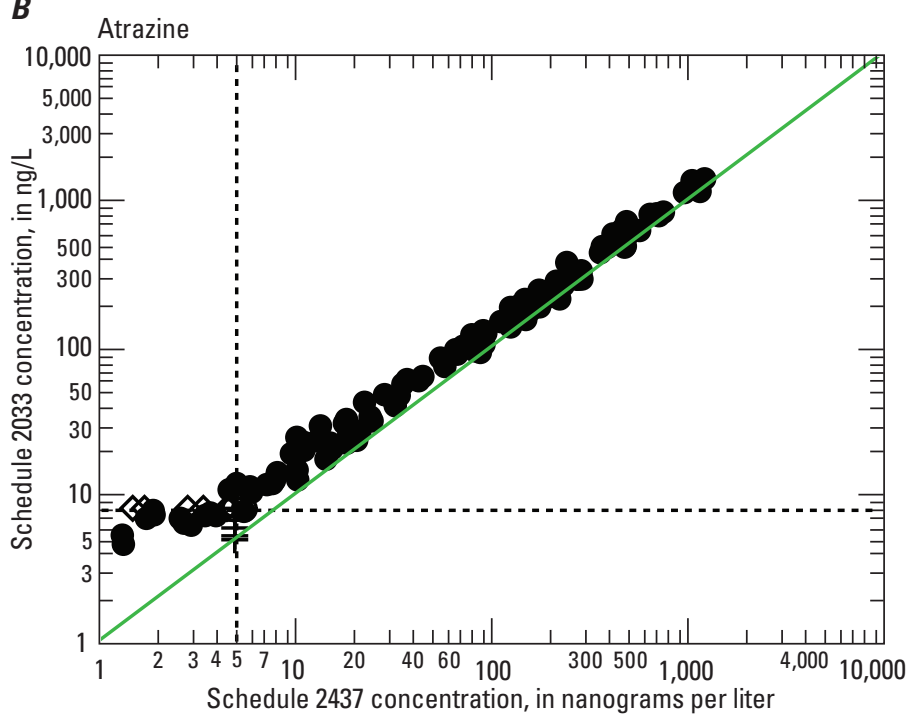

D Metolachlor

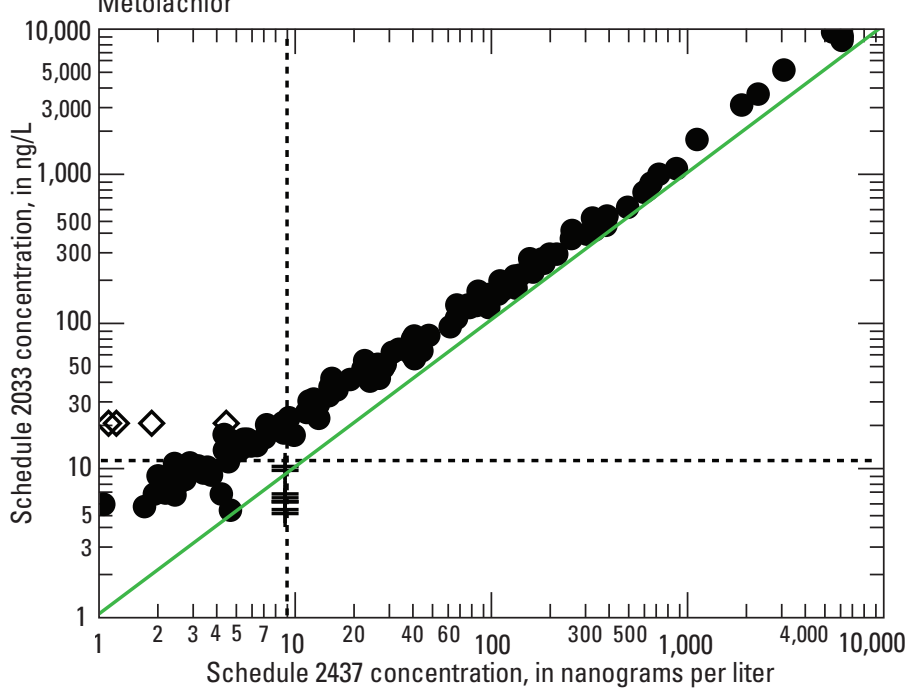

$\boldsymbol{F}$

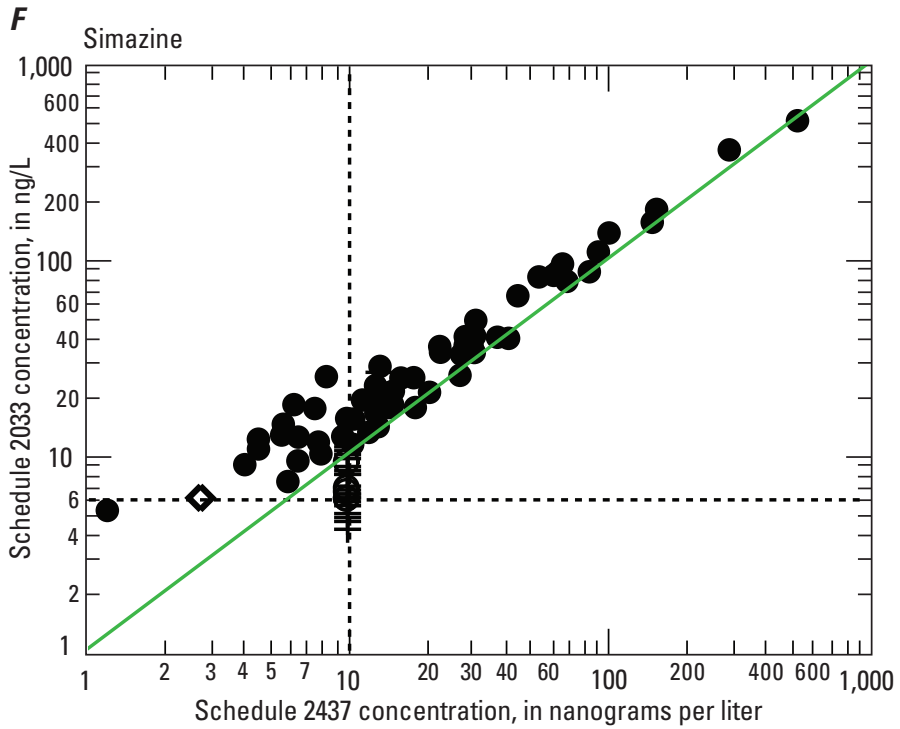


Figure 9 (previous page). Comparison of selected pesticides determined by direct aqueous-injection liquid chromatography-tandem mass spectrometry (DAI LC-MS/MS) (schedule 2437) and gas chromatography-mass spectrometry (GC-MS ) (schedule 2033) in 150 samples from 48 stream sites collected during National Water Quality Assessment (NAWQA) Program field comparison study from May to September 2012. Pesticide compounds detected in both methods shown as filled circles, analyte not detected in either schedule as open circles, analyte detected in 2437 and non-detect in 2033 as open diamonds, and analyte detected in 2033 and non-detect in 2437 as crosses. Laboratory reporting levels in nanograms per liter (ng/L) are shown as dotted lines.

$\boldsymbol{A}$

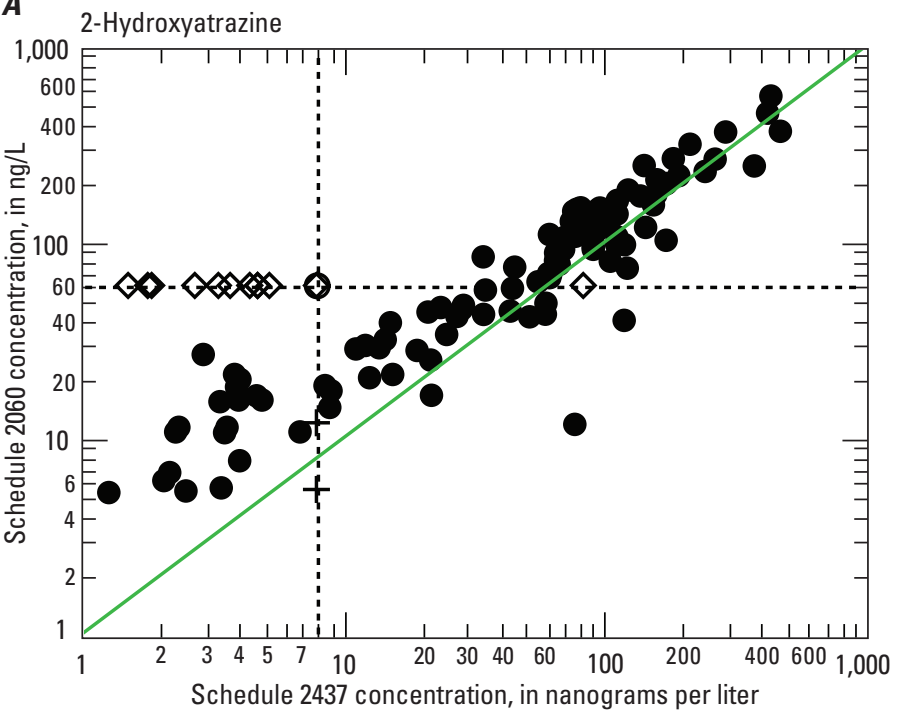

C

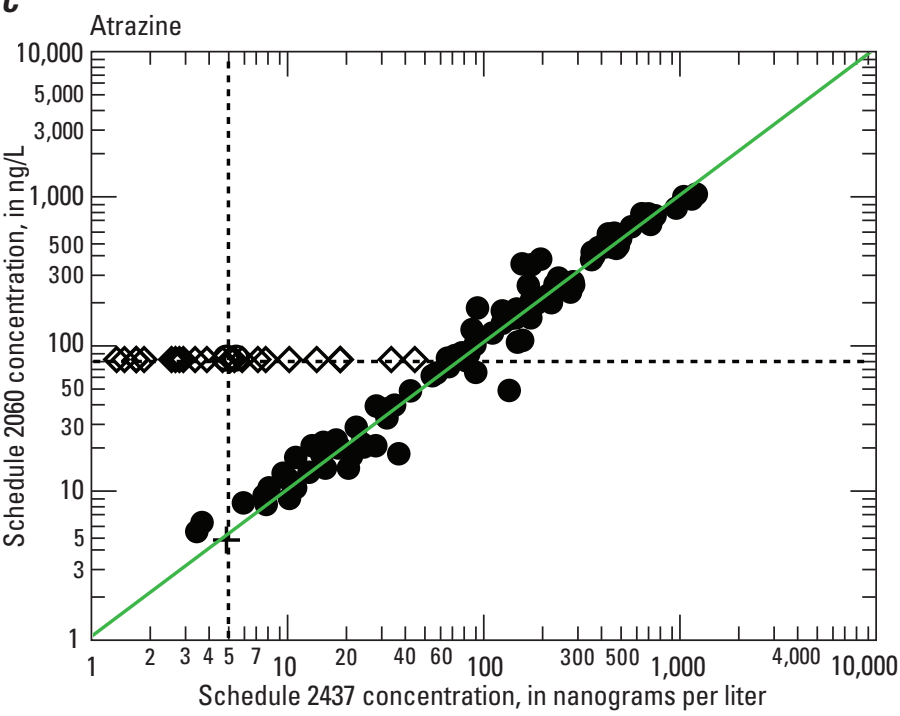

$B$

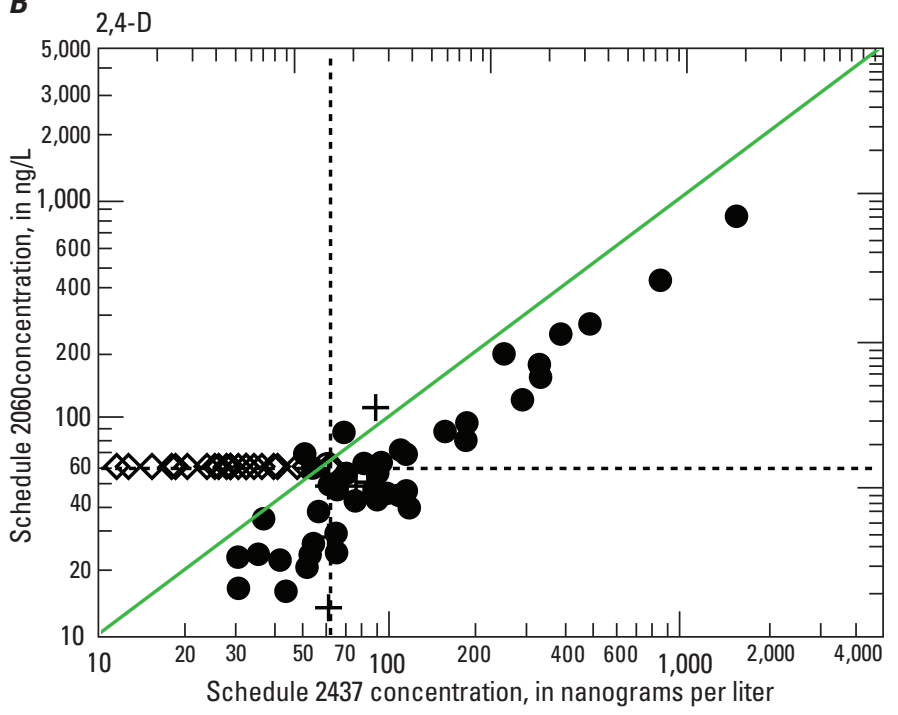

D

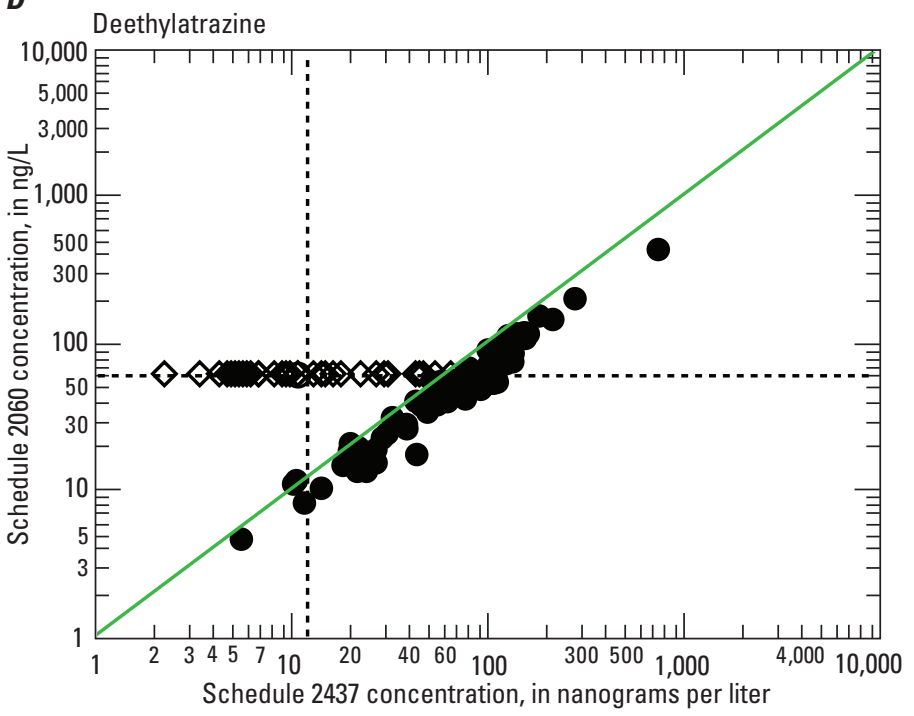

Figure 10. Comparison of selected pesticides determined by direct aqueous-injection liquid chromatography-tandem mass spectrometry (DAI LC-MS/MS) (schedule 2437) and liquid chromatography-mass spectrometry (LC-MS) (schedule 2060) in 150 samples from 48 stream sites collected during National Water Quality Assessment (NAWQA) Program field comparison study from May to September 2012. Pesticide compounds detected in both methods shown as filled circles, analyte not detected in either schedule as open circles, analyte detected in 2437 and non-detect in 2060 as open diamonds, and analyte detected in 2060 and non-detect in 2437 as crosses. Laboratory reporting levels in nanograms per liter (ng/L) are shown as dotted lines. 
nondetections by the 2437 method were reported as concentrations with a median of $10 \mathrm{ng} / \mathrm{L}$ (range from 6 to $24 \mathrm{ng} / \mathrm{L}$ ), similar to the LRL of $10 \mathrm{ng} / \mathrm{L}$ (table 37). Similarly, 90 percent of the 42 detections of simazine in the 2033 method that were reported as nondetections by the 2437 method were less than $11 \mathrm{ng} / \mathrm{L}$, whereas the LRL of the 2437 method was $10 \mathrm{ng} / \mathrm{L}$. On the other hand, 90 percent of the detections of metalaxyl by the 2437 method that were reported as nondetections by the 2033 method were less than $4 \mathrm{ng} / \mathrm{L}$ whereas the reporting level was $14 \mathrm{ng} / \mathrm{L}$ (table 38).

Similarly, the agreement in the number of detections in the stream samples is summarized in table 39 for the comparison of schedule 2437 and 2060. Agreement between results reported for both methods as concentrations or as nondetections was from 56 to 100 percent. For example, the agreement in results in paired-stream samples for diuron was 72 percent, with both methods reporting 86 consistent detections and both methods reporting 21 nondetections. The lack of agreement for diuron was 28 percent, with 4 results reported as nondetection by schedule 2437 when a concentration was reported by schedule 2060, and 37 reported as nondetection by schedule 2060 when a concentration was reported by schedule 2437 .

The sign test was used to test whether there were significant differences in the number of detections found in the paired-stream samples (table 40). Most of the analytes had a significantly larger number of detections in the 2437 method compared to the 2060 method, and most of these detections were lower than the LRL of the 2060 method (tables 41 and 42).

\section{Stability Studies}

Stability of the analytes stored at different temperatures and in potential preservative or dechlorination reagents was measured in different studies. The relevant stability issues for this LC-MS/MS method for pesticides (schedule 2437) include stability in filtered water similar to those expected from time of collection to analysis (refrigeration and ice cooler at $4{ }^{\circ} \mathrm{C}$ ) and storage in a freezer to allow flexibility in sample scheduling. The stability in citric acid ( $\mathrm{pH} 3.8$ ) as a reagent to decrease microbial activity (Pepich and others, 2005) was studied, especially for uses in studies where overnight shipping to the NWQL was unachievable, and stability in ascorbic acid used to reduce free chlorine for studies that collected samples from sites that might have residual chlorine from water treatment plants (Winslow and others, 2001).

\section{Stability Study Design}

Stability of the analytes spiked into reagent water was measured at one concentration under different temperatures and treatments. This design provides information about stability only under the simplest conditions, with minimal microbial activity, stable $\mathrm{pH}$, and low concentrations of dissolved organic carbon and trace metals $(\mathrm{pH}$ and conductivity of the reagent water are shown in table 21). A large study of the effects of various sample matrices is beyond the scope of the validation study. However, this study design will identify analytes that are unstable even in reagent water or with the various preservative treatments.

The recovery of all analytes spiked at a mid-level concentration $(250 \mathrm{ng} / \mathrm{L})$ into reagent water contained in a $20-\mathrm{mL}$ vial was measured after storage at various times for as many as 28 days. Four replicates were prepared for each time interval. Since the LC-MS/MS method includes both pesticide and transformation products (degradates), spike solutions containing only pesticides or pesticide degradates were prepared to conduct the stability studies separately so the transformation product stability can be evaluated independently from the pesticide. At the end of each storage time, subsamples were removed from each vial and analyzed by LC-MS/MS, resulting in five analytical batches.

The four different treatments for pesticides and degradates are shown in table 43 . Note that there was no day zero for the $-10{ }^{\circ} \mathrm{C}$ treatment because the samples took about a day to freeze. Long-term storage of the samples in the freezer was evaluated by re-analysis of a set of samples from day 3 stored in the freezer for 133 days (about 4 months).

Storage stability samples were prepared by adding $100 \mu \mathrm{L}$ of the spike mixtures to $20 \mathrm{~mL}$ of reagent water or treated water in a $40-\mathrm{mL}$ amber vial to exclude light. The treated water samples contained a buffer used in EPA method 527 (reagent water acidified to $\mathrm{pH} 3.8$ with potassium dihydrogen citrate, $9.4 \mathrm{~g} / \mathrm{L}$ ) or a dechlorination reagent (reagent water with ascorbic acid, $0.1 \mathrm{~g} / \mathrm{L}$ ). After adding the spike mixture, the vials were capped and the samples were stored at $4{ }^{\circ} \mathrm{C}$ or $-10{ }^{\circ} \mathrm{C}$ until analysis. At the end of storage, subsamples were transferred to analytical vials and prepared for analysis by adding the internal standard and methanol. Frozen samples were allowed to thaw at room temperature for about $4 \mathrm{~h}$ before subsampling.

All treatments for a particular storage time were analyzed in one analytical batch. Thus, differences over time might reflect batch-to-batch variability as well as any degradation or stabilityrelated changes that occurred during storage. In particular, the samples for ESI+ analytes day 3 were from a repeat experiment, and because these results appear to be higher than other samples, they could give misleading indication of trends over time.

\section{Maximum Holding Times}

Stability results are discussed in terms of recovery rather than concentration. This allows easy comparison of the overall data-quality objectives of recovery within $100 \pm 30$ percent. Stability can then be evaluated as an additional source of bias yielding results lower than 70 percent.

Maximum holding times were estimated using two approaches - a statistical calculation specified by the ASTM and a practical approach based on the data-quality objectives of the validation experiments. The ASTM standard D4841-88 defines the holding time as the time the predicted concentration falls below the lower two-sided 99-percent confidence interval of the analyte concentration found at zero time 
(American Society for Testing and Materials, 1993). The dataquality objectives of the validation experiments include a bias specification within 30 percent of expected concentration or recovery, so the practical approach applied here was changes in recovery lower than 30 percent after storage would indicate the time when degradation of the analyte causes significant bias in recovery.

The 99-percent confidence interval used in the ASTM specification was calculated from $29 \mathrm{CCV}$ standards analyzed during the holding time experiments. The CCVs were analyzed in five separate batches over the 28-day stability study and are expected to provide a good estimate of measurement variability. The tolerable range of variation (99-percent confidence interval) was calculated according to equation 5 :

$$
d= \pm \frac{t \times s}{\sqrt{n}}
$$

where

$$
\begin{gathered}
d \quad \text { is the range of tolerable variation from the } \\
\text { initial mean recovery, in percent; } \\
t \quad \text { is the Student's t (based on number of } \\
\text { replicates); } \\
s \quad \text { is the standard deviation of replicates, in } \\
\text { percent; and } \\
n \quad \text { is the number of replicates at each time. }
\end{gathered}
$$

For this study, $\mathrm{n}=29$ (replicate CCVs) and the Student's $\mathrm{t}$ for a two-tailed 99-percent confidence interval is 2.763. The lower limit of stability was then calculated as 100 percent minus the tolerable range of variation. The practical approach defined the lower limit of stability as 100 percent minus 30 percent ( 70 percent).

\section{Stability Study Results}

Results of the stability experiments by treatment for the two analyte groups (pesticides and degradates) are discussed in the following sections. Storage at $4{ }^{\circ} \mathrm{C}$ and $-10{ }^{\circ} \mathrm{C}$ with no preservative are discussed in detail, whereas stability results with ascorbic acid and $\mathrm{pH} 3.8$ are provided in supplementary tables. The pesticide group is discussed first, followed by pesticide degradates. Analytes determined by ESI+ mode are discussed before the ESI- analytes. Graphs of the recoveries as a function of storage time in the different treatments for each pesticide compound are shown in supporting information figures S9, S10, S11, S12, S13, and S14. Variability of study results is represented by the RSD in tables 44-62.

\section{Tolerable Range of Variation}

The lower limits of stability calculated from the CCVs for analytes determined in the ESI+ mode are summarized in table 44. Most analytes determined by ESI+ mode had relatively narrow tolerable ranges of variation from the mean concentration (99-percent confidence intervals) less than 20 percent (mean, 17 percent; median, 17 percent; 75th percentile, 20 percent). The corresponding lower limits of recovery expected from analytical variability for most analytes were from 80 to 91 percent and were higher than the practical data-quality objectives of recovery of 70 percent. A few analytes had larger confidence intervals, including didealkylatrazine (42 percent), 2-hydroxy-6-ethylamino-4-aminos-triazine (31 percent), and 4-chlorobenzylmethyl sulfoxide (36 percent). The analytes with validation quality code "E" generally had a larger range of confidence intervals, from 13 to 78 percent.

The lower limits of stability calculated from the CCVs for analytes determined in the ESI- mode are summarized in table 45. Most analytes (37 of 45) determined by ESI- mode had tolerable ranges of variation from the mean concentration (99-percent confidence intervals) of 40 percent or less (mean 30 percent; median 25 percent; 75th percentile 31 percent). The corresponding lower limits of recovery expected from analytical variability alone for most analytes were from 63 to 88 percent. Seven analytes (flubendiamide, oryzalin, diflufenzopyr, sulfentrazone, chlorothal-monomethyl, chlorosulfonamide acid, and hexazinone TP D) had larger analytical variability of the CCV samples, so the corresponding lower limits of recovery are very low ( 25 to 52 percent) and might not be useful for estimating holding times.

\section{Stability at $4{ }^{\circ} \mathrm{C}$ Pesticides ESI+ Mode Analytes}

The variability of recoveries, represented by the RSD, of pesticides analyzed in ESI+ mode generally were less than 15 percent for any day, with median of recovery variability from 4 to 9 percent for the five study holding times are shown in table 46 (for the 90 pesticides with validation quality code of "A"). These results indicate that relative measurement error for any storage time was low so that the concentration trends over time were less likely to be obscured by variability in results. Some of the qualified analytes, with a validation quality code of "E" had variability larger than 15 percent, for example oxyfluorfen on day 7 (47 percent) and parathion methyl on day 14 (78 percent). Concentration trends over time for these pesticides will be more difficult to determine.

Changes in recovery of pesticides determined by ESI + mode after 28 days of storage at $4{ }^{\circ} \mathrm{C}$ were relatively low (table 46). Acetanilide, carbamate, fungicide, sulfonylurea, and triazine pesticides were within the ASTM tolerable range of variation with few exceptions. Triallate (79 percent) and kresoxim-methyl (84 percent) exceeded the ASTM limit, but these were above the practical limit of 70 percent. Miscellaneous, organophosphate, and pyrethroid pesticides had slightly larger changes in recovery after 28 days, with many (27) analytes below the ASTM limit after this time. Three organophosphates (disulfoton, phorate, and terbufos) had recoveries lower than 70 percent (54 to 66 percent) after 28 days. In addition, five pesticides (etoxazole, indoxacarb, lactofen, cis-permethrin, and trans-permethrin).were less than the practical limit of 70 percent after 28 days, but they were also below 70 percent on day 0 and other times, so 
any changes in variability might be obscured by the analytical variability. Famoxadone, naled, and phosmet are qualified (E) for various reasons but also appeared to degrade and have recoveries less than 70 percent after storage for 28 days. Overall, all 102 pesticides analyzed in ESI+ mode were 70 percent or above after 14 days of storage, and 92 pesticides were above 70 percent after 28 days of storage. Flumiclorac-pentyl and bifenazate exceeded practical recovery limits after storage for 7 and 14 days, so were considered unsuitable for the method and candidates for deletion after the validation studies.

Few of the targeted degradates were found in the samples spiked with pesticides only and stored at $4{ }^{\circ} \mathrm{C}$, indicating stability of most of the 44 pesticides that have a degradate measured in the ESI+ mode. Fenamiphos sulfoxide, the diazinon degradate pyrimidinol, and the ethoprop degradate O-ethyl-O-methyl-S-propyl phosphorothioate were found at concentrations representing about 5 to 8 percent of the nominal concentration of the pesticide and remained at that level during storage. This might reflect some degradate present in the mixed standard rather than formation during storage. Disulfoton sulfoxide, however, increased from 8 percent on day 0 to 19 percent on day 28 of the spike concentration of disulfoton added to the sample on day 0 and probably represents transformation of disulfoton during storage. Similarly, bifenazate diazine was found at concentrations representing 16 to 70 percent of bifenazate concentration, although both these analytes had very high measurement variability.

\section{Stability at $4{ }^{\circ} \mathrm{C}$ Pesticides ESI- Mode Analytes}

The variability of pesticides analyzed in ESI- mode stored at $4{ }^{\circ} \mathrm{C}$ for as many as 28 days were generally less than 15 percent for most pesticides, although on some days variability was as high as 28 percent for some pesticides. The overall variability was from 6 to 33 percent, with the sulfonylureas diflufenzopyr and sulfentrazone generally having larger variability, as shown in table 47 . There were relatively small changes in recovery of the pesticides over the 28-day storage time. All pesticides were within both the ATSM tolerable range of variation and the practical limit of greater than 70 percent recovery after 28 days except for flubendiamide. Recovery of flubendiamide decreased to 68 percent by day 28. This pesticide had a very low ASTM limit (25 percent) because of the variability of CCVs used to calculate the standard deviation. This probably represents some degradation of flubendiamide in the CCVs during data acquisition of the sample batch. There were few degradates found in the samples spiked with pesticides only and stored at $4{ }^{\circ} \mathrm{C}$, indicating stability of most pesticides (at least those that have a degradate determined by this method). Diketonitrile isoxaflutole was the only degradate found in the pesticideonly spike samples, at 5 and 7 percent of the spike level at day 14 and 28.

\section{Stability at $4{ }^{\circ} \mathrm{C}$ Degradates ESI+ Mode Analytes}

The variability of recoveries of degradates analyzed in ESI+ mode generally were less than 20 percent for any day, with median recovery variability from 5 to 7 percent for the five sampling days (table 48). Chlorpyrifos oxon on day 3 and methomyl oxime and hydroxy monomethyl fluometuron on day 28 were more variable (25-50 percent). 7-Hydroxycarbofuran had high measurement variability ( 24 to 70 percent) on some days.

Changes in recovery of degradates determined by ESI+ mode after 28 days of storage at $4{ }^{\circ} \mathrm{C}$ were relatively low. Overall, 82 of 84 degradates with validation quality code of "A" were above 70 percent recovery after 14 days of storage, and 74 degradates were above 70 percent recovery after 28 days of storage. Many degradates were within the ASTM tolerable range of variation after 14 days of storage but exceeded the limit after 28 days. However, only 10 degradates exceeded the practical limit of 70 percent (phorate oxon, terbufos oxon, 4-hydroxy-tert-fluometuron, hydroxy mono demethyl fluometuron, hydroxyfluometuron, tebuthiuron TP 106, 2-hydroxy-4-isopropylamino-6-aminos-triazine, 2-hydroxy-6-ethylamino-4-amino-s-triazine, didealkylatrazine, and hexazinone TP C), and all were within the practical limit after 14 days of storage except for phorate oxon and terbufos oxon, and for diazinon oxon after 7 days. Methomyl oxime and desiodoflubendiamide were less than 70 percent on day 0 but were above 80 percent after that from 3 to 28 days.

\section{Stability at $4{ }^{\circ} \mathrm{C}$ Degradates ESI- Mode Analytes}

The variability of recoveries of degradates analyzed in ESI- mode were less than 20 percent for day 3 through 28 for about half of the analytes (table 49). Many of the analytes had relatively high recovery (greater than 130 percent) and high analytical variability, from 20 to 30 percent on different days. Despite this high recovery, the overall changes in recovery after 28 days of storage were relatively low. All degradates determined in ESI- mode were within the ASTM tolerable range of variation after 28 days of storage. The ASTM limit was lower than the practical limit of 70 percent for some analytes (4-(hydroxymethyl)pendimethalin, cis-cyhalothric acid, metribuzin DK) because of the larger variability on a particular day. However, the recoveries on day 28 for these analytes were still above the practical limit of 70 percent.

\section{Stability at $-10^{\circ} \mathrm{C}$ Pesticides ESI+ Mode Analytes}

The variability of recoveries of pesticides analyzed in $\mathrm{ESI}+$ mode stored at $-10^{\circ} \mathrm{C}$ were mostly less than 15 percent for any time period, with the median of recovery variability for 91 pesticides from 5 to 9 percent for the five sample storage times (table 50). As in the case for storage at $4{ }^{\circ} \mathrm{C}$ the relative analytical variability for any storage time was low so that the concentration trends over time are distinct. However, recoveries for many analytes were relatively high on day 3 . 
Changes in recovery of pesticides determined by ESI+ mode after 28 days of storage at $-10{ }^{\circ} \mathrm{C}$ were relatively low (table 50) and are similar to the results of storage at $4^{\circ} \mathrm{C}$. Overall, most (101 of 102) pesticides analyzed in ESI+ mode were greater than 70 percent recovery after 14 days of storage, and 94 pesticides were within 70 percent after 28 days of storage. Acetanilide, carbamate, fungicide, sulfonylurea, and triazine pesticides were within the ASTM tolerable range of variation with few exceptions. Triallate ( 83 percent), linuron (88 percent), and siduron (88 percent) exceeded the ASTM limit but were within the practical limit of above 70 percent recovery. Miscellaneous, organophosphate, and pyrethroid pesticides had the larger changes in recovery after 28 days, with many analytes below the ASTM limit after this time. Only eight pesticides were less than the practical limit of above 70 percent recovery (etoxazole, indoxacarb, lactofen, pyridaben, phosmet, oxyfluorfen, cis-permethrin, and trans-permethrin). Notably phorate and terbufos had higher recoveries ( 74 and 86 percent) after 28 days of storage at $-10{ }^{\circ} \mathrm{C}$ compared to $4{ }^{\circ} \mathrm{C}$ (59 and 54 percent). Similarly, flumiclorac-pentyl and bifenazate had higher recoveries (82 and 80 percent) after 14 days of storage at $-10{ }^{\circ} \mathrm{C}$ compared to $4{ }^{\circ} \mathrm{C}$ (21 and 46 percent). Recoveries of pesticides in samples stored at $-10^{\circ} \mathrm{C}$ for 133 days (about 4 months) were similar to those at 28 days, with eight pesticides with recoveries less than 70 percent (etoxazole, lactofen, pyridaben, disulfoton, tribufos, phosmet, cis-permethrin, and trans-permethrin).

The few degradates found in the samples spiked with pesticides only and stored at $-10{ }^{\circ} \mathrm{C}$ were the same ones as found in samples stored at $4{ }^{\circ} \mathrm{C}$. Fenamiphos sulfoxide, pyrimidinol, and O-ethyl-O-methyl-S-propyl phosphorothioate were found at concentrations representing about 5 to 8 percent of the spiked concentration of the pesticide $(250 \mathrm{ng} / \mathrm{L})$ and remained at that level during storage. It is also possible these degradates were the result of contamination, carryover, or some other artifact and not due to degradation of the pesticide because the concentrations of the degradate did not increase over time, and the corresponding pesticide did not decrease. Disulfoton sulfoxide increased from 9 to 20 percent over the 28-day storage, very similar to the samples stored at $4{ }^{\circ} \mathrm{C}$, corresponding to the decrease in disulfoton. This pesticide compound increased during storage at $-10{ }^{\circ} \mathrm{C}$ for 133 days, as well as three other organophosphate degradates (fenamiphos sulfoxide, phorate sulfoxide, and terbufos sulfoxide) (supporting table S12).

\section{Stability at $-10^{\circ} \mathrm{C}$ Pesticides ESI- Mode Analytes}

Storage temperature had no measurable effect on recovery of pesticides determined by ESI- mode. Changes in recovery of pesticides determined by ESI- mode after 28 days of storage at $-10{ }^{\circ} \mathrm{C}$ were relatively low (table 51) and are similar to the results of storage at $4{ }^{\circ} \mathrm{C}$. As in the case of samples stored at $4{ }^{\circ} \mathrm{C}$, all pesticides were within both the ATSM tolerable range of variation and the practical limit of 70 percent recovery after 28 days except for flubendiamide. However, many recoveries were high ( $>130$ percent) on the first two sample storage times, so the fact that recoveries were above 70 percent after 14 and 28 days could also reflect some degradation that was obscured by the high recoveries. Flubendiamide recovery was below 70 percent (69 percent) after 28 days, but 95 percent after 133 days, perhaps a result of batch-to-batch analytical variability of this analyte rather than degradation during storage. Similar to the samples stored at $4{ }^{\circ} \mathrm{C}$, diketonitrile isoxaflutole increased to 13 percent of the spiked concentration during the 28-day storage from degradation of isoxaflutole. Isoxaflutole, a pesticide determined in the ESI+ mode, recoveries correspondingly decreased by 21 percent over the same time. This was also the only ESI- mode degradate found in the samples spiked with pesticides only during storage at $-10{ }^{\circ} \mathrm{C}$ for 133 days (supporting table S12).

\section{Stability at $-10^{\circ} \mathrm{C}$ Degradates ESI+ Mode Analytes}

There were relatively small changes in recovery of degradates determined by ESI+ mode after 28 days of storage at $-10^{\circ} \mathrm{C}$ (table 52). Many acetanilide, carbamate, fungicide, miscellaneous, and organophosphate degradates were within the ASTM tolerable range after 28 days. There were a few exceptions, including 4-hydroxymolinate, carboxy molinate, and desiodo flubendiamide (77, 77, and 78 percent, respectively), but these were above the practical limit of 70 percent recovery. Recoveries were less than 70 percent for only two degradates (diazinon oxon and disulfoton oxon) after 133 days of storage at $-10{ }^{\circ} \mathrm{C}$. Many sulfonylurea and triazine degradates exceeded the ASTM tolerable range at 28 days, and seven were less than 70 percent (4-hydroxy-tert-fluometuron, 2-hydroxy-4-isopropylamino-6-amino-s-triazine, 2-hydroxy-6-ethylamino-4-amino-striazine, didealkylatrazine, 4-hydroxyhexazinone A, hexazinone TP C, and hydroxysimazine). However, these lower recoveries in the samples stored for 28 days might have been the result of bias with that particular analytical batch rather than degradation during storage because the samples stored for 133 days had recoveries above 70 percent. Compared with storage at $4{ }^{\circ} \mathrm{C}$, storage at $-10^{\circ} \mathrm{C}$ substantially improved the recovery of phorate oxon and terbufos oxon, from 39 and 7 percent to 102 and 82 percent recovery after 28 days.

\section{Stability at $-10^{\circ} \mathrm{C}$ Degradates ESI- Mode Analytes}

There were relatively small changes in recovery of degradates determined by ESI- mode after 28 days of storage (table 53). Most degradates were within the ASTM tolerable range after 28 days and above the practical limit of 70 percent recovery. A few degradates had recoveries less than 70 percent after 28 (4-(hydroxymethyl)pendimethalin) and 133 (acetochlor SA and cis-cyhalothric acid) days of storage, but the variability in each batch for these compounds (RSD $>30$ percent) might have obscured any trends in storage stability.

\section{Pesticide Stability Using Dechlorination Reagent (Ascorbic Acid) ESI+ Mode Analytes}

The addition of the dechlorination reagent ascorbic acid resulted in lower recoveries for about 18 percent of the ESI+ pesticides (table 54). Seven pesticides (indoxacarb, lactofen, 
propargite, pyridaben, tribufos, cis-permethrin, and transpermethrin) had recoveries lower than 70 percent on day 0 but exhibited no substantial change in concentration over time. This is likely the result of matrix suppression caused by the ascorbic acid. Another 11 pesticides (etoxazole, pymetrozine, diazinon, terbufos, chlorimuron-ethyl, chlorsulfuron, orthosulfamuron, prosulfuron, and the analytes with validation quality code "E" [bifenthrin, fenbutatin oxide, and flumiclorac-pentyl]) had large changes in recovery over time such that by day 28 recoveries were less than 70 percent. This indicates degradation of the pesticide was caused by the ascorbic acid because these analytes were stable in samples stored with no reagent. The ascorbic acid in general resulted in higher variability and slightly higher recoveries compared to reagent water with no ascorbic acid. For example mean recoveries of asulam were from 130 to 161 percent in ascorbic acid compared to 71 to 108 in reagent water alone.

The few degradates found in the samples spiked with pesticides only and stored with the dechlorination reagent ascorbic acid at $4{ }^{\circ} \mathrm{C}$ were the same ones as found in samples without acid and stored at $4{ }^{\circ} \mathrm{C}$. O-ethyl-O-methyl-S-propyl phosphorothioate was found at concentrations representing about 5 to 8 percent of the nominal concentration of the pesticide and remained at that level during storage. Fenamiphos sulfoxide, pyrimidinol (a diazinon degradate), and disulfoton sulfoxide stored with ascorbic acid increased from 5 percent to 12 to 38 percent over the 28-day storage, more than the samples stored in reagent water alone. In addition, phorate sulfoxide and terbufos sulfoxide increased to 7 and 6 percent over the 28-day storage in ascorbic acid.

\section{Pesticide Stability Using Dechlorination Reagent (Ascorbic Acid) ESI- Mode Analytes}

The addition of the dechlorination reagent ascorbic acid had no substantial effects on recoveries during storage in comparison to storage at $4{ }^{\circ} \mathrm{C}$ for pesticides determined by ESImode (table 55). Recovery of diflufenzopyr (60 percent) after 28 days was below the practical limit, but overall results were too variable to evaluate a definite trend. Recovery of flubendiamide ( 65 percent) after 28 days was also below the practical limit, but this was similar to stability results for samples stored with no reagent.

\section{Pesticide Degradate Stability Using Dechlorination Reagent (Ascorbic Acid) ESI+ Mode Analytes}

The addition of the dechlorination reagent ascorbic acid resulted in no change in recovery after 14 days and small changes in recovery after 28 days of storage for pesticide degradates determined in the ESI+ mode (table 56). Most analytes were within the ASTM limits after 28 days, and 75 of the 84 degradates were above the practical limit of 70 percent recovery. However, nine analytes (hydroxydiazinon, phorate oxon, terbufos oxon, 4-hydroxy-tert-fluometuron, 2-hydroxy-4-isopropylamino-6-amino-s-triazine, didealkylatrazine, hexazinone TP C, 1H-1,2,4-triazole, and diazinon oxon) were lower than
70 percent recovery after 28 days and some had large changes in recovery over time. This was clearly evident for the diazinon degradates: diazinon oxon, which decreased to 0 percent recovery after only 7 days of storage, and hydroxydiazinon, which decreased to 6 percent recovery after 28 days. Pyrimidinol initially increased from degradation of hydroxydiazinon or diazinon oxon to about 151 percent recovery and then later decreased. The other organophosphate degradates phorate oxon and terbufos oxon degraded during storage at $4{ }^{\circ} \mathrm{C}$ with no reagent, so the loss in this experiment is not due to the ascorbic acid. In addition, 2-hydroxy-6-ethylamino-4-amino-s-triazine recoveries changed from 177 percent on day 0 to 97 percent on day 28 , which might be an example of degradation obscured by the enhanced recovery.

\section{Pesticide Degradate Stability Using Dechlorination Reagent (Ascorbic Acid) ESI- Mode Analytes}

The addition of the dechlorination reagent ascorbic acid had no substantial effects on recoveries during storage for most pesticide degradates determined by ESI- mode (table 57). There was no substantial change in recovery over the 28-day storage time, although two analytes (cis-cyhalothric acid and hexazinone TP E) did not meet the 70 percent threshold for one and three of the time points, respectively. Variability was large on day 0 caused by one sample with low recoveries for most analytes.

\section{Pesticide Stability Using Antimicrobial Reagent (Citric Acid) ESI+ Mode Analytes}

The addition of the antimicrobial reagent citric acid to adjust the sample $\mathrm{pH}$ to 3.8 used in EPA method 527 resulted in changes in recovery for a small number of pesticides analyzed in the ESI+ mode (table 58). Many of the pesticides (76 of 106), mainly acetanilide, carbamate, fungicide, sulfonylurea, and triazine pesticides, had recoveries within the ASTM limits over the 28-day storage with citric acid. Only a few pesticide recoveries from these groups were less than the 70-percent-recovery practical limit after this time including the sulfonylureas chlorsulfuron, orthosulfamuron, prosulfuron, and the triazine cyanazine. However, many (15 of 43) miscellaneous and organophosphate pesticides had recoveries below the ASTM limit at three or more sampling times, probably due to matrix suppression caused by the acid. A few pesticides had large decreases in concentration over the storage time that was due to degradation caused by the acid, including etoxazole, pymetrozine, diazinon, orthosulfamuron, and prosulfuron. Bifenazate, terbufos and flumiclorac-pentyl also degraded over time in the acid, but these pesticide compounds also degraded in untreated water.

Few of the targeted degradates were found in the samples spiked with the pesticides only and stored with the citric acid. The organophosphate pesticide degradates O-ethyl-O-methylS-propyl phosphorothioate, fenamiphos sulfoxide, and terbufos sulfoxide were found at concentrations representing about 5 to 8 percent of the nominal concentration of the pesticide 
and remained at that level during storage. Pyrimidinol and disulfoton sulfoxide increased from 5 percent to 38 and 19 percent over the 28 -day storage, corresponding to decreases in the pesticide diazinon and disulfoton, similar to the samples stored in ascorbic acid.

\section{Pesticide Stability Using Antimicrobial Reagent (Citric Acid) ESI- Mode Analytes}

The addition of the antimicrobial reagent citric acid to adjust the sample pH to 3.8 used in EPA method 527 resulted in large changes in recovery for a number of pesticides analyzed in the ESI- mode (table 59). Many of the acids had high recoveries (mean recoveries from 106 to 517 percent), although there was no trend in recovery over time. The change in sample $\mathrm{pH}$ seems to have caused electrospray ionization enhancement in the MS/MS instrument for these acidic pesticides. Other effects of the citric acid were large variability for the sulfonylureas. Chlorsulfuron recoveries also were low (49 to 58 percent) and less than ASTM limits by day 14, similar to the samples treated with ascorbic acid and unlike those without reagent added. One degradate was found in the samples spiked with the pesticides only and stored with the citric acid. Diketonitrile isoxaflutole recoveries increased to 11 and 13 percent after 14 and 28 days of storage respectively, similar to samples stored without reagent added.

\section{Pesticide Degradate Stability Using Antimicrobial Reagent (Citric Acid) ESI+ Mode Analytes}

The addition of antimicrobial reagent citric acid resulted in no change in recovery after 14 days and small changes in recovery after 28 days of storage for most pesticide degradates determined in the ESI+ mode (table 60). Most analytes were within the ASTM limits after 28 days, and 73 of the 84 degradates were above the 70-percent-recovery practical limit. However, 11 degradates, mainly organophosphates and triazines (methomyl oxime, hydroxydiazinon, phorate oxon, terbufos oxon, phthalazinone, 4-hydroxy-tert-fluometuron, 2-hydroxy-6-ethylamino4-amino-s-triazine, deisopropylatrazine, didealkylatrazine, 1H-1,2,4-triazole, and diazinon oxon) were lower than 70 percent recovery after 28 days. Some degradates, including hydroxydiazinon, diazinon oxon, phorate sulfoxide, and terbufos sulfoxide, were degraded in the citric acid and had large decreases over the time of the study. The diazinon degradate pyrimidinol increased from degradation of hydroxydiazinon or diazinon oxon to 146 percent recovery by day 14 and then decreased.

\section{Pesticide Degradate Stability Using Antimicrobial Reagent (Citric Acid) ESI- Mode Analytes}

The addition of the antimicrobial reagent citric acid to adjust the sample $\mathrm{pH}$ to 3.8 used in EPA method 527 resulted in large changes in recovery for a number of pesticide degradates analyzed in the ESI- mode (table 61). Many of the acetanilide degradates had high recoveries (mean recoveries as much as 367 percent), although there was no trend in recovery over time. We assume this was the result of ionization enhancement. The variability of pesticide degradate recovery was also large, from 15 to 38 percent for many analytes. The hexazinone degradate hexazinone TP E had low recoveries ( 0 to 28 percent) at all times, most likely due to ionization suppression because there was no change in recovery over time.

\section{Summary and Recommendations Based on Stability Study Results}

The stability studies demonstrated that the largest number of the pesticide compounds (227 of 229) were stable in reagent water after 14 days of storage at $4{ }^{\circ} \mathrm{C}$, so that was selected as the practical holding time for routine sample processing. Upon arrival at the laboratory, samples are stored in a refrigerator and analyzed within 14 days of sample collection. The use of ascorbic acid as a dechlorination reagent resulted in lower recoveries of some analytes due to degradation and ion suppression, so use of this reagent to prevent reactions of pesticide compounds with free chlorine needs to be weighed against the negative effects of the reagent. The use of antimicrobial reagent citric acid to adjust the sample $\mathrm{pH}$ to about 4 also resulted in lower recoveries of some analytes, so it should not be used as a routine sample preservative. Storing the samples at $-10{ }^{\circ} \mathrm{C}$ for 28 to 133 days resulted in recoveries less than 70 percent for 10 of the 229 pesticide compounds and formation of four organophosphate degradates. If samples cannot be analyzed within the 14-day holding time, storing at $-10{ }^{\circ} \mathrm{C}$ would be a reasonable alternative for most of the pesticide compounds but should not be used for routine operation. If the samples are stored for longer than the holding time, laboratory reagent spikes should be prepared and stored with the samples to verify the results of the holding-time experiments described here, and appropriate data qualifiers should be assigned to sample results for analytes that exceed control limits.

\section{Problematic Compounds}

For a number of pesticide compounds (four in ESI+, six in ESI-), there was only one major product ion, so the qualifier MRM selected was precursor-to-precursor type (shown in bold in table 6). These pesticide compounds generally had higher MDLs (above $50 \mathrm{ng} / \mathrm{L}$ ) than other compounds, and identification in a sample requires careful evaluation by the analyst and comparison with calibration standards because these MRMs tend to have more background interferences.

Acetochlor SA and alachlor SA are not separated using the chromatography conditions of the DAI LC-MS/MS method, and they have the same quantifier $(314 \rightarrow 80)$ and qualifier ions $(314 \rightarrow 121$ ) MRMs (table 6). Additional unique but low abundance qualifier MRMs are used to identify these pesticide compounds, but the analyst needs to carefully compare ion ratios of samples with calibration standards for identification.

Siduron is the only pesticide compound that has cis- and trans-isomers separated using the chromatography conditions 
of the DAI LC-MS/MS method and require integration as one peak. This requires manual integration by the analyst because the automated integrator software identifies each peak as a separate compound. cis-Permethrin and trans-permethrin are also separated using the chromatography conditions of the DAI LC-MS/MS method, but these are integrated and reported as separate compounds.

Pymetrozine is an amine with pKa of 4.06 and therefore is appreciably ionic at low $\mathrm{pH}$. Consistent recoveries within data-quality objectives were obtained for pymetrozine in all three matrices (table 22) but were more variable $(\mathrm{RSD}=$ 40 percent) in the field-matrix-spike samples analyzed during the field study (table 28). This may be due to the inherent trade-off related to control of $\mathrm{pH}$ at 3-5 in samples with wider ranges of $\mathrm{pH}$. Analysts might need to qualify results based on variability in QC samples during an analytical run related to changes in sample $\mathrm{pH}$.

Asulam and chlorosulfonamide acid both have a chromatographic peak shape that changes, sometimes appearing as one peak, other times as two peaks. Asulam peak shape changes with concentration, whereas the peak shape of chlorosulfonamide acid is affected by matrix and age of the chromatographic column; both of these analytes require careful review and manual integration by the analyst.

Analytes that elute in the first few minutes of the chromatogram are prone to matrix-related electrospray ionization interference. For example, the triazine degradate didealkylatrazine (retention time $2.0 \mathrm{~min}$ ) had mean recoveries from 180 to 231 percent in all the matrices (tables 22 and 28). Other analytes that elute early and have interferences that cause problems with identification at low concentrations are 1H-1,2,4-triazole, 2-Hydroxy6-ethylamino-4-amino-s-triazine, and metribuzin DK.

\section{Summary and Conclusions}

A liquid chromatography-tandem mass spectrometry (LC-MS/MS) method was developed for determination of 229 pesticides compounds (113 pesticides and 116 pesticide degradates) in filtered water samples from stream and groundwater sites. The method involves direct injection of a $100-\mu \mathrm{L}$ sample onto the LC-MS/MS with filtration as the only sample preparation. Samples are analyzed with two injections, one in ESI positive mode and one in ESI negative mode, using dynamic MRM conditions, with two MRM transitions for each analyte. Isotope-labeled pesticide compounds are used as internal standards for quantitation to minimize effects of water-sample matrix components that cause ion suppression or enhancement. Information about the pesticide compounds included in the new method is provided in table 1, and a summary of results from selected validation studies is provided in table 62.

In the current method, DAI eliminates the need for sample extraction and lengthy sample preparation steps, which in turn eliminates analyte losses due to inefficient sample extractions or evaporation steps. Because the DAI LC-MS/MS method requires a relatively small sample volume $(100 \mu \mathrm{L})$, smaller sample volumes need to be collected and filtered than required for previous pesticide analytical methods. In addition, the specificity of the MS/MS technique allows use of disposable polypropylene sample collection equipment because plasticizer interferences in polypropylene that are a problem with single quadrupole MS instruments are not a problem with MS/MS. Together, these new requirements for sample processing offer substantial savings in sample collection and processing supplies, equipment, and time.

The pesticide compounds in the new method were considered high priority (tier 1) for method development and future monitoring studies in the USGS because of potential for occurrence and persistence in streams and groundwater, and toxicity to humans and aquatic organisms. The highpriority pesticide compounds included 78 new compounds not previously monitored in USGS analytical methods for determination of pesticides in water (based on their unique NWIS method code) as well as many pesticide compounds determined infrequently or in small research studies. In addition, 96 pesticide compounds are included in commonly used USGS analytical methods A unique feature of the new method is the relatively large number of pesticide degradates included (122 of 240), which provides the ability to more thoroughly study the fate of pesticides in the environment, as well as evaluate the toxicity and exposure effects.

The analytical method was first developed by selection and optimization of the MRM conditions for each pesticide compound. The MS/MS was operated in both positive and negative electrospray ionization modes for different groups of pesticide compounds. For most compounds in the ESI+ mode (171 of 185), the protonated molecular ion $[\mathrm{M}+\mathrm{H}]+$ was most abundant and was selected as precursor ion. For 10 pesticide compounds, the ammonia adducts $\left[\mathrm{M}+\mathrm{NH}_{4}\right]+$ were most abundant and were selected as precursor ion, but there were no differences in performance for these compounds compared to the compounds with protonated ion precursor (table 62). For most pesticide compounds in the ESI- mode (38 of 44), the deprotonated molecular ion [M-H]- was most abundant and was selected as precursor ion. For six pesticide compounds in the ESI- mode, there was only one major product ion, so the qualifier MRM selected was precursor-to-precursor type.

The method detection levels determined in reagent water range from 1 to $250 \mathrm{ng} / \mathrm{L}$, with a median of $3 \mathrm{ng} / \mathrm{L}$, for pesticide compounds determined in ESI+ mode, and from 2 to $250 \mathrm{ng} / \mathrm{L}$, with a median of $40 \mathrm{ng} / \mathrm{L}$, for pesticide compounds determined in the ESI- mode. These MDLs are comparable to or less than the MDLs of NWQL's existing analytical methods for pesticides.

Mean recoveries of most analytes in the ESI+ mode were within data-quality objectives of $100 \pm 30$ percent at all spike levels and matrices. The spiked sample recoveries in the three different matrices tested during a validation study were comparable to those in laboratory reagent water, indicating the absence of major matrix effects that caused ion suppression (or enhancement) of many of the pesticide compounds 
included in the method. A few pesticide compounds, such as 1H-1,2,4-triazole, 2-hydroxy-6-ethylamino-4-amino-s-triazine, methamidophos, didealkylatrazine, and chlorosulfonamide acid, elute in the first few min of the chromatographic run and have larger bias and variability because of interference from highly polar matrix components. Mean recoveries at medium and high concentrations $(100,500$, and 5,075 ng/L combined; $\mathrm{n}=10$ ) in reagent water were from 89 to 119 percent (median 104 percent), with RSD less than 22 percent for 169 analytes with MDLs above the spike level. More than half (96) of these analytes had recoveries between 95 and 105 percent, indicating low bias for many of the analytes in the DAI LC-MS/MS method. There was no obvious difference in recovery by type of pesticide (analytical method group). Mean recoveries in other matrices were similar, although there were some analytes with more bias in some matrices. The carbamates asulam and oxamyl oxime had low recoveries in all three environmental matrices but not in reagent water and thus were qualified with the permanent estimated remark (E). Recoveries at the lowest spike level $(10 \mathrm{ng} / \mathrm{L})$ had more bias and variability, especially when MDLs were near the spike level. Average recoveries of most analytes were within data-quality objectives of $100 \pm 30$ percent at spike levels above the calculated MDL. Recoveries of analytes in the ESI- mode were more variable in general than the ESI+ mode because many of the analytes have calculated MDLs higher than the lowest spike level.

Analysis of TPC standards demonstrate that the standards used in the DAI LC-MS/MS method provide comparable results to the existing methods for most analytes. Differences in environmental samples and field-matrix-spike recovery in comparison to samples analyzed by the different methods should be less than 20 percent based on calibration standard differences. Conversely, larger differences would be due to other factors such as matrix effects or differences in calibration but not the analytical standards. Detections of pesticide compounds in laboratory blanks were infrequent. There were 16 analytes with detections in the laboratory blanks in the ESI+ mode and two analytes with detections in the ESImode. There were four pesticide compounds (fenbutatin oxide, piperonyl butoxide, and pyrimidinol in the ESI+ mode and fipronil sulfone in the ESI- mode) with 3 to 6 detections in 17 laboratory blank samples (18 to 36 percent).

A comparison of the new DAI LC-MS/MS analytical method with two existing NWQL analytical methods (schedules 2033 and 2060) for determination of pesticides was conducted in collaboration with the NAWQA Program. Environmental samples, field-matrix spikes, and field blanks collected from a variety of NAWQA stream sites from June to September 2012, as well as routine laboratory QC samples, were analyzed by all three methods. The results provided a characterization of the performance of the new method during implementation in routine production, including bias and variability of analytes in schedule 2437 over a range of environmental matrices and concentrations and in comparison to schedules 2033 and 2060, potential for bias and contamination using the new field filtration procedure for schedule 2437 , and an opportunity to assess efficiency, costs, implementation, and logistical issues of schedule 2437 from both field and laboratory perspectives. Overall, the agreement in matrix spike recoveries, detection frequencies, and environmental concentrations was very good. At concentrations near the reporting levels, there were larger differences in detection frequencies and environmental concentrations. Significant differences in environmental concentrations were found between methods for some compounds. Recovery and other factors result in different environmental concentrations, and the differences vary by compound. These differences need to be understood and characterized prior to any data analysis when comparing data from different methods.

Agreement between different methods in number of detections found at concentrations near the LRL varies by pesticide compound. Different reporting levels in different methods result in fewer detections in one method. For example, LRLs are much lower in the LC-MS/MS method compared to the previous schedule 2060 for many pesticide compounds, so detection frequencies at low concentrations are larger with the LC-MS/MS method. Some pesticide compounds that have similar LRLs in reagent water have fewer detections in the LC-MS/MS method because matrix components can cause a decrease in one or both of the MRM signals or an increase in background noise.

Stability of the analytes added to laboratory reagent water and stored at different temperatures and in potential preservatives was measured in different studies. The stability studies demonstrated that the largest number of the pesticide compounds were stable in reagent water after 14 days of storage at $4{ }^{\circ} \mathrm{C}$, so that was selected as the practical holding time for routine sample processing. Upon arrival at the laboratory, samples are stored in a refrigerator and analyzed within 14 days of sample collection.

Recoveries of pesticides after 28 days of storage at $4{ }^{\circ} \mathrm{C}$ were relatively consistent. Only nine pesticides (etoxazole, indoxacarb, lactofen, disulfoton, phorate, terbufos, cis-permethrin, trans-permethrin, and flubendiamide) exceeded the practical limit of above 70 percent recovery after 28 days of storage, and all were within the practical limit after 14 days of storage. Similarly, 10 degradates exceeded the practical limit of 70 percent (phorate oxon, terbufos oxon, 4-hydroxy-tert-fluometuron, hydroxy mono demethyl fluometuron, hydroxyfluometuron, tebuthiuron TP 106, 2-hydroxy-4-isopropylamino-6-amino-s-triazine, 2-hydroxy-6-ethylamino-4-amino-s-triazine, didealkylatrazine, and hexazinone TP C), and all were within the practical limit after 14 days of storage except for phorate oxon and terbufos oxon. Few degradates were found in the samples spiked with pesticides only and stored at $4{ }^{\circ} \mathrm{C}$, indicating stability of most of the 44 pesticides that have one or more degradate measured in the ESI+ mode.

Changes in mean recovery of pesticides after 28 days of storage at $-10{ }^{\circ} \mathrm{C}$ were relatively low and are similar to the results of storage at $4{ }^{\circ} \mathrm{C}$. A few analytes (phorate, terbufos, phorate oxon, terbufos oxon, flumiclorac-pentyl, bifenazate) 
had higher recoveries after storage at $-10^{\circ} \mathrm{C}$ compared to $4{ }^{\circ} \mathrm{C}$. Storing the samples at $-10^{\circ} \mathrm{C}$ for 28 to 133 days resulted in recoveries of less than 70 percent for 10 of the 229 pesticide compounds and formation of four organophosphate degradates.

The addition of the dechlorination reagent ascorbic acid resulted in lower recoveries for some analytes, caused by both matrix suppression and degradation of the compound during storage. The ascorbic acid in general resulted in higher variability and slightly higher recoveries compared to reagent water with no ascorbic acid.

The addition of the antimicrobial reagent citric acid to adjust the sample $\mathrm{pH}$ to 3.8 used in EPA method 527 resulted in small changes in recovery for a number of pesticides analyzed in the ESI+ mode. A few pesticides had large decreases in concentration over the storage time that was due to degradation caused by the acid. The addition of the antimicrobial reagent citric acid to adjust the sample $\mathrm{pH}$ to 3.8 used in EPA method 527 resulted in large changes in recovery for a number of pesticide degradates analyzed in the ESI- mode, so it should not be used as a routine antimicrobial preservative.

\section{References Cited}

Alder, L., Greulich, K., Kempe, G., and Vieth, B., 2006, Residue analysis of 500 high priority pesticides - Better by GC-MS or LC-MS/MS?: Mass Spectrometry Reviews, v. 25 , no. 6 , p. $838-865$.

American Society for Testing and Materials, 1993, Standard practice for estimation of holding time for water samples containing organic and inorganic constituents, D4841-88, Annual Book of ASTM Standards, Section 11, Water and Environmental Technology, book 11.01 Water (II), sec. B: Philadelphia, Pa., American Society for Testing and Materials, p. 8-12.

Anisuzzaman, A.K.M., Storehalder, T., Williams, D.C., Ogg, N., Kilbourne, T.D., JohnSamuel, J., and Cottrell, C.E., 2008, Effect of alcohols on the stability of iprodione in solution: Journal of Agricultural and Food Chemistry, v. 56, no. 2, p. 502-506.

Backe, W.J., and Field, J.A., 2012, Is SPE necessary for environmental analysis? A quantitative comparison of matrix effects from large-volume injection and solid-phase extraction based methods: Environmental Science and Technology, v. 46, no. 12 , p. 6750-6758.

Bester, K., 2008, Quantification with HPLC-MS/MS for environmental issues - Quality assurance and quality assessment: Analytical and Bioanalytical Chemistry, v. 391, no. 1, p. $15-20$.

Childress, C.J., Foreman, W.T., Connor, B.F., and Maloney, T.J., 1999, New reporting procedures based on long-term method detection levels and some considerations for interpretations of water-quality data provided the U.S. Geological Survey National Water Quality Laboratory: U.S. Geological Survey Open-File Report 99-133, 19 p.

Díaz, L., Llorca-Pórcel, J., and Valor, I., 2008, Ultra trace determination of 31 pesticides in water samples by direct injection-rapid resolution liquid chromatography-electrospray tandem mass spectrometry: Analytica Chimica Acta, v. 624 , no. 1 , p. 90-96.

Eichelberger, J.W., Munch, J.W., and Shoemaker, J.A., 1995, Method 525.2. Determination of organic compounds in drinking water by liquid-solid extraction and capillary column gas chromatography/mass spectrometry-Methods for the determination of organic compounds in drinking water, suppl. III: U.S. Environmental Protection Agency, Office of Research and Development, National Exposure Research Laboratory EPA/600/R-95/131, 60 p.

European Commission, 2012, Method validation and quality control procedures for pesticide residues analysis in food and feed: European Commission Directorate General for Health and Consumers, SANCO/12495/2011, p. 41.

Fillâtre, Y., Rondeau, D., Jadas-Hécart, A., and Communal, P.Y., 2010, Advantages of the scheduled selected reaction monitoring algorithm in liquid chromatography/electrospray ionization tandem mass spectrometry multi-residue analysis of 242 pesticides - A comparative approach with classical selected reaction monitoring mode: Rapid Communications in Mass Spectrometry, v. 24, no. 16, p. 2453-2461.

Fuhrman, J.D., and Allan, J.M., 2003, Determination of acetanilide degradates in ground and surface waters by direct aqueous injection LC/MS/MS, in Ferrer, I., and Thurman, E.M., eds., Liquid Chromatography/Mass Spectrometry, Ms/Ms and Time-of-Flight Ms, book 850, sec. B: Washington, D.C., American Chemical Society ACS Symposium Series 850, p. 256-272.

Furey, A., Moriarty, M., Bane, V., Kinsella, B., and Lehane, M., 2013, Ion suppression-A critical review on causes, evaluation, prevention and applications: Talanta, v. 115, p. 104-122.

Furlong, E.T., Anderson, B.D., Werner, S.L., Soliven, P.P., Coffey, L.J., and Burkhardt, M.R., 2001, Methods of analysis by the U.S. Geological Survey National Water Quality Laboratory-Determination of pesticides in water by graphitized carbon-based solid-phase extraction and high-performance liquid chromatography/mass spectrometry: U.S. Geological Survey Water-Resources Investigations Report 01-4134, Method O-2060-01, 73 p.

Garbarino, J.R., Kanagy, L.K., and Cree, M.E., 2006, Determination of elements in natural-water, biota, sediment, and soil samples using collison/reaction cell inductively coupled plasma - mass spectrometry: U.S. Geological Survey Techniques and Methods, book 5, sec. B, chap. 1, p. 88. 
Greulich, K., and Alder, L., 2008, Fast multiresidue screening of 300 pesticides in water for human consumption by LC-MS/MS: Analytical and Bioanalytical Chemistry, v. 391, no. 1, p. 183-197.

Hahn, G.J., and Meeker, W.Q., 1991, Statistical intervals-A guide for practitioners: New York, Wiley, 392 p.

Helsel, D.R., and Hirsch, R.M., 1992, Statistical methods in water resources: New York, Elsevier, 522 p.

Helsel, D.R., and Hirsch, R.M., 2002, Statistical methods in water resources: U.S. Geological Survey Techniques of Water-Resources Investigations, book 4, chap. A3, available at http://water.usgs.gov/pubs/twri/twri4a3/.

Howard, P.H., ed., 1991, Handbook of environmental fate and exposure data for organic chemicals-Pesticides, v. III: Chelsea, Mich., Lewis Publishers, 684 p.

Huang, S.B., Mayer, T.J., and Yokley, R.A., 2008, Direct aqueous injection LC-ESI/MS/MS analysis of water for 11 chloro- and thiomethyltriazines and metolachlor and its ethanesulfonic and oxanilic acid degradates: Journal of Agricultural and Food Chemistry, v. 56, no. 8, p. 2595-2602.

Le Grand, R., Dulaurent, S., Gaulier, J.M., Saint-Marcoux, F., Moesch, C., and Lachâtre, G., 2012, Simultaneous determination of five synthetic pyrethroid metabolites in urine by liquid chromatography-tandem mass spectrometry-Application to 39 persons without known exposure to pyrethroids: Toxicology Letters, v. 210, no. 2, p. 248-253.

Lehotay, S.J., De Kok, A., Hiemstra, M., and Van Bodegraven, P., 2005, Validation of a fast and easy method for the determination of residues from 229 pesticides in fruits and vegetables using gas and liquid chromatography and mass spectrometric detection: Journal of AOAC International, v. 88 , no. 2 , p. 595-614.

Lehotay, S.J., Mastovska, K., Amirav, A., Fialkov, A.B., Martos, P.A., Kok, A., and Fernández-Alba, A.R., 2008, Identification and confirmation of chemical residues in food by chromatography-mass spectrometry and other techniques: TrAC Trends in Analytical Chemistry, v. 27, no. 11, p. 1070-1090.

Lovell, D.P., 2013, Biological importance and statistical significance: Journal of Agricultural and Food Chemistry, v. 61 , no. 35 , p. $8340-8348$.

Malcolm Pirnie Inc., 1999, Evaluation of the fate and transport of methanol in the environment: Malcolm Pirnie, Inc., accessed August 4, 2014, at http://www.methanol.org/Environment/ Resources/Environment/MP-Methanol-Fate.aspx.

Maloney, T.J., ed., 2005, Quality management system, U.S. Geological Survey National Water Quality Laboratory: U.S. Geological Survey Open-File Report 2005-1263, version 1.3 , chapters and appendixes variously paged.
Martin, J.D., and Eberle, M., 2011, Adjustment of pesticide concentrations for temporal changes in analytical recovery, 1992-2010: U.S. Geological Survey Data Series 630, 11 p.

Miller, J.C., and Miller, J.N., 1993, Statistics for analytical chemistry (3d ed.): New York, Ellis Horwood Limited, 233 p.

Murray, K.K., Boyd, R.K., Eberlin, M.N., John Langley, G., Li, L., and Naito, Y., 2013, Definitions of terms relating to mass spectrometry (IUPAC Recommendations 2013): Pure and Applied Chemistry, v. 85, no. 7, p. 1515-1609.

Norman, J.E., Kuivila, K.M., and Nowell, L.H., 2012, Prioritizing pesticide compounds for analytical methods development: U.S. Geological Survey Scientific Investigations Report 2012-5045, 74 p.

O’Boyle, N.M., 2012, Towards a universal SMILES representation-A standard method to generate canonical SMILES based on the InChI: Journal of Cheminformatics, v. 4, no. 22, p. 14.

Odetokun, M.S., Montesano, M.A., Weerasekera, G., Whitehead, R.D., Jr, Needham, L.L., and Barr, D.B., 2010, Quantification of dialkylphosphate metabolites of organophosphorus insecticides in human urine using 96-well plate sample preparation and high-performance liquid chromatography-electrospray ionization-tandem mass spectrometry: Journal of Chromatography B, v. 878, no. 27, p. 2567-2574.

Pepich, B.V., Prakash, B., Domino, M.M., Dattilio, T.A., Munch, D.J., and Price, E.K., 2005, Development of U.S. EPA method 527 for the analysis of selected pesticides and flame retardants in the UCMR survey: Environmental Science and Technology, v. 39, no. 13, p. 4996-5004.

Pozo, O.J., Sancho, J.V., Ibáñez, M., Hernández, F., and Niessen, W.M.A., 2006, Confirmation of organic micropollutants detected in environmental samples by liquid chromatography tandem mass spectrometry-Achievements and pitfalls: TrAC Trends in Analytical Chemistry, v. 25, no. 10, p. 1030-1042.

Radtke, D.B., Horowitz, A.J., and Sandstrom, M.W., 2004 with updates through 2009, Filtered samples: U.S. Geological Survey Techniques of Water-Resources Investigations, book 5, chap. A5, section 5.2, accessed October 14, 2009, at http://water.usgs.gov/owq/FieldManual/chapter5/html/ Ch5_contents.html.

Rowe, G.L., Gilliom, R.J., and Woodside, M.D., 2013, Tracking and forecasting the Nation's water quality-Priorities and strategies for 2013-2023: U.S. Geological Survey Fact Sheet 2013-3008, 6 p.

Sandstrom, M.W., Stroppel, M.E., Foreman, W.T., and Schroeder, M.P., 2001, Methods of analysis by the U.S. Geological Survey National Water Quality Laboratory-Determination of moderate-use pesticides in water by $\mathrm{C}-18$ solid-phase extraction and capillary-column gas chromatography/mass spectrometry: U.S. Geological Survey Water-Resources Investigations Report 01-4098, Method O-2002-01, 70 p. 
Sandstrom, M.W., and Lewis, J.A., 2009, Instructions for field use of spike solutions for organic-analyte samples: U.S. Geological Survey Techniques of Water-Resources Investigations, book 9, chap. A5, sec. 3.2, accessed October 14, 2009, at http://pubs.water.usgs.gov/twri9A5/.

SAS Institute Inc., 2010, Using JMP 9: Cary, N.C., SAS Institute Inc., $540 \mathrm{p}$.

Schlusener, M.P., and Bester, K., 2005, Determination of steroid hormones, hormone conjugates and macrolide antibiotics in influents and effluents of sewage treatment plants utilising high-performance liquid chromatography/tandem mass spectrometry with electrospray and atmospheric pressure chemical ionisation: Rapid Communications in Mass Spectrometry, v. 19 , no. 22, p. 3269-3278.

Shoemaker, J.A., and Bassett, M.V., 2005, Method 535. Measurement of chloroacetanilide and other acetamide herbicide degradates in drinking water by solid phase extraction and liquid chromatography/tandem mass spectrometry (LC/ MS/MS), ver. 1.1: Cincinnati, Ohio, U.S. Environmental Protection Agency, Office of Research and Development, National Exposure Research Laboratory, EPA Document 600/R-05/053, $61 \mathrm{p}$.

Shoemaker, J.A., 2009, Method 538. Determination of selected organic contaminants in drinking water by direct aqueous injection- liquid chromatography/tandem mass spectrometry (DAI-LC/MS/MS), ver. 1: Cincinnati, Ohio, U.S. Environmental Protection Agency, Office of Research and Development, National Exposure Research Laboratory, EPA Document 600/R-09/149, $40 \mathrm{p}$.

Shoemaker, J.A., 2011, Development and multi-laboratory verification of U.S. EPA method 538 for the analysis of drinking water contaminants by direct aqueous injectionLC/MS/MS: Analytical Methods, v. 3, no. 7, p. 1628-1636.

Sinclair, C.J., Boxall, A.B., Parsons, S.A., and Thomas, M.R., 2006, Prioritization of pesticide environmental transformation products in drinking water supplies: Environmental Science and Technology, v. 40, no. 23, p. 7283-7289.

Smith, G.A., Pepich, B.V., and Munch, D.J., 2011, Method 536. Determination of triazine pesticides and their degradates in drinking water by liquid chromatography electrospray ionization tandem mass spectrometry (LC/ESI-MS/ MS): Cincinnati, Ohio, U.S. Environmental Protection Agency, Office of Research and Development, National Exposure Research Laboratory, EPA 815-B-10-001, 36 p.

The Commission of the European Communities, 2002, 2002/657/EC - Commission Decision of 12 August 2002 implementing Council Directive 96/23/EC concerning the performance of analytical methods and the interpretation of results: Brussels, Official Journal of the European Communities, p. 36.
U.S. Geological Survey, 2006, Collection of water samples (ver. 2.0): U.S. Geological Survey Techniques of WaterResources Investigations, book 9, chap. A4, accessed August 4, 2014, at http://pubs.water.usgs.gov/twri9A4/.

U.S. Geological Survey, variously dated, National field manual for the collection of water-quality data: U.S. Geological Survey Techniques of Water-Resources Investigations, book 9, chaps. A1-A10, available at http://pubs.water.usgs.gov/ twri9A.

U.S. Geological Survey Office of Water Quality, 2004, Revised policy for the approval of U.S. Geological Survey Water-Quality Analytical Methods: Office Of Water Quality Technical Memorandum 04.01, accessed March 14, 2014, at http://water.usgs.gov/admin/memo/QW/qw04.01.html.

Weininger, D., 1988, SMILES, a chemical language and information system .1. Introduction to methodology and encoding rules: Journal of Chemical Information and Computer Sciences, v. 28, no. 1, p. 31-36.

Wilde, F.D., Sandstrom, M.W., and Skrobialski, S.C., 2014, Section 5.2.2.B. Syringe-filter procedure for processing samples for analysis of organic compounds by DAI LC-MS/MS: U.S. Geological Survey Techniques of WaterResources Investigations, book 9, chap. A5, accessed July 1, 2014, at http://water.usgs.gov/owq/FieldManual/chapter5/ html/Ch5_contents.html.

Winslow, S.D., Prakash, B., Domino, M.M., Pepich, B.V., and Munch, D.J., 2001, Considerations necessary in gathering occurrence data for selected unstable compounds in the USEPA Unregulated Contaminant Candidate List in USEPA Method 526: Environmental Science and Technology, v. 35, no. 9, p. 1851-1858.

Zaugg, S.D., Sandstrom, M.W., Smith, S.G., and Fehlberg, K.M., 1995, Methods of analysis by the U.S. Geological Survey National Water Quality Laboratory-Determination of pesticides in water by $\mathrm{C}-18$ solid-phase extraction and capillary-column gas chromatography/mass spectrometry with selected-ion monitoring: U.S. Geological Survey Open-File Report 95-181, Method O-1126-95, 49 p.

Zwiener, C., and Frimmel, F.H., 2004a, LC-MS analysis in the aquatic environment and in water treatment technology-A critical review. Part II. Applications for emerging contaminants and related pollutants, microorganisms and humic acids: Analytical and Bioanalytical Chemistry, v. 378, no. 4, p. 862-874.

Zwiener, C., and Frimmel, F.H., 2004b, LC-MS analysis in the aquatic environment and in water treatment-A critical review. Part I. Instrumentation and general aspects of analysis and detection: Analytical and Bioanalytical Chemistry, v. 378 , no. 4 , p. $851-861$. 


\section{Glossary}

analytical reference standard Samples of pure active ingredients or technical grade active ingredients of pesticides, and regulated metabolites, degradates, and related compounds, obtained from the EPA National Pesticide Standard Repository or other supplier.

bias Systematic error inherent in the method or measurement system. The error can be positive (for example, from contamination or ionization enhancement) or negative (from analyte loss or ionization suppression).

calibration (CAL) standard A solution prepared from the primary dilution standard solution and internal standard solution. The CAL solutions are used to calibrate the instrument response relative to analyte concentration.

concentrated standard solution A concentrated solution containing the individual analyte prepared in the laboratory using reference materials or purchased from a commercial vendor.

instrument blank (IBLNK) A sample of reagent water processed through the instrument analysis steps in the analytical process used to measure bias from the instrument analysis. Results for these samples analyzed by the DAI LC-MS/MS pesticide method are stored in the NWQL LIMS system as "instrument blank (IBLNK)" samples.

instrument detection level (IDL) standard A low-concentration calibration standard analyzed during the analytical run used to measure LC-MS/MS response during and at the end of the analytical batch. Results for these samples analyzed by the DAI LC-MS/ MS pesticide method are stored in the NWQL LIMS system as "instrument detection level (IDL)" samples.

internal standard (IS) solution A solution of isotope-labeled pesticides compounds used as internal standards for quantitation of method analytes. Internal standard recoveries are reported and used to monitor the method performance of a group of analytes in each sample, similar to surrogates.

internal standard (IS) An analyte added to the sample and used to measure the response of the pesticide compound relative to the internal standard - measured as chromatographic peak area for the quantitation MRM. Isotope-labeled pesticides or other chemicals similar to the pesticide compounds but not expected to be in the environmental sample are selected as internal standards.

laboratory reagent blank (PBLNK) A reagent-water sample processed through the sample preparation and analysis steps of the analytical process used to measure bias of the analytical method. Results for these samples analyzed by the DAI LC-MS/MS pesticide method are stored in the NWQL LIMS system as "preparation blank (PBLNK)" samples.

laboratory reagent spike (PSPK) A reagentwater sample spiked with the spiking solution and used to measure intra-laboratory bias and variability of the analytical method. The spiking solution is added in the laboratory prior to sample analysis. Results for these samples analyzed by the DAI LC-MS/MS pesticide method are stored in the NWQL LIMS system as "preparation spike (PSPK)" samples. The EPA uses the term "laboratory fortified blank" for this type of sample.

laboratory reporting level (LRL) The LRL is set at twice the MDL. The LRL is used to control false negative error - the risk of a false negative (not detecting an analyte when present) is less than 1 percent at the LRL (Childress and others, 1999).

matrix spike (MSPK) An environmental sample (stream or groundwater) spiked with the spiking solution and used to measure bias and variability due to sample matrix interferences. The spiking solution can be added at the collection site (field-matrix spike) or at the laboratory (laboratory matrix spike) and, if spiked at the collection site, is used to measure degradation during shipping and storage as well as matrix interferences. Results for these samples analyzed by the DAI LC-MS/MS pesticide method are stored in the NWQL LIMS system as "matrix-spike (MSPK)" samples.

method detection level (MDL) The MDL is the minimum concentration of an analyte that can be identified, measured, and reported with 99-percent confidence that the analyte concentration is greater than zero. At the MDL, the 
risk of a false positive (reporting a detection when no analyte is present) is predicted to be less than 1 percent. The MDL is determined from replicate analysis of low-concentration spike samples and, if done over an extended time period to obtain a more realistic measurement of the standard deviation, was termed the long-term MDL (Childress and others, 1999). The EPA uses the term "method detection limit."

mixed intermediate standard solution A solution containing a group of pesticide compounds based on pesticide use (herbicide, insecticide, fungicide), chemical class (acetanilide, triazine, acid), and whether it is a pesticide or degradate. The mixed solution is prepared from the individual concentrated standard solutions.

nominal concentration The amount of a pesticide compound that is expected to be present in a sample at the time the sample is prepared by spiking. Nominal is used as a synonym for "expected" in some contexts.

primary dilution standard solution A solution containing all the pesticide compounds prepared from all the mixed intermediate standard solutions.

reagent water Deionized water prepared by distillation, ion exchange, and filtration. The deionized water was then pumped through charcoal prefilters and a dual wavelength ultraviolet oxidizer lamp for removal of trace organic compounds to produce high-purity organic-free reagent water.

spiking solution A solution for preparing reagent spikes and matrix spikes. The spiking solution is prepared by dilution of the primary dilution standard solution.

surrogate An analyte added to the sample and used to measure the performance of the method in every sample. Isotope-labeled pesticides or other chemicals similar to the pesticide compounds but not expected to be in the environmental sample are selected as surrogates. The surrogate is chemically similar to the analyte so that losses in the analytical procedure are expected to be the same for both compounds.

tandem mass spectrometry or mass spectrometry/mass spectrometry (MS/MS) The acquisition and study of the spectra of the product ions or precursor ions of $\mathrm{m} / \mathrm{z}$ selected ions (Murray and others, 2013). third-party check (TPC) standards An analytical standard prepared from an independent source - different from that used to prepare the calibration and spike standards. The third-party check standard is used to verify that the calibration standards are accurate and have not changed as a result of degradation or errors in preparation. Results for these samples analyzed by the DAI LC-MS/MS pesticide method are stored in the NWQL LIMS system as "third-party check (TPC)" samples.

triple quadrupole mass spectrometer A tandem mass spectrometer comprising two transmission quadrupole mass spectrometers in series with a (nonselecting) radio frequencyonly quadrupole (or other multipole) between them to act as a collision cell (Murray and others, 2013).

variability Random error in independent measurements as the result of repeated application of the process or analytical method under specific conditions. 


\section{Appendix 1. Supporting Figures and Tables}

Supporting figure PDF files and supporting table Microsoft Excel files are available at http://dx.doi.org/10.3133/tm5B11.

\section{Supporting Figures}

Figure S1. The qualifier ion response ratio of pesticide compounds determined by liquid chromatography-tandem mass spectrometry (LC-MS/MS) in positive electrospray ionization (ESI+) mode fortified in different matrices (Santa Ana Prado Dam, Mississippi River, Well 1N/14W-8K1S) at 10, 100, 500, and 5,075 nanograms per liter (ng/L) and in reagent water (calibration [CAL] and continuing calibration verification [CCV] standards) at 1, 2.5, 5, $10,25,50,100,500,5,000$, and 10,000 ng/L.

Figure S2. The qualifier ion response ratio of pesticide compounds determined by liquid chromatography-tandem mass spectrometry (LC-MS/MS) in negative electrospray ionization (ESI-) mode fortified in different matrices (Santa Ana Prado Dam, Mississippi River, Well 1N/14W-8K1S) at 10, 100, 500, and 5,075 nanograms per liter (ng/L) and in reagent water (calibration [CAL] and continuing calibration verification [CCV] standards) at 1, 2.5, 5, $10,25,50,100,500,5,000$, and 10,000 ng/L.

Figure S3. Graphs of response factor (peak area/nominal concentration) and parameters of linear fit of response factor by nominal concentration for direct aqueous-injection liquid chromatography-tandem mass spectrometry (DAI LC-MS/MS) method positive electrospray ionization (ESI+) mode analytes.

Figure S4. Graphs of response factor (peak area/nominal concentration) and parameters of linear fit of response factor by nominal concentration for direct aqueous-injection liquid chromatography-tandem mass spectrometry (DAI LC-MS/MS) method negative electrospray ionization (ESI-) mode analytes.

Figure S5. Recovery of pesticide analytes determined by liquid chromatography-tandem mass spectrometry (LC-MS/MS) in positive electrospray ionization ( $\mathrm{ESI}+$ ) mode fortified at different concentrations $(10,100,500$, and 5,000 nanograms per liter [ng/L]) in four different matrices.

Figure S6. Recovery of pesticide analytes determined by liquid chromatography tandem mass spectrometry (LC-MS/MS) in negative electrospray ionization (ESI-) mode fortified at different concentrations $(10,100,500$, and 5,000 nanograms per liter [ng/L]) in four different matrices.
Figure S7. Recovery of pesticides determined by direct aqueous-injection liquid chromatography-tandem mass spectrometry (DAI LC-MS/MS) in field-matrix-spike samples by site in 200 field-matrix-spike samples that were prepared for the National Water Quality Assessment (NAWQA) Program field study from May to September 2012.

Figure S8. Pesticide compound recovery as a function of background environmental sample concentration determined by direct aqueous-injection liquid chromatography-tandem mass spectrometry (DAI LC-MS/MS) in 146 field-matrix-spike samples that were prepared for the National Water Quality Assessment (NAWQA) Program field study from May to September 2012.

Figure S9. Recovery of pesticides in reagent water after storage for as many as 28 days with different treatments (none, $4{ }^{\circ} \mathrm{C}$ [degrees Celsius]; none, $-10^{\circ} \mathrm{C}$; citric acid pH $3.8,4^{\circ} \mathrm{C}$; ascorbic acid, $4^{\circ} \mathrm{C}$ ) determined by direct aqueous-injection liquid chromatography-tandem mass spectrometry (DAI LC-MS/MS) in positive electrospray ionization (ESI+) mode.

Figure S10. Recovery of pesticide degradates in reagent water after storage for as many as 28 days with different treatments (none, $4{ }^{\circ} \mathrm{C}$ [degrees Celsius]; none, $-10^{\circ} \mathrm{C}$; citric acid pH 3.8, $4{ }^{\circ} \mathrm{C}$; ascorbic acid, $4^{\circ} \mathrm{C}$ ) determined by direct aqueous-injection liquid chromatography-tandem mass spectrometry (DAI LC-MS/ $\mathrm{MS}$ ) in positive electrospray ionization (ESI+) mode.

Figure S11. Pesticide degradates found in reagent water fortified with pesticides after storage for as many as 28 days with different treatments (none, $4^{\circ} \mathrm{C}$ [degrees Celsius]; none, $-10^{\circ} \mathrm{C}$; citric acid pH $3.8,4{ }^{\circ} \mathrm{C}$; ascorbic acid, $4^{\circ} \mathrm{C}$ ) determined by direct aqueous-injection liquid chromatography-tandem mass spectrometry (DAI LC-MS/MS) in positive electrospray ionization (ESI+) mode.

Figure S12. Recovery of pesticides in reagent water after storage for as many as 28 days with different treatments (none, $4{ }^{\circ} \mathrm{C}$ [degrees Celsius]; none, $-10^{\circ} \mathrm{C}$; citric acid pH $3.8,4^{\circ} \mathrm{C}$; ascorbic acid, $4^{\circ} \mathrm{C}$ ) determined by direct aqueous-injection liquid chromatography-tandem mass spectrometry (DAI LC-MS/MS) in negative electrospray ionization (ESI-) mode. 
Figure S13. Recovery of pesticide degradates in reagent water after storage for as many as 28 days with different treatments (none, $4^{\circ} \mathrm{C}$ [degrees Celsius]; none, $-10^{\circ} \mathrm{C}$; citric acid $\mathrm{pH} 3.8,4^{\circ} \mathrm{C}$; ascorbic acid, $4^{\circ} \mathrm{C}$ ) determined by direct aqueous-injection liquid chromatography-tandem mass spectrometry (DAI LC-MS/MS) in negative electrospray ionization (ESI-) mode.

Figure S14. Pesticide degradates found in reagent water fortified with pesticides after storage for as many as 28 days with different treatments (none, $4^{\circ} \mathrm{C}$ [degrees Celsius]; none, $-10^{\circ} \mathrm{C}$; citric acid $\mathrm{pH} 3.8,4^{\circ} \mathrm{C}$; ascorbic acid, $4^{\circ} \mathrm{C}$ ) determined by direct aqueous-injection liquid chromatography-tandem mass spectrometry (DAI LC-MS/MS) in negative electrospray ionization (ESI-) mode.

\section{Supporting Tables}

Table S1. Groups of pesticide compounds used to prepare intermediate standard solutions of pesticide compounds for direct aqueous-injection liquid chromatography-tandem mass spectrometry (DAI LC-MS/MS) method.

Table S2. Preparation of primary dilution standard solutions in methanol used to make calibration standards for direct aqueousinjection liquid chromatography-tandem mass spectrometry (DAI LC-MS/MS) method.

Table S3. Examples of National Water Information System (NWIS) qualifier codes used by analyst with direct aqueousinjection liquid chromatography-tandem mass spectrometry (DAI LC-MS/MS) method (laboratory schedule 2437) to qualify samples or analytes not meeting quality-control criteria.

Table S4. Summary of quality-control criteria for determination of pesticides by direct aqueous-injection liquid chromatographytandem mass spectrometry (DAI LC-MS/MS) (laboratory schedule 2437).

Table S5. Pesticide compounds identified as high priority for method development but not included in direct aqueous-injection liquid chromatography-tandem mass spectrometry (DAI LC-MS/ MS) method for various reasons.

Table S6. Summary of response factor (peak area/nominal concentration) and parameters of linear fit of response factor by nominal concentration for direct aqueous-injection liquid chromatography-tandem mass spectrometry (DAI LC-MS/MS) method positive electrospray ionization (ESI+) mode analytes.
Table S7. Summary of response factor (peak area/nominal concentration) and parameters of linear fit of response factor by nominal concentration for direct aqueous-injection liquid chromatography-tandem mass spectrometry (DAI LC-MS/MS) method negative electrospray ionization (ESI-) mode analytes.

Table S8. Recovery of pesticides determined by direct aqueousinjection liquid chromatography-tandem mass spectrometry (DAI LC-MS/MS) method (laboratory schedule 2437) in fieldmatrix-spike samples collected during National Water Quality Assessment (NAWQA) Program field comparison study from May to September 2012 excluded from data summaries because environmental concentration was greater than spike level.

Table S9. Summary of water quality parameters measured in streams when direct aqueous-injection liquid chromatographytandem mass spectrometry (DAI LC-MS/MS) method (schedule 2437) samples were collected during National Water Quality Assessment (NAWQA) Program field comparison study from May to September 2012.

Table S10. Summary of ancillary water quality parameters for each stream site when direct aqueous-injection liquid chromatography-tandem mass spectrometry (DAI LC-MS/MS) method (schedule 2437) samples were collected during National Water Quality Assessment (NAWQA) Program field comparison study from May to September 2012.

Table S11. Degradates found in reagent water samples spiked with pesticides spiked at 250 nanograms per liter (ng/L) and stored at $4{ }^{\circ} \mathrm{C}$ (degrees Celsius) for as many as 28 days and analyzed by direct aqueous-injection liquid chromatography-tandem mass spectrometry (DAI LC-MS/MS) in positive electrospray ionization $(\mathrm{ESI})$ and negative electrospray ionization (ESI-) modes.

Table S12. Degradates found in reagent water samples spiked with pesticides spiked at 250 nanograms per liter (ng/L) and stored at $-10^{\circ} \mathrm{C}$ (degrees Celsius) for as many as 133 days and analyzed by direct aqueous-injection liquid chromatography-tandem mass spectrometry (DAI LC-MS/MS) in positive electrospray ionization $(\mathrm{ESI}+)$ and negative electrospray ionization (ESI-) modes. 
Publishing support provided by:

Denver Publishing Service Center

For more information concerning this publication, contact: Chief, USGS National Water Quality Laboratory

Box 25585, Mail Stop 407

Denver, CO 80225-0585

(303) 236-2000

Or visit the National Water Quality Laboratory Web site at: http://nwql.usgs.gov/ 


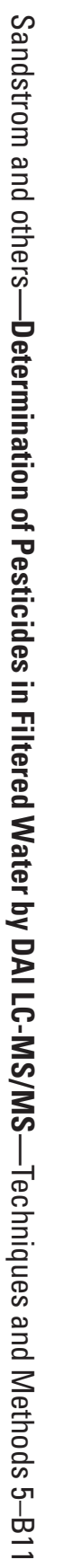

